\title{
A multi-adenylate cyclase regulator at the flagellar tip controls African trypanosome transmission
}

\section{Sabine Bachmaier}

Ludwig-Maximilians-Universität München (LMU) https://orcid.org/0000-0001-7416-8613

\section{Giacomo Giacomelli}

Ludwig-Maximilians-Universität München (LMU)

\section{Estéfania Calvo-Alvarez}

Institut Pasteur, Université de Paris

\section{Larissa Rezende Vieira}

Federal University of Rio de Janeiro https://orcid.org/0000-0003-1596-3953

\section{Jan Van Den Abbeele}

Institute of Tropical Medicine https://orcid.org/0000-0002-4533-4480

\section{Aris Aristodemou}

Ludwig-Maximilians-Universität München (LMU) https://orcid.org/0000-0002-3266-4499

\section{Esben Lorentzen}

Aarhus University

\section{Matthew Gould}

Ludwig-Maximilians-Universität München (LMU)

\section{Ana Brennand}

Ludwig-Maximilians-Universität München (LMU)

\section{Jean-Wiliam Dupuy}

University of Bordeaux https://orcid.org/0000-0002-2448-4797

\section{Ignasi Forné}

ludwig-Maximilians-University Munich (LMU)

\section{Axel imhof}

BioMedical center https://orcid.org/0000-0003-2993-8249

\section{Marc Bramkamp}

Kiel University https://orcid.org/0000-0002-7704-3266

\section{Didier Salmon}

Federal University of Rio de Janeiro

\section{Brice Rotureau}

Institut Pasteur

\section{Michael Boshart ( $\sim$ boshart@Imu.de)}

Ludwig-Maximilians-Universität München (LMU) https://orcid.org/0000-0002-5070-2663 


\section{Article}

\section{Keywords:}

Posted Date: January 25th, 2022

DOI: https://doi.org/10.21203/rs.3.rs-1216579/v1

License: (c) (i) This work is licensed under a Creative Commons Attribution 4.0 International License. Read Full License

Version of Record: A version of this preprint was published at Nature Communications on September 16th, 2022. See the published version at https://doi.org/10.1038/s41467-022-33108-z. 
1 A multi-adenylate cyclase regulator at the flagellar tip controls African 2 trypanosome transmission

3

4 Sabine Bachmaier ${ }^{1 *}$, Giacomo Giacomelli#, Estéfania Calvo-Alvarez ${ }^{3 \#}$, Larissa

5 Rezende Vieira ${ }^{4}$, Jan Van Den Abbeele ${ }^{5}$, Aris Aristodemou ${ }^{1}$, Esben Lorentzen ${ }^{6}$,

6 Matt K. Gould ${ }^{1}$, Ana Brennand ${ }^{1}$, Jean-Wiliam Dupuy ${ }^{7}$, Ignasi Forné ${ }^{8}$, Axel

$7 \quad$ Imhof $^{8}$, Marc Bramkamp 2 , Didier Salmon ${ }^{4}$, Brice Rotureau $^{3}$, Michael Boshart ${ }^{1 *}$

8

$9{ }^{1}$ Faculty of Biology, Genetics, Ludwig-Maximilians-University Munich (LMU),

1082152 Martinsried, Germany

112 Faculty of Biology, Microbiology, Ludwig-Maximilians-University Munich

12 (LMU), 82152 Martinsried and Institute for General Microbiology, Kiel

13 University, 24118 Kiel, Germany

$14{ }^{3}$ Institut Pasteur, Université de Paris, INSERM U1201, Trypanosome Cell

15 Biology Unit, Trypanosome Transmission Group, 75015 Paris, France

164 Institute of Medical Biochemistry Leopoldo de Meis, Centro de Ciências e

17 da Saúde, Federal University of Rio de Janeiro, Av. Brigadeiro Trompowski,

18 Rio de Janeiro 21941-590, Brazil

195 Trypanosoma Unit, Department of Biomedical Sciences, Institute of Tropical

20 Medicine Antwerp, 2000 Antwerp, Belgium

$21{ }^{6}$ Department of Molecular Biology and Genetics, Aarhus University, Gustav

22 Wieds Vej 10c, 8000 Aarhus C, Denmark

237 Univ. Bordeaux, Plateforme Protéome, 33000 Bordeaux, France 
$24{ }^{8}$ Biomedical Center, Ludwig-Maximilians-University Munich, 82152

25 Martinsried, Germany

26

$27 \quad$ \# authors equally contributed

$28{ }^{*}$ corresponding authors:

29 Sabine Bachmaier

30 sabine.bachmaier@Irz.uni-muenchen.de, Tel.: 49-89-2180-74600

$31 \quad$ Michael Boshart

32 boshart@Imu.de, Tel.: 49-89-2180-74600 


\section{Abstract}

34 Signaling from ciliary nanodomains controls developmental processes in

35 metazoans. Trypanosome transmission requires development and migration

36 in the tsetse vector alimentary tract. Flagellar cAMP signaling has been linked

37 to parasite social motility (SoMo) in vitro, yet uncovering control of directed

38 migration in fly organs is challenging. Here we show that the architecture of

39 an adenylate cyclase (AC) complex in the flagellar tip nanodomain is essential

40 for tsetse salivary gland (SG) colonization and SoMo. Cyclic AMP response

41 protein 3 (CARP3) binds and regulates multiple AC isoforms. CARP3 tip

42 localization depends on the scaffold FLAM8. Re-localization of CARP3 away

43 from the tip nanodomain is sufficient to abolish SoMo and fly SG colonization.

44 Since intrinsic development is normal in $\Delta$ carp3 and $\Delta$ flam8 mutant parasites,

45 AC complex-mediated tip signaling specifically controls parasite migration and

46 thereby transmission. Participation of several developmentally regulated

47 receptor-type $\mathrm{AC}$ isoforms may indicate the complexity of the in vivo signals

48 perceived. 
50 All cells perceive their environment and signal outside information to control

51 adaptive and developmental processes. For parasites, perception of the host

52 environment by physical and chemical cues is essential for survival and

53 transmission. Digenetic parasites face challenging rapid changes of biotic

54 environments between their reservoir and vector hosts ${ }^{1}$. Pathways processing

55 extracellular signals are not conserved in phylogenetically distant

56 kinetoplastids, including Trypanosoma ${ }^{2,3}$, and the connection of identified

57 signaling components remains to be solved. Trypanosomes shuttle between

58 mammalian and insect hosts and are well-studied due to medical and

59 economic impact as causative agents of neglected tropical diseases in

60 humans and animals ${ }^{4}$. A phenotype termed social motility (SoMo) ${ }^{5}$, a process

61 similar to bacterial swarming ${ }^{6}$, indicates intercellular communication or peer

62 signaling in populations of unicellular trypanosomes. On semisolid surfaces,

63 cultured procyclic insect forms of $T$. brucei migrate away from an initial

64 inoculation point by radial swarming 5 . Cyclic adenosine monophosphate

65 (cAMP) is key for control of SoMo ${ }^{7-9}$. Individual flagellar tip localized members

66 of a large multigene family of transmembrane receptor-type adenylyl cyclases

67 (ACs) are involved in the process ${ }^{7}$. Swarm-like collective motion of

68 trypanosomes in different tsetse tissues and organs has been observed ${ }^{10,11}$,

69 yet its relation to SoMo in vitro is unclear. A common mechanism for SoMo

70 and directed migration in the tsetse midgut has been suggested based on

71 correlated phenotypes upon deletion of PDEB19. 
72 Similar to the flagellum of mammalian sperm ${ }^{12}$, the trypanosome's single

73 flagellum represents a cAMP signaling compartment, as it displays a strong

74 enrichment of ACs and phosphodiesterases (PDEs), ubiquitous enzymes

75 producing or degrading cAMP, respectively. The trypanosome flagellar tip is a

76 site with particular importance for interaction with host tissue surfaces ${ }^{13}$ and

77 forms a distinct microdomain. This is defined by exclusive localization of a

78 subset of flagellar proteins ${ }^{14-17}$, including some members of the AC multigene

79 family ${ }^{18}$. The cAMP microdomain concept was first described by Buxton and

80 Brunton ${ }^{19}$ and is now well established in different systems ${ }^{20}$ including primary

81 cilia $^{21,22}$. The high spatiotemporal specificity of cAMP signaling within these

82 micro- or nanodomains depends on signaling cascades with sensor proteins,

83 transducers and effectors often arranged as multiprotein complexes, or

84 signalosomes, that can include scaffolding proteins such as AKAPs (A kinase

85 anchoring proteins). Localized PDEs limit the diffusion of the second

86 messenger and can generate cAMP concentration gradients across a cell,

87 while PDE inhibition has been shown to result in loss of cAMP

88 compartmentalization ${ }^{23}$. Within the very small cAMP nanodomains $(\sim 50-100$

$89 \mathrm{~nm}$ ), extremely high local cAMP concentrations are possible that can

90 specifically regulate even low affinity effectors located within the nanodomain.

91 Virtually nothing is known about cyclase regulation or downstream cAMP

92 signaling in kinetoplastids. These organisms lack known cyclase activators,

93 such as G protein-coupled receptors (GPCRs) and known cAMP effectors

94 including cAMP-dependent PKA ${ }^{24}$ or cyclic nucleotide-gated ion channels ${ }^{25}$.

95 The large family of receptor-type ACs with an extracellular N-terminal domain 
was suggested to provide the diversity for reception of multiple signals,

97 although no ligands of these ACs have been identified to date ${ }^{26}$. In

98 bloodstream form (BSF) parasites that infect the mammalian host,

99 disturbance of the intracellular cAMP concentration is critical for growth and

100 cytokinesis ${ }^{27-29}$. Exploiting this phenotype, a genome-wide RNAi screen for

101 cAMP resistance identified cAMP response proteins (CARPs) as candidates

102 for novel cAMP effectors or pathway modulators in T. brucei30. Among these,

103 CARP3 is a trypanosome-specific protein.

104 AC activity plays an important role in innate immunity subversion of

105 bloodstream stage trypanosomes early in infection ${ }^{31}$, The most abundant AC

106 involved is encoded by subtelomeric polycistronic transcription units driving

107 bloodstream form-specific variant surface glycoprotein expression (VSG

108 expression sites) ${ }^{32}$, hence named expression site-associated gene 4

109 (ESAG4). Trypanosomes shuttle between the bloodstream and tissues of a

110 mammalian host and the alimentary tract and salivary glands of a tsetse fly

111 undergoing a series of developmental transitions that result in defined

112 adapted stages $^{33}$. Cyclic AMP signaling was suggested to play a role in stage

113 development due to differential expression of transcripts encoding AC

114 isoforms in trypanosomes colonizing midgut, proventriculus or salivary glands,

115 respectively 34,35 . During their complex journey through the insect vector,

116 trypanosomes are in intimate contact with host tissue surfaces and have to

117 cope with several bottlenecks ${ }^{36}$.

118 Here, we provide direct evidence that a cAMP signalosome is essential for

119 trypanosome migration in the tsetse vector. Perturbation of the architecture of 
an $\mathrm{AC}$ complex at the flagellar tip is alone sufficient to abolish SoMo and

121 tsetse salivary gland colonization. The mutant parasites retain full intrinsic

122 developmental competence. The highly specific phenotype elicited by the 123 trypanosome-specific AC regulator CARP3 will stimulate further dissection of 124 novel mechanisms of cAMP signaling.

125

126

127

128

129

\section{Results}

\section{CARP3 is a flagellar tip protein essential for social motility and} colonization of tsetse fly salivary glands

We previously identified the cAMP response protein CARP3 in an RNAi screen for cAMP resistance in bloodstream forms (BSFs) of T. brucein. CARP3 is a protein with no sequence homology outside Trypanosoma and unknown biochemical functions, except $\mathrm{N}$-terminal myristoylation site(s) (http://lipid.biocuckoo.org/webserver.php). Its novel predicted structure (AlphaFold2 ${ }^{37}$ ) is shown in Extended Data Fig. 1a. As cAMP regulates social motility (SoMo) in procyclic forms (PCFs) ${ }^{8,38}$, we asked whether CARP3 did also control the parasite's colonization of the tsetse fly vector. A homozygous $C A R P 3$ deletion mutant, its endogenous single allele $C A R P 3$ rescue, as well as a tetracycline-inducible CARP3 RNAi cell line were generated in the fully differentiation-competent pleomorphic T. brucei strain AnTat 1.1 'Munich'39 and analyzed for in vitro culture phenotypes in SoMo, single cell motility, growth and differentiation. Deletion or depletion of $C A R P 3$ caused a complete block in SoMo (Fig. 1a,b). In contrast, single cell mean velocity in viscous medium, growth of freshly differentiated PCFs and BSFs and development of 
slender to stumpy BSFs and further to PCFs were all unaffected (Extended

147 Data Fig. 1b-g). The journey of trypanosomes in the tsetse fly vector proceeds

148 through additional well-defined developmental stages. The parasites migrate

149 from the insect's midgut via the cardia (proventriculus) to the salivary glands

150 (SGs) (Fig. 1c). Upon ingestion of stumpy BSFs by tsetse flies, carp3 KO cells

151 were unable to colonize the SGs, while showing high midgut infection rates

152 (Fig. 1d). The single-allele CARP3 rescue was sufficient to restore SG

153 infection rates to wild type levels. Indirect immunofluorescence analysis using

154 a CARP3-specific polyclonal antibody revealed localization of CARP3 to the

155 flagellar tip in PCFs (Fig. 1e, Extended Data Fig. 1h), consistent with

156 subcellular proteome studies ${ }^{16,17,40}$. CARP3 is concentrated at the tip of the

157 parental and daughter flagella (Fig. 1e) and it appears close to the flagellar

158 membrane as a horseshoe-shaped signal with a slight anterior to posterior

159 gradient (Extended Data Fig. 1h). No signal was detected in the carp3 KO cell

160 line (Fig. 1f). Surprisingly, in BSFs, CARP3 localized along the entire length of

161 the flagellum and at the posterior cell pole (Fig. 1g, Extended Data Fig. 1i)

162 with subcellular redistribution within the first hours of BSF to PCF

163 differentiation. This dynamic localization of CARP3 during parasite

164 development may correlate with life-cycle stage-specific functions. In PCFs,

165 immunofluorescence analysis also suggested colocalization of CARP3 and

166 Ty1-tagged adenylate cyclase isoforms ACP1 or ACP6 at the flagellar tip (Fig.

$1672 a)$. 
a

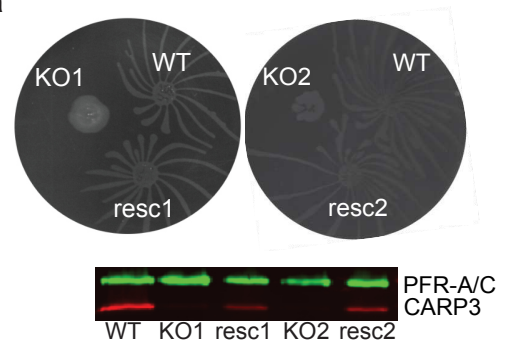

b

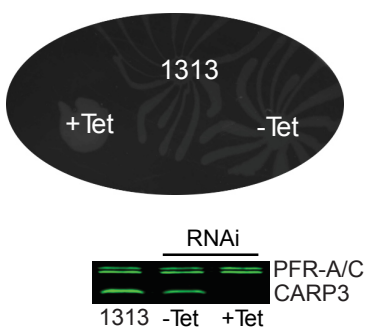

d

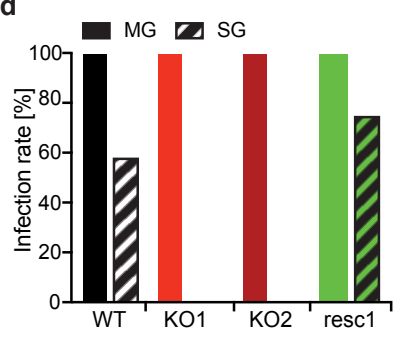

c

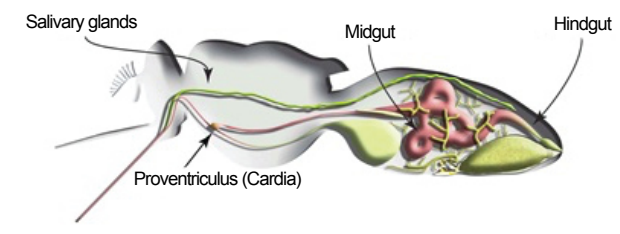

e

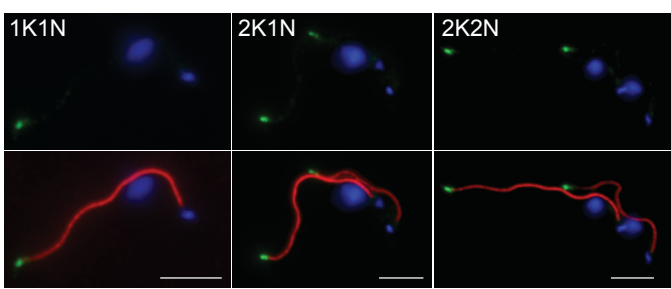

f

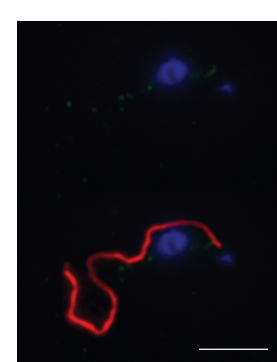

procyclic carp3 KO
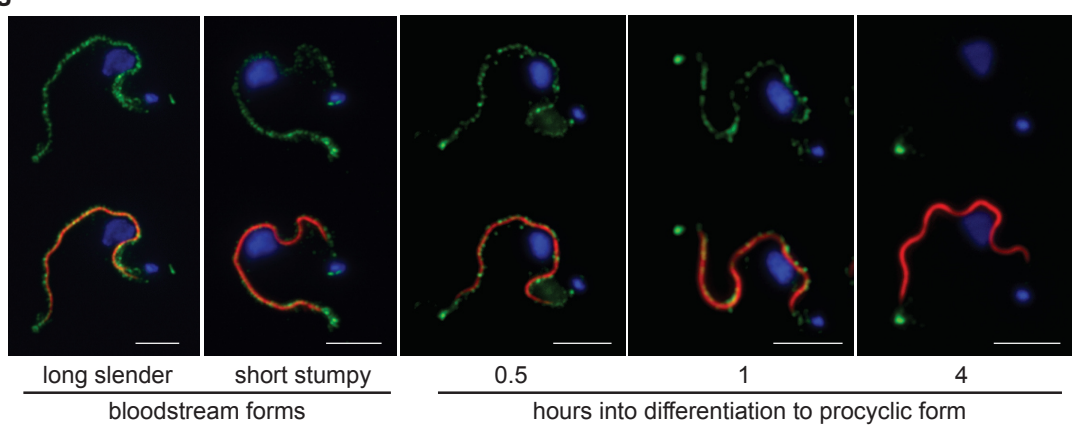

Fig. 1 CARP3 is a flagellar tip regulator of social motility (SoMo) and is essential for colonization of tsetse fly salivary glands.

171 a, SoMo assays of procyclic T. brucei AnTat 1.1 wild type (WT), carp3 knock out (KO, two independent clones $\mathrm{KO} 1, \mathrm{KO} 2$ ) or in situ CARP3 rescue (resc, two independent clones resc1, resc2). The Western blot for WT, KO1 and resc1 was probed with anti-CARP3 and anti-PFR-A/C (loading control). b, SoMo assay upon tetracycline (Tet)-inducible RNAi of CARP3 (-Tet / +Tet 24h) and the parental AnTat 1.11313 cell line. The Western blot shows CARP3 repression detected by antibodies as in (a). c, Illustration of the digestive system and the salivary glands of a tsetse fly (adapted from ${ }^{41}$ ). d, Infection rates of tsetse fly midgut (MG) or salivary glands (SG) with T. brucei AnTat 1.1 cell lines as in (a). Flies were dissected 34-36 days p.i., n (flies) = 48 (WT), 50 (KO1), 50 (KO2), 40 (resc1). 10 mM L-glutathione was included in the blood meal.

$180 \mathbf{e}, \mathbf{f}$, Indirect immunofluorescence analysis of CARP3 (green) in T. brucei AnTat 1.1 procyclic form WT (e) or carp3 KO (f). The upper panels show CARP3 (green) and the nuclear and mitochondrial DNA stained with DAPI (blue), the lower panels show an overlay with the axoneme (red; stained with the antibody mAB25). In (e) cells in different cell cycle stages are shown $(1 \mathrm{~K} 1 \mathrm{~N}, 2 \mathrm{~K} 1 \mathrm{~N}, 2 \mathrm{~K} 2 \mathrm{~N} ; \mathrm{K}$ kinetoplast,

184 N nucleus). g, Indirect immunofluorescence analysis of CARP3 (green) as in (e, f) during culture differentiation from bloodstream to procyclic forms. Scale bar in (e-g) $5 \mu \mathrm{m}$. 
Super-resolved spatial correlation of CARP3 with components of SoMo signaling

190 To precisely localize components of cAMP signaling involved in SoMo in the

191 tip microdomain, we used photo-activated localization microscopy (PALM) for 192 CARP3, ACP1 and phosphodiesterase PDEB1. One allele of CARP3 was C193 terminally fused in situ to photo-activatable mCherry (CARP3-PAmCherry). 194 The fusion protein was fully functional in SoMo, as deletion of the wild type 195 CARP3 allele in the transgenic line did not impair SoMo on agarose plates 196 (Fig. 2b). Quantitative colocalization analysis at single molecule resolution 197 was used to detect distinct subdomains at the flagellar tip (radius $\sim 300 \mathrm{~nm}$ ). 198 This confirmed close proximity of CARP3 to the flagellar tip membrane (Fig. 199 2c). In the same cell line, ACP1 was C-terminally fused in situ to 200 mNeonGreen (ACP1-mNG) (Extended Data Fig. 2a). ACP1-mNG localized to 201 the flagellar tip membrane (Fig. 2c and Extended Data Fig. 2b) as expected 202 from the presence of a transmembrane domain (TMD) and consistent with 203 previous reports ${ }^{17,18}$. The probability of colocalization between two 204 fluorescently tagged protein populations was determined by the coordinate205 based colocalization (CBC) analysis method of Malkusch, et al. ${ }^{42}$ (details see 206 methods). As positive control, we generated a cell line with one CARP3 allele 207 fused to PAmCherry and the other CARP3 allele fused to mNeonGreen 208 (Extended Data Fig. 2c). The CBC values' distribution for non-colocalizing 209 proteins (negative control) was instead obtained by simulating two 210 independent Poisson point patterns (Extended Data Fig. $2 f$ and 3, see 
211 methods for details). Comparison of the CBC values' distribution of CARP3-

212 PAmCherry and ACP1-mNG $(23.46 \%$ events with $\mathrm{CBC} \geq 0.5)$ with the 213 negative control ( $2.19 \%$ of events with $\mathrm{CBC} \geq 0.5$, see Supplementary Table

214 1) showed significant colocalization for CARP3-ACP1 $(p<0.05)$ that was in

215 the same range as the positive control (CARP3-PAmCherry-CARP3mNG;

$21622.22 \%$ events with $C B C \geq 0.5$, see Supplementary Table 1). CBC values

217 higher than 0.5 can be generally observed at the extreme tip of the flagellum 218 (Fig. 2d-f).

219 PDEB1 was also C-terminally fused to $\mathrm{mNeonGreen}$ and expressed in the

220 CARP3-PAmCherry line (Extended Data Fig. 2a). Live cell fluorescence

221 microscopy shows localization of PDEB1-mNG along the flagellum in a tip to

222 base decreasing gradient (Extended Data Fig. 2b). At single molecule

223 resolution, PDEB1-mNG does not localize to the far anterior tip of the

224 trypanosome flagellum, in agreement with previous localization to the

225 paraflagellar rod (Fig. 2 g) $)^{8,28,40}$. 
a

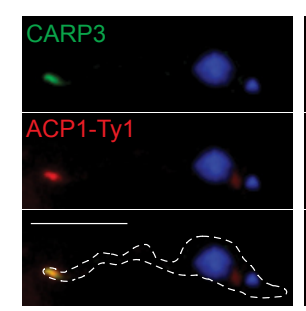

c

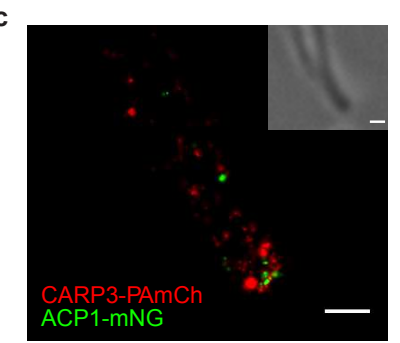

f

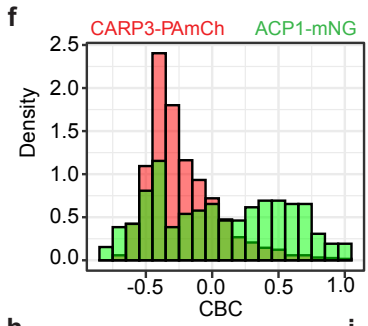

b
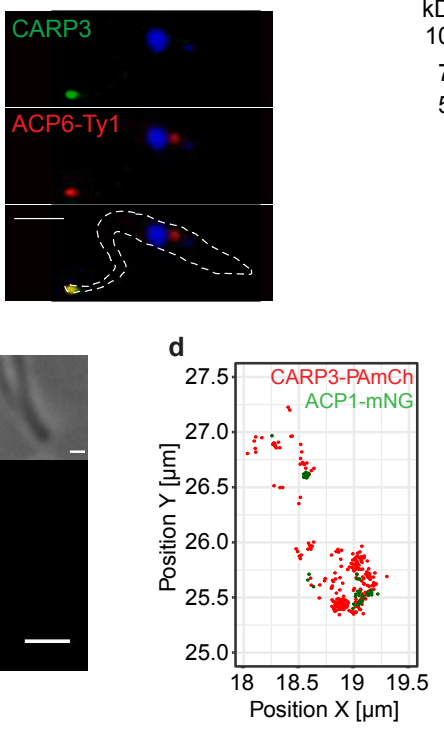

g

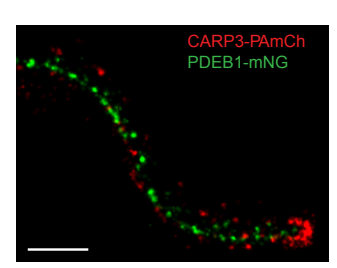

CARP3-PAmCh/
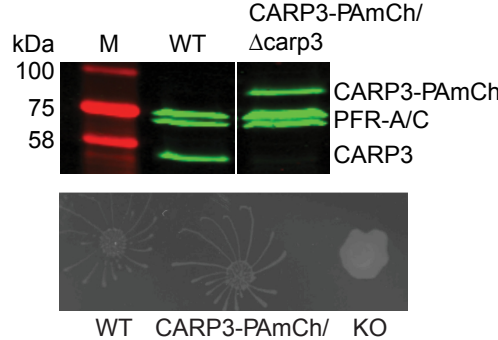

$\Delta$ carp3

e

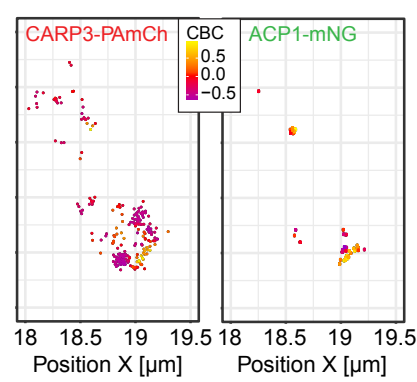

h
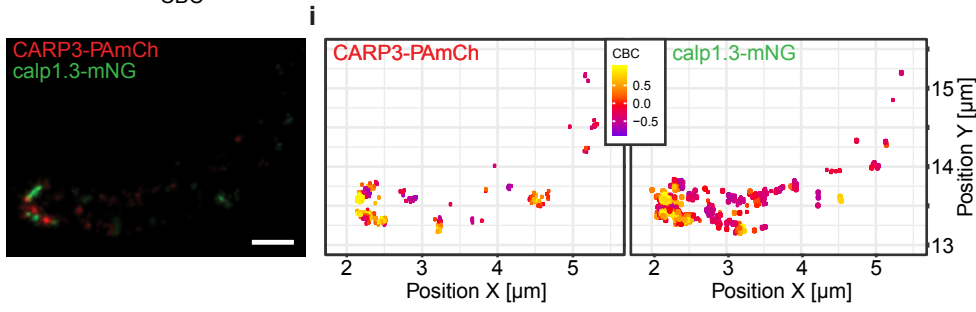

k
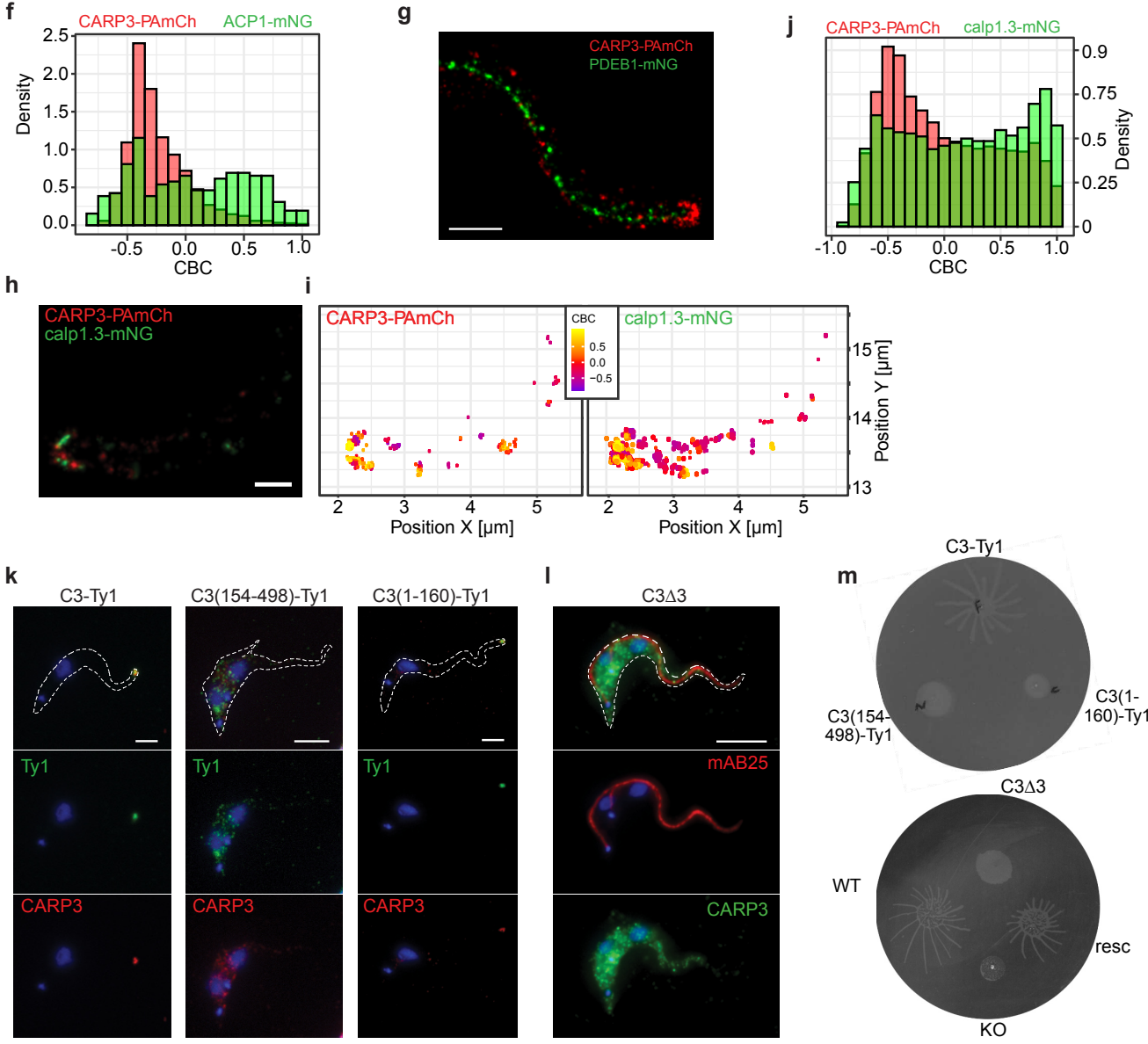

227 Fig. 2 Super-resolution topology in the flagellar tip nanodomain.

228 a, Indirect immunofluorescence analysis of CARP3 (green) and the adenylate cyclases ACP1 (left) or ACP6 (right), respectively, fused to a C-terminal Ty1 epitope tag (anti-Ty1, red) in procyclic T. brucei 29-

230 13. DNA was stained with DAPI (blue). Upper panels: CARP3 + DNA; middle panels: ACP1-Ty1 or ACP6-Ty1 + DNA; lower panels: merge of all three channels. Scale bars $5 \mu \mathrm{m}$. 

PAmCherry/Acarp3 cell line. Wild type (WT) and homozygous carp3 knock out (KO) cells were included as controls. The Western blot was probed with anti-CARP3 and anti-PFR-A/C (loading control). M: protein molecular weight marker.

236 c, PALM imaging of CARP3-PAmCherry (PAmCh, red) and ACP1-mNeonGreen (mNG, green) at the flagellar tip of procyclic T. brucei AnTat 1.1E. Corresponding phase contrast shown as inset. Scale bars

$2380.5 \mu \mathrm{m}$.

239 d, Single molecule localization from (c) is shown via centroids. CARP3-PAmCherry is displayed in red,

240 ACP1-mNeonGreen in green.

$241 \mathbf{e}, \mathbf{f}$, Colocalization analysis of CARP3-PAmCherry with ACP1-mNeonGreen. CARP3 (e, left) and ACP1

242 (e, right) localizations are color coded according to their respective Coordinate Based Colocalization

243 values $(\mathrm{CBC})$, where a higher $\mathrm{CBC}$ value signifies a higher likelihood of colocalization. $\mathrm{CBC}$ histograms

244 (f) of CARP3-PAmCherry (red) and ACP1-mNeonGreen (green) single molecule distributions. The

245 overlay of red and green bars is shown in dark green. All CBC values were calculated in a maximum

246 radius of $300 \mathrm{~nm}$ and radius intervals of $5 \mathrm{~nm}$. $\mathrm{n}=19$ flagella.

247 g, PALM imaging of CARP3-PAmCherry (red) and PDEB1-mNeonGreen (green) in the flagellum of 248 procyclic $T$. brucei AnTat 1.1E. Scale bar $0.5 \mu \mathrm{m}$.

$249 \mathbf{h}$-j, PALM imaging (h) and colocalization analysis (i, j) of CARP3-PAmCherry (red) and calpain 1.3$250 \mathrm{mNG}$ (green) at the flagellar tip of procyclic T. brucei AnTat 1.1E. CARP3 (i, left) and calpain 1.3 (i, 251 right) localizations are color coded according to their respective CBC values. Scale bar $0.5 \mu \mathrm{m}$.

252 Quantification in (j) shows CBC histograms of CARP3-PAmCherry (red) and calpain 1.3-mNG (green)

253 single-molecule distributions. The overlay of red and green bars is shown in dark green. $\mathrm{n}=15$ flagella.

$254 \mathbf{k}$, I, Indirect immunofluorescence analysis of CARP3 localization in procyclic T. brucei AnTat 1.1 upon 255 constitutive overexpression of CARP3-Ty1, CARP3(154-489)-Ty1 or CARP3(1-160)-Ty1 or in situ add256 back of $C A R P 3 \triangle 3$ in a carp3 knock out background. (k) Anti-CARP3 red, anti-Ty1 green, DAPI (DNA)

257 blue. (I) mAB25 (anti-TbSAXO) red, anti-CARP3 green. Scale bars $5 \mu \mathrm{m}$.

$258 \mathrm{~m}$, SoMo assay of cell lines as in $(\mathrm{k}, \mathrm{l})$ including WT and carp3 KO.

260 The $\mathrm{N}$-terminus of CARP3 is essential for flagellar tip membrane

\section{1 localization and SoMo}

262 In absence of a predicted transmembrane domain, membrane-proximal

263 localization of CARP3 suggests interaction with membrane(-associated)

264 proteins or (dual) acylation ${ }^{43,44}$. In order to position CARP3 relative to the

265 membrane at single-molecule resolution, we co-expressed CARP3-

266 PAmCherry with an mNeonGreen fusion of the previously characterized

267 flagellar tip protein calpain 1.3 (Fig. 2h; Extended Data Fig. 2a) that is 
associated with the membrane via $\mathrm{N}$-terminal dual acylation ${ }^{14}$. The CBC

values' distribution for the CARP3-PAmCherry / calpain 1.3-mNG combination

270 (Fig. 2i,j) showed significant colocalization with $33.9 \%$ of events with $\mathrm{CBC} \geq$

2710.5 , while the negative control remained at $2.19 \%(p<0.05)$ and the positive

272 control (CARP3-PAmCherry-CARP3mNG) at 22.22\% (Supplementary Table 1

273 and Extended Data Fig. 2e,f and 3). CARP3 was found in a chemical

274 proteomic survey of myristoylated proteins ${ }^{45}$ in addition to the sequence

275 prediction of myristoylation. The structural model of CARP3, computed using

276 AlphaFold ${ }^{37}$, shows a highly structured $\alpha$-helical domain predicted with high

277 confidence and interspaced by several long loops that are predicted with low

278 confidence and likely represent intrinsically disordered (ID) regions (Extended

279 Data Fig. 1a). To define the domain responsible for localization, deletion

280 mutants of CARP3 were expressed in a carp3 knock out background

281 (Extended Data Fig. 4a). Upon removal of the N-terminal 153 amino acids, C-

282 terminally Ty1-tagged CARP3 was no longer localized at the flagellar tip,

283 whereas expression of CARP3(1-160)-Ty1 was sufficient for flagellar tip

284 targeting (Fig. 2k). CARP3(1-160)-Ty1 seems to be unstable as its expression

285 level is too low for detection by Western blot (Extended Data Fig. 4a) but

286 sufficient for detection by IFA (Fig. 2k). Upon deletion of the three N-terminal

287 glycine residues (CARP3 $\triangle 3$ mutant), no CARP3 was detected at the flagellar

288 tip (Fig. 2l). The expression level of CARP3 $\Delta 3$ was comparable to that of full-

289 length CARP3 (Extended Data Fig. 4b). The deletion analysis strongly

290 supports an essential role of myristoylation as well as sufficiency of the N-

291 terminal structured domain for tip localization. Strikingly, all cell lines devoid of 
292 CARP3 at the flagellar tip were deficient in SoMo (Fig. 2m). We thus conclude

293 that the precise localization of CARP3 at the flagellar tip is critical for

294 trypanosome SoMo. The SoMo deficiency of the C-terminal CARP3 deletion

295 mutant (CARP3(1-160)-Ty1) is not informative due to very low expression

296 (Fig. 2m; Extended Data Fig. 4a).

297

298

FLAM8 is required for flagellar tip localization of CARP3

299 To define the extension of the CARP3 containing microdomain, colocalization

300 with FLAM8 (Flagellar Member 8) was tested. FLAM8 is a large cytoskeletal

301 flagellar tip protein proposed to localize at the plus end of the axonemal

302 microtubules in PCFs ${ }^{15}$. FLAM8 was C-terminally fused in situ to either YFP

303 (FLAM8-YFP) ${ }^{15}$ or mNeonGreen (FLAM8-mNG) and expressed in PCF

304 CARP3-mCherry or CARP3-PAmCherry cells, respectively (Extended Data

305 Fig. 2a,b). Whereas wide field fluorescence microscopy showed the expected

306 tip localization of both CARP3-mCherry and FLAM8-YFP (Fig. 3a), at single-

307 molecule resolution, distinct zones for CARP3-PAmCherry and FLAM8-mNG

308 were identified (Fig. 3c-f; Extended Data Fig. 5c). FLAM8 was found in the tip

309 interior compared to the membrane-proximal CARP3 in flagellar longitudinal

310 (Fig. 3c,d) and flagellar tip cross-sections (Fig. 3e,f). In a flagellar tip cross-

311 section with a radius of approximately $300 \mathrm{~nm}$, the two proteins were

312 characterized by a colocalization interface in a zone ranging between 150 and

$313200 \mathrm{~nm}$ from the center (Extended Data Fig. 5c). The CBC values' distribution

314 for the CARP3-PAmCherry / FLAM8-mNG combination shows $30.14 \%$ of

315 events with $\mathrm{CBC} \geq 0.5$ compared to $2.19 \%$ for the negative control (Fig. $3 g$, 
316 Extended Data Fig. 2f, Supplementary Table 1). This significant difference ( $p$

$317<0.05)$ is consistent with an association of a fraction of CARP3 with FLAM8 at

318 the proposed localization of FLAM8 at the plus end of the axonemal

319 microtubules ${ }^{15}$. Thus, separate pools of CARP3 seem to exist and FLAM8

320 might be involved in tip accumulation of CARP3. To test this hypothesis,

321 CARP3-mCherry was expressed in the $\Delta$ flam8 homozygous deletion

322 background ${ }^{46}$. Homogenous distributions of CARP3-mCherry (Fig. 3a) and

323 endogenous CARP3 (Fig. 3b) were seen along the length of the flagellum as

324 well as in the cytoplasm. In contrast, localization of FLAM8-YFP remained

325 unchanged in a carp3 knock out cell line (Fig. 3a). Given the essential role of

326 FLAM8 for the tip localization of CARP3, the SoMo deficiency of $\Delta$ flam8 cells

327 was not surprising (Fig. 3h). Controls verified that $\Delta$ flam 8 cells had single cell

328 motility and growth properties comparable to the parental cell line (Extended

329 Data Fig. 5a,b). Also, adding back a single FLAM8 allele fully restored wild

330 type SoMo (Fig. 3h). In summary, these data show that FLAM8 is essential for

331 tip localization of CARP3 and that delocalization of CARP3 is sufficient to

332 produce the SoMo-deficient phenotype. 

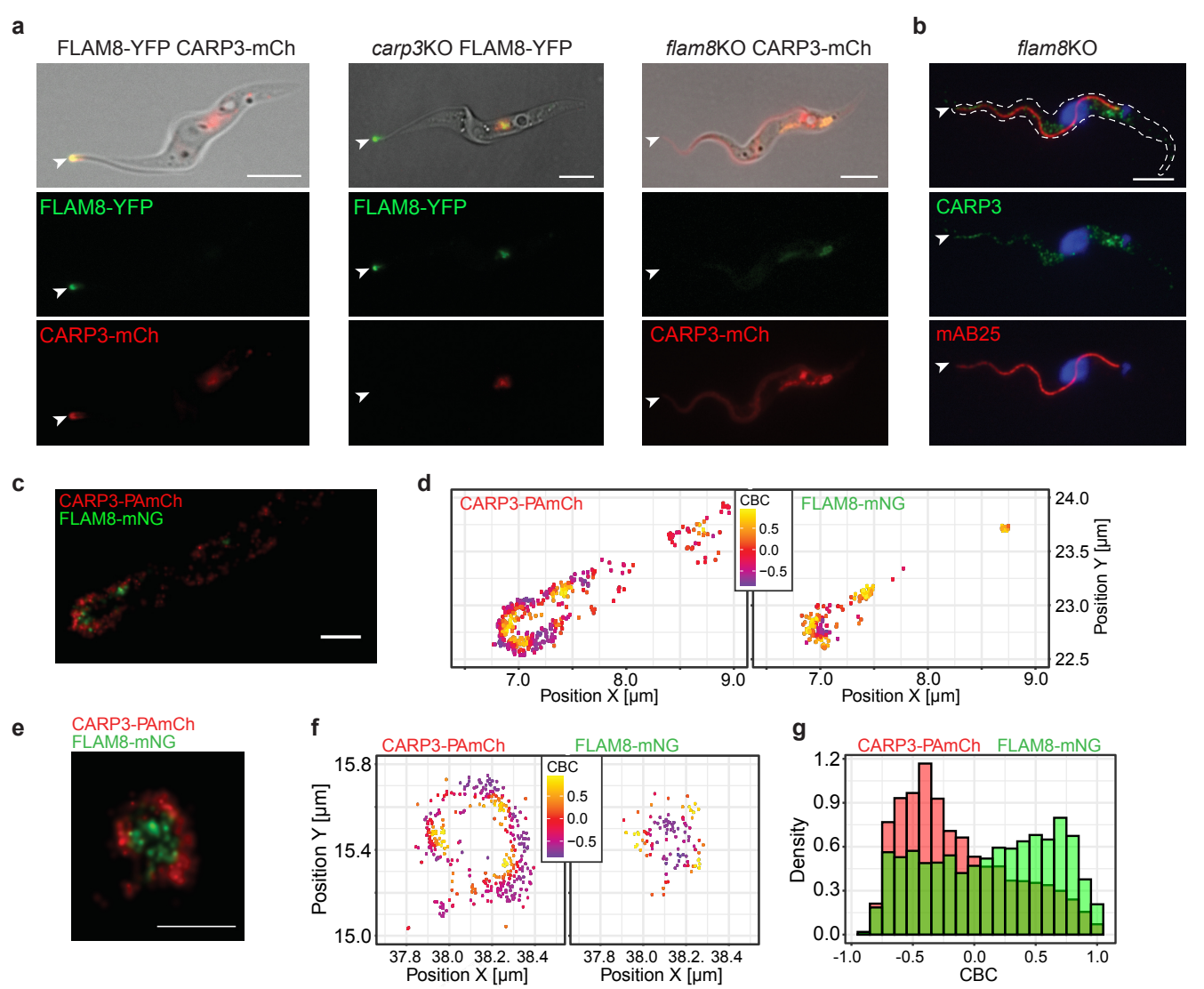

h

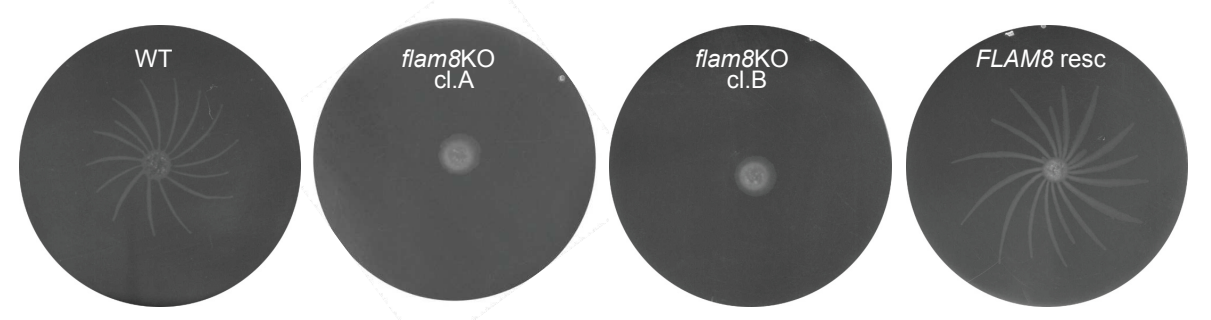

i

Cardia (Proventriculus) and foregut Salivary glands

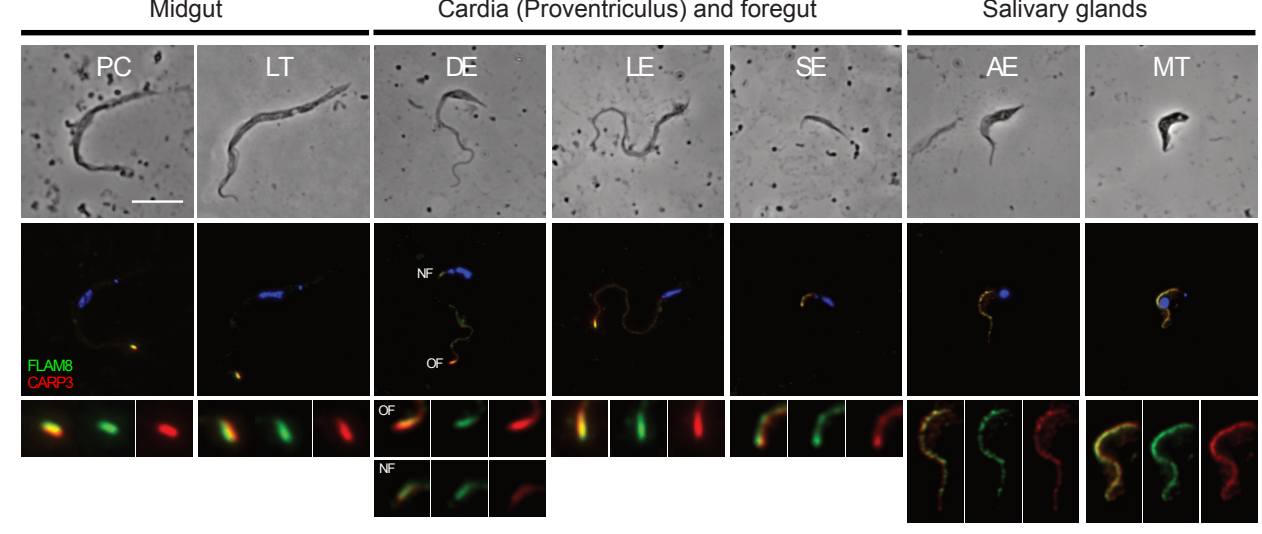

334 Fig. 3 FLAM8 is essential for flagellar tip localization of CARP3 and for SoMo.

335 a, Live cell fluorescence microscopy of procyclic T. brucei AnTat 1.1E expressing FLAM8-YFP (green) and CARP3-mCherry (CARP3-mCh, red) (left), AnTat 1.1 carp3 KO cells expressing FLAM8-YFP

337 (center) or AnTat 1.1E flam8 KO cells expressing CARP3-mCherry in (right). Top: merge of fluorescent 338 channels with DIC; middle: YFP; bottom: mCherry. 
b, Indirect immunofluorescence analysis of CARP3 (green) in procyclic T. brucei AnTat 1.1E flam8 KO cells. DNA was stained with DAPI (blue), the axoneme is labeled in red (detected by the antibody mAB25). Top: merge of fluorescent channels; middle: CARP3+DAPI; bottom: mAB25+DAPI. White arrowheads in (a) and (b) point towards flagellar tips; scale bars $5 \mu \mathrm{m}$.

343 c, d, PALM imaging (c) and colocalization analysis (d) of CARP3-PAmCherry (PAmCh) and FLAM8$\mathrm{mNeonGreen}(\mathrm{mNG})$ at the flagellar tip of procyclic T. brucei AnTat 1.1. CARP3 $(\mathrm{d}$, left) and FLAM8 $(\mathrm{d}$, right) localizations are color coded according to their respective $C B C$ values.

e, PALM imaging of CARP3-PAmCherry (PAmCh) and FLAM8-mNeonGreen (mNG) at a cross-section of the flagellar tip of procyclic T. brucei AnTat 1.1. Scale bars in (c) and (e) are $0.5 \mu \mathrm{m}$.

348 f, Colocalization analysis as in (d) for a flagellar tip cross section.

349 g, CBC histograms of CARP3-PAmCherry (red) and FLAM8-mNG (green) single molecule distributions 350 calculated from the data shown in panels (c-f). The overlay of red and green bars is shown in dark 351 green. $\mathrm{n}=27$ flagella.

$352 \mathrm{~h}$, SoMo assay of procyclic T. brucei AnTat 1.1E wild type (WT), flam8 KO (two subclones: A, B) or in 353 situ FLAM8 rescue (resc) cells expressing a fluorescent triple marker.

354 i, Distribution of CARP3 and FLAM8 during the parasite cycle in the tsetse fly. Immunofluorescence on methanol-fixed trypanosomes obtained from dissected tsetse tissues four weeks after infection. AntiCARP3 (red), anti-FLAM8 (green), DAPI (blue). Flagellum regions in white boxes on bright field are magnified in the bottom panel. Scale bar $5 \mu \mathrm{m}$. PC procyclic; LT long mesocyclic trypomastigote; DE dividing epimastigote; LE long epimastigote; SE short epimastigote; AE attached epimastigote; MT metacyclic; OF old flagellum; NF new flagellum.

361 Proximity of CARP3 and FLAM8 was observed in all developmental stages of

362 trypanosomes isolated from different tsetse fly alimentary tract compartments 363 and salivary glands (Fig. 3i). The flagellar tip colocalization found in midgut

364 (procyclic and long trypomastigotes) and cardiac stages (dividing and long 365 epimastigotes) was contrasting to the redistribution of both proteins along the 366 length of the flagellum in short epimastigotes in the cardia as well as in 367 attached epimastigotes and mammalian-infective metacyclic forms in the

368 tsetse salivary glands. The CARP3 localization pattern in salivary gland 369 stages was similar to that in bloodstream forms (Fig. 1g, Extended Data Fig. 370 1i). The dynamic localization of FLAM8 and CARP3 in the life cycle suggests 
371 a specific functional role at the flagellar tip in the parasite stages migrating in

372 the fly's alimentary tract.

373

374 Transmission-deficient trypanosomes are developmentally competent

375 We then tested the ability of $\Delta$ carp3 and $\Delta$ flam8 parasites to colonize tsetse

376 salivary glands and asked the crucial question whether the phenotypes were

377 due to migration or to an intrinsic developmental defect. Both mutants were

378 able to colonize the tsetse cardia, albeit with lower observed parasite

379 densities and at a reduced rate as compared to wild type cells (Fig. 4a-d).

380 Midgut infection rates were barely affected for $\Delta$ carp3 (Fig. 1d, Fig. 4a) and

381 reduced for $\Delta$ flam8 (Fig. 4c), respectively. The key finding is that not a single

382 mutant trypanosome reached the salivary glands in any of the 355 flies

383 dissected (100 flies infected with $\Delta$ carp3, Fig. 1d; 255 flies infected with

$384 \Delta$ flam8, Fig. 4c) in contrast to wild type parasites or rescued lines. Thus,

385 CARP3 and FLAM8 represent two genes that impact both SoMo and vector

386 colonization when deleted.

387 To check the impact of $C A R P 3$ deletion on the intrinsic developmental

388 competence of the parasite, we differentiated $\Delta$ carp3 parasites in culture

389 using inducible overexpression of the post-transcriptional regulator RBP6 48 .

390 RBP6 overexpression was induced in wild type and $\Delta$ carp3 parasites

391 (Extended Data Fig. 6), and followed by morphological analysis (Fig. 4e) and 392 stage-specific marker expression profiling (Extended Data Fig. 6d). Efficiency

393 and kinetics of differentiation from the procyclic form, via the epimastigote

394 morphotype, to the metacyclic stage was identical for wild type cells and 
$395 \Delta$ carp3 cells. Similarly, development of $\Delta$ flam8 parasites was analyzed in vivo 396 by imaging the parasites at different time points during their cyclical 397 development in the fly (Fig. 4f). Procyclic forms and long trypomastigotes 398 (mesocyclics) were found in the tsetse posterior midgut, and long 399 trypomastigotes and epimastigotes in the anterior midgut and cardia, showing 400 that these developmental stages are formed and viable in the absence of 401 FLAM8, hence in the absence of tip-localized CARP3. However, the number 402 of long trypomastigotes in the anterior midgut and cardia was reduced in $403 \Delta$ flam8 parasites as compared to WT, suggesting that long trypomastigote 404 parasites may be impaired in migration to or colonization of the anterior 405 midgut and/or cardia. These results exclude a defect in the intrinsic 406 developmental potential of the mutant trypanosomes and argue that the 407 strong salivary gland colonization phenotype is specifically due to a sensory 408 or signaling defect at the flagellar tip, possibly in long trypomastigotes, 409 impinging on forward migration in the tsetse fly digestive tract. 
a

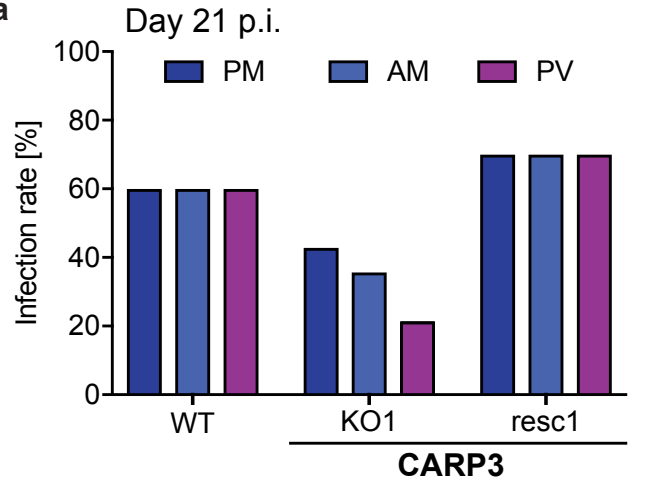

c

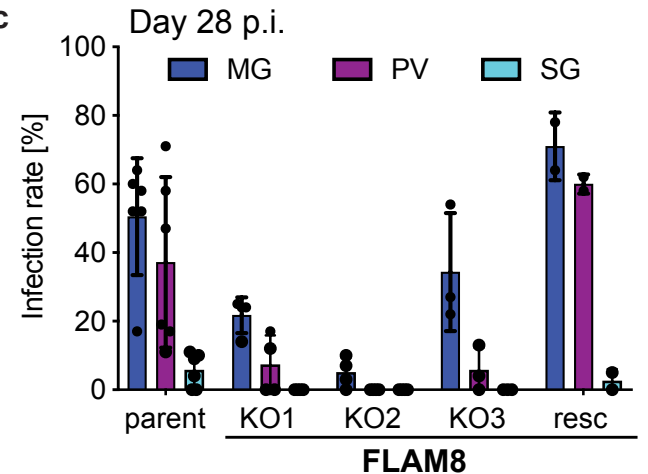

b

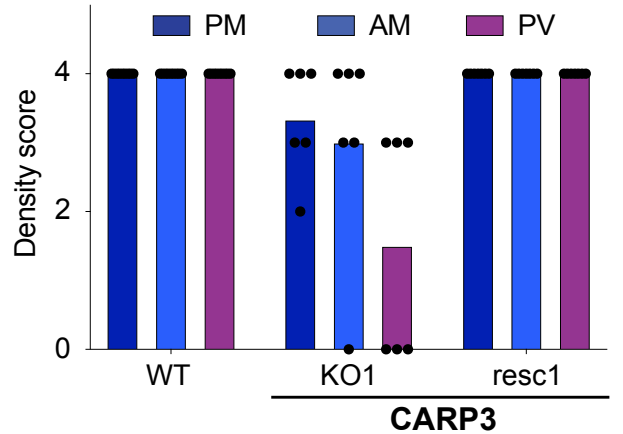

d

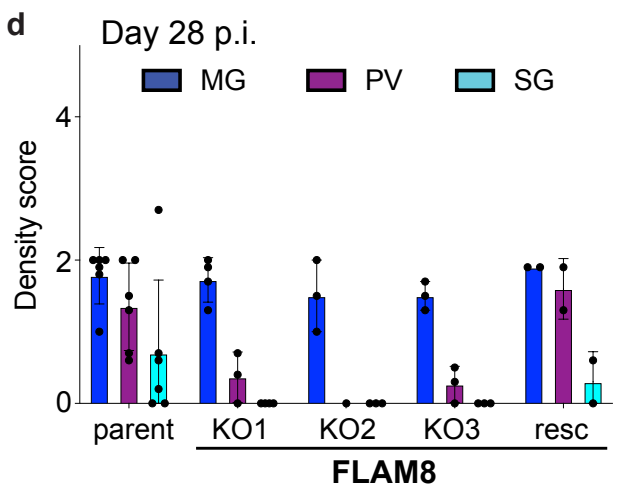

e

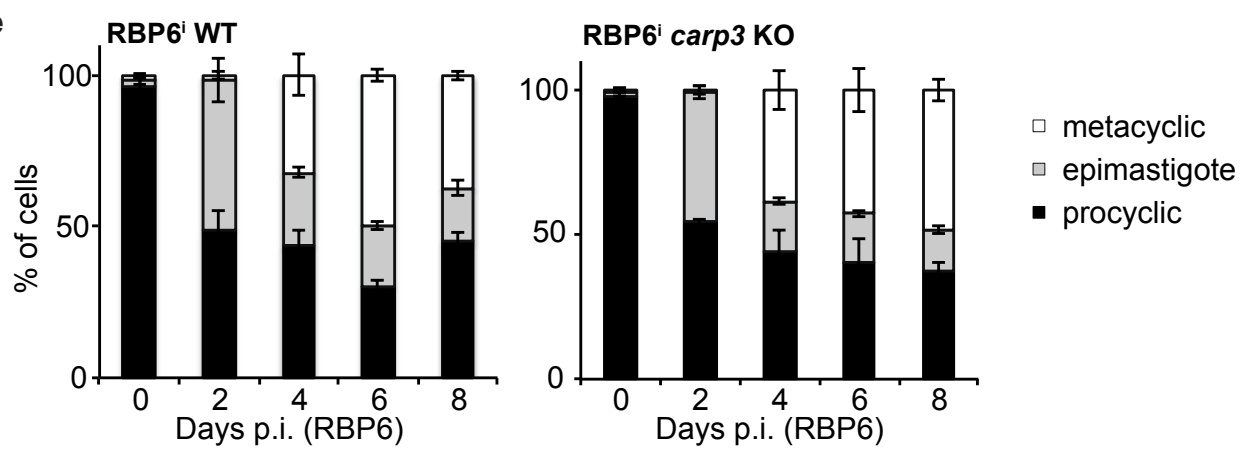

$\mathbf{f}$

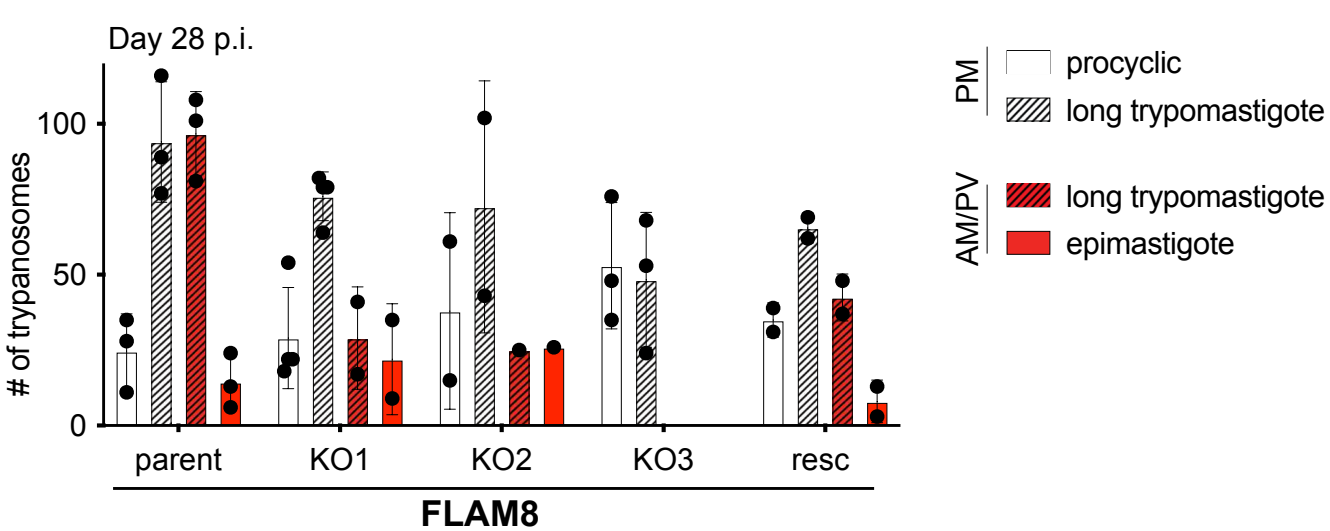

411 Fig. 4 Carp3 and flam8 KO parasites are developmentally competent but show reduced infection 412 of tsetse fly organs.

413 a, Infection rates and $\mathbf{b}$, parasite densities in posterior midgut (PM), anterior midgut (AM) or

414 proventriculus (PV or cardia) of tsetse flies infected with $T$. brucei AnTat 1.1 wild type (WT), carp3 knock

415 out (KO1) or in situ CARP3 rescue (resc1) cells (Institute of Tropical Medicine Antwerp tsetse fly 
colony). Flies were dissected 21 days p.i. n (flies) = 15 (WT), 14 (KO1), 10 (resc1). Parasite densities were scored only in flies with positive midgut infection. Density scoring (parasites per field): ‘4' > 1000, '3' 100-1000, '2' 10-100', '1' 1-10, '0' no parasites.

$\mathbf{c}$, Infection rates and d, parasite densities in midgut (MG), proventriculus (PV or cardia) or salivary glands (SG) of tsetse flies infected with $T$. brucei AnTat 1.1E 'Paris' (parent), flam8 KO (three subclones, KO1, KO2 and KO3) or in situ FLAM8 rescue cells (Institut Pasteur Paris tsetse fly colony). All cell lines express a red fluorescent triple marker ${ }^{47}$. Flies were dissected 28 days p.i.; $n$ (replicates) = 2-6, n (flies) = 122 (WT), 88 (KO1), 109 (KO2), 58 (KO3), 40 (resc). Density scoring as in (b).

e, Developmental progression upon RBP6 overexpression of carp3 KO trypanosomes compared to the parental RBP6 cell line. Procyclic, epimastigote and metacyclic forms were classified based on stagespecific characteristics including cell size and shape, position of the kinetoplast relative to the nucleus (visualized by staining with DAPI) and expression of stage-specific marker proteins (see Extended Data Fig. 6). $>100$ cells were analyzed at each time point and replicate. Mean $\pm S D$ of $n=3$. f, Quantification of trypanosomes in different developmental stages (procyclic, long mesocyclic trypomastigote, epimastigote) in posterior midgut (PM) or anterior midgut/proventriculus (AM/PV) of tsetse flies infected with $T$. brucei AnTat 1.1E 'Paris' (parent), flam8 KO (three subclones KO1, KO2, KO3) or FLAM8 rescue cell lines. Flies were dissected 28 days p.i. More than 50 trypanosomes were analyzed per fly (2-4 flies per group).

\section{CARP3 interacts with ACs}

As CARP3 was originally identified as a protein conferring cAMP resistance

437 and is also linked to cAMP via the SoMo phenotype, we tested direct binding of cAMP or association with a cAMP-binding partner. Cyclic AMP coupled to agarose beads pulled down the cAMP-specific phosphodiesterase PDEB1-

$440 \mathrm{mNG}$ (positive control) but not CARP3 from procyclic form lysates, indicating

441 that CARP3 is not likely a cAMP-binding protein (Supplementary Fig. 1a). Its

442 expression or localization in PCFs was also unaffected by even extreme

443 intracellular cAMP concentrations elicited by the PDE inhibitors CpdA or

$444 \mathrm{CpdB}^{27}$ (Supplementary Fig. 1b). Such conditions completely inhibited SoMo

445 (Supplementary Fig. 1c), as reported before ${ }^{8}$.

446 Potential CARP3 interaction partners were then identified by GFP-trap pull-

447 downs from $T$. brucei AnTat 1.1E CARP3-YFP of both BSF and PCF (Fig. 
5a,b and Extended Data Fig. 7a,b). Mass spectrometry analysis identified

CARP3 and 11 AC protein groups among the 14 protein groups significantly enriched ( $p \leq 0.05, \geq 10$-fold enrichment) in BSFs (Fig. 5a, Supplementary

451 Table 2a). In PCFs, CARP3 and 5 AC protein groups were among the 15

452 protein groups showing significant enrichment (Fig. 5b, Supplementary Table

453 2b). For independent confirmation, we selected ESAG4, an abundant, BSF-

454 specific AC, for which an antibody was available ${ }^{31}$. ESAG4 was pulled down

455 by CARP3-YFP (Extended Data Fig. 7c) as well as by native CARP3 (Fig. 5c).

456 For anti-CARP3-mediated pull-down, full-length ESAG4 was expressed as

457 GFP fusion protein (cell line DNi-3 from Salmon et al. ${ }^{31}$ ) enabling its detection

458 by in-gel fluorescence ${ }^{49}$. To identify the protein domain(s) of ESAG4 required

459 for interaction with CARP3, the intracellular C-terminal tail (107 amino acids,

460 ESAG4 $\triangle$ c107-GFP) following the AC catalytic domain of ESAG4 was

461 truncated (cell line DNi-2 ${ }^{31}$ ). This did not affect CARP3 interaction (Fig. 5c). In

462 contrast, deletion of the AC catalytic domain (cell line ESAG4DCAT-GFP)

463 abolished interaction. Both mutant proteins were expressed at a level

464 comparable to full-length ESAG4-GFP (Fig. 5c, input). Mass spectrometry

465 analysis of the reciprocal pull-down from ESAG4-GFP cells provided further

466 confirmation of ESAG4-CARP3 interaction (Extended Data Fig. 7d;

467 Supplementary Table 3). Besides CARP3, other AC isoforms were enriched

468 by ESAG4-GFP pull-down, indicating AC dimerization, as previously

469 reported ${ }^{18,26,31}$. Consistently, a highly confident homo-dimeric structure was

470 predicted using AlphaFold (Extended Data Fig. 8d). The physical interaction

471 between CARP3 and ACs was independently suggested by a CARP3 
472 proximity proteomics approach in BSFs (CARP3 BiolD; details see methods)

473 that revealed a significant enrichment $(3-115$-fold, FDR $\leq 0.05$, so $=2)$ of

474 several AC isoforms (Extended Data Fig. 7e; Supplementary Table 4). In this

475 proximity screen, we also found FLAM8 and calpain 1.3 enriched 125- and

476 83-fold, respectively; a result consistent with the high degree of colocalization

477 of FLAM8, calpain 1.3 and CARP3 in PCFs. Thus, a core complex seems to

478 be maintained at least throughout parts of the parasite life cycle. Physical

479 interaction of CARP3 with PCF-specific flagellar tip-localized AC isoforms was

480 directly shown by pull-down of ACP4-mNG and ACP5-mNG via CARP3

481 (Extended Data Fig. 7f,g). The CARP3-AC interaction was furthermore

482 supported by structural modeling using AlphaFold ${ }^{37}$ as well as the recently

483 released AlphaFold multimer ${ }^{50}$, which predicted models of CARP3 in complex

484 with each of seven different AC isoforms (ESAG4, GRESAG4.1, ACP1, 3, 4,

$4855,6)$. Superposition of all complex models shows a high degree of overall

486 structural similarity (root-mean-square-deviation of $0.8-1.4 \AA$ for $>90 \%$ of all

487 main-chain atoms) (Extended Data Fig. 8a-c). The interface consists of the

488 AC catalytic core and two N-terminal helices (residues 13-41) of CARP3, in

489 perfect agreement with our mutational analysis of the proteins. The interface

490 region has a very high degree of model confidence with a predicted local-

491 distance difference test (pLDDT) score>90 and a low predicted alignment

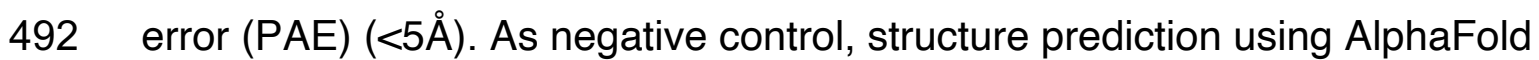

493 for a receptor-type AC of the distantly related kinetoplastid Bodo saltans and

494 T. brucei CARP3 resulted in a low confidence model with PAE $>20 \AA$ for

495 putative interface residues. 

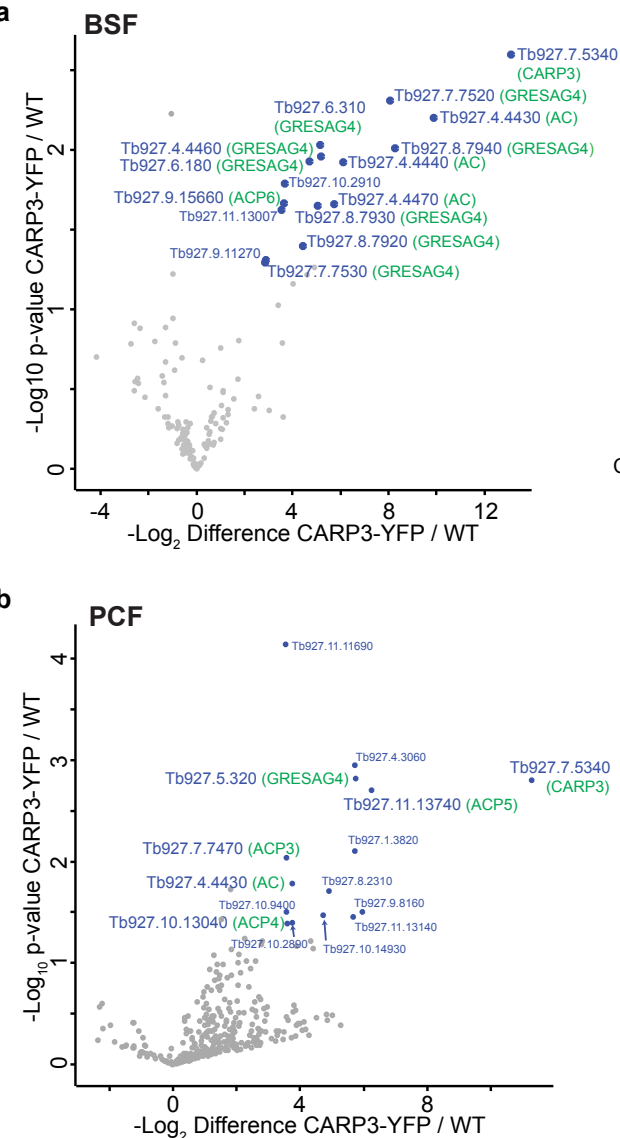
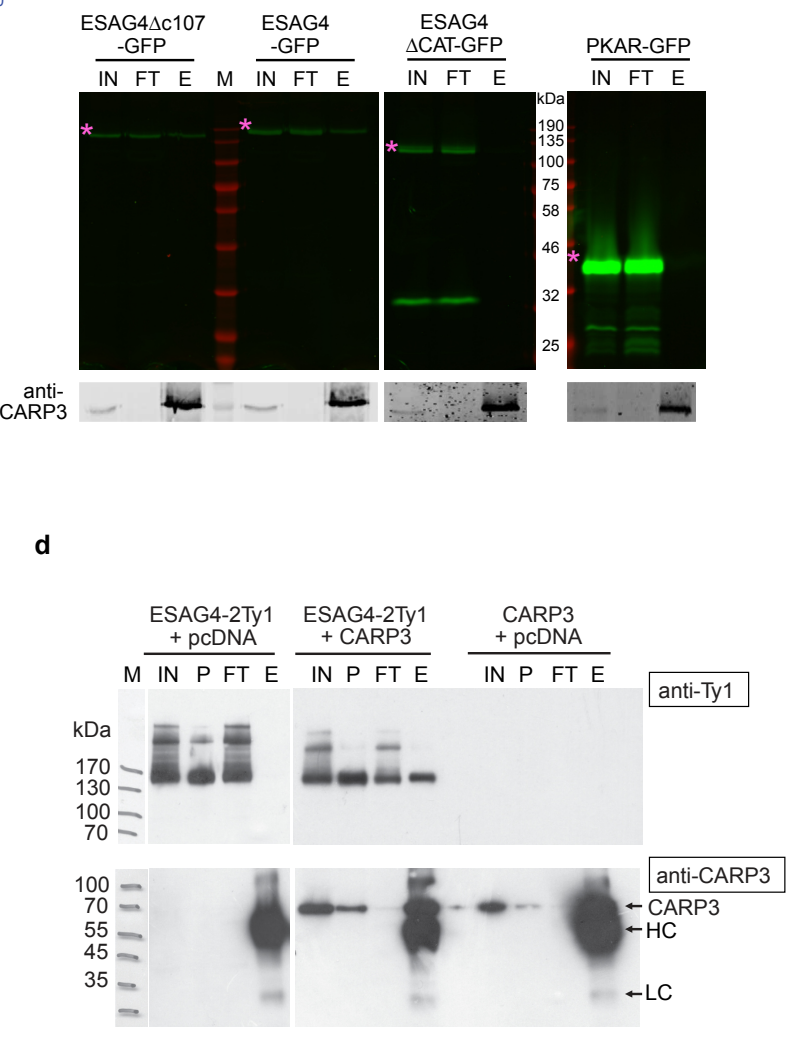

Fig. 5 CARP3 interacts with ACs.

a, b, Volcano plot representation of proteins identified upon GFP trap pull-down in T. brucei AnTat 1.1E CARP3-YFP cells compared to wild type (WT) in BSFs (a) or PCFs (b) ( $n=2$ replicate pull-downs each). Identified proteins are plotted according to $p$-value and fold change with significantly enriched proteins ( $p$-value $\leq 0.05$, fold change $\geq 10$ ) shown in blue with their TriTrypDB entries. The bait protein CARP3 and AC isoforms are indicated in green.

503 c, CARP3 immunoprecipitation (anti-CARP3 coupled to protein A beads) in BSF cell lines expressing 504 ESAG4-GFP, ESAG4 AC107-GFP, or ESAG4DCAT-GFP, respectively. Pull-down assays in a PKARGFP cell line served as negative control. Upper panels show detection of GFP by in-gel fluorescence, lower panels show Western blot detection of CARP3. The asterisk labels the fluorescent band corresponding to the respective GFP fusion protein. IN input; FT flow-through; E elution (10x load of input or flow-through); M protein molecular weight marker. HC anti-CARP3 heavy chain d, CARP3 immunoprecipitation (anti-CARP3 coupled to protein A beads) from soluble fractions of HEK293 cells expressing CARP3 or ESAG4-2Ty1 or both. Equal amounts of total DNA were transfected in all conditions (ratio of transfected plasmids 1:1). The upper Western blot was probed with anti-Ty1, the lower with anti-CARP3. IN soluble input; P insoluble pellet; FT flow-through; E elution; M protein molecular weight marker; HC anti-CARP3 heavy chain; LC anti-CARP3 light chain. 
517 We expected the CARP3-AC interaction to modulate AC catalytic activity in

518 the complex. CARP3 and ESAG4 were co-expressed in mammalian HEK293

519 cAMP reporter cells that express luciferase under control of a cAMP response

520 element (CRE). The CARP3-AC interaction was maintained in the

521 heterologous system as shown by pull-down (Fig. $5 d$ ). This provided

522 additional evidence for direct physical interaction independent of the parasite

523 cellular environment. Surprisingly, the amount of cAMP produced by

524 recombinantly expressed ESAG4 and normalized to the ESAG4 level was

525 independent of the amount of co-expressed CARP3 in the heterologous HEK

526 cell system (Extended Data Fig. 7h). In contrast, CARP3 knock down resulted

527 in strongly reduced cAMP production (swell dialysis AC assay) (Fig. 6a) and a

528 decrease in ESAG4 levels over $24 \mathrm{~h}$ in BSFs (Fig. 6b). Quantitative

529 proteomics confirmed a 13-fold and 2 -4-fold decrease of CARP3 and five AC

530 groups, respectively (Fig. 6c, Supplementary Table 5). No other significant

531 proteome changes were detected $(p \leq 0.05)$. The proteomic data show that

532 CARP3 is a multi-AC regulator (Supplementary Tables 5 and 6). An

533 approximate 3-fold decrease in the expression of ACP6 upon CARP3 knock

534 down determined by Western blotting in procyclic cells (Fig. 6d) confirmed the

535 regulation in PCF and suggested that the SoMo and fly colonization

536 phenotypes elicited by CARP3 are likely due to changes in AC abundance.

537 Taken together, these results show that CARP3 is a novel modulator of

538 flagellar tip cAMP levels by stabilizing AC abundance in this cAMP

539 microdomain. 
a
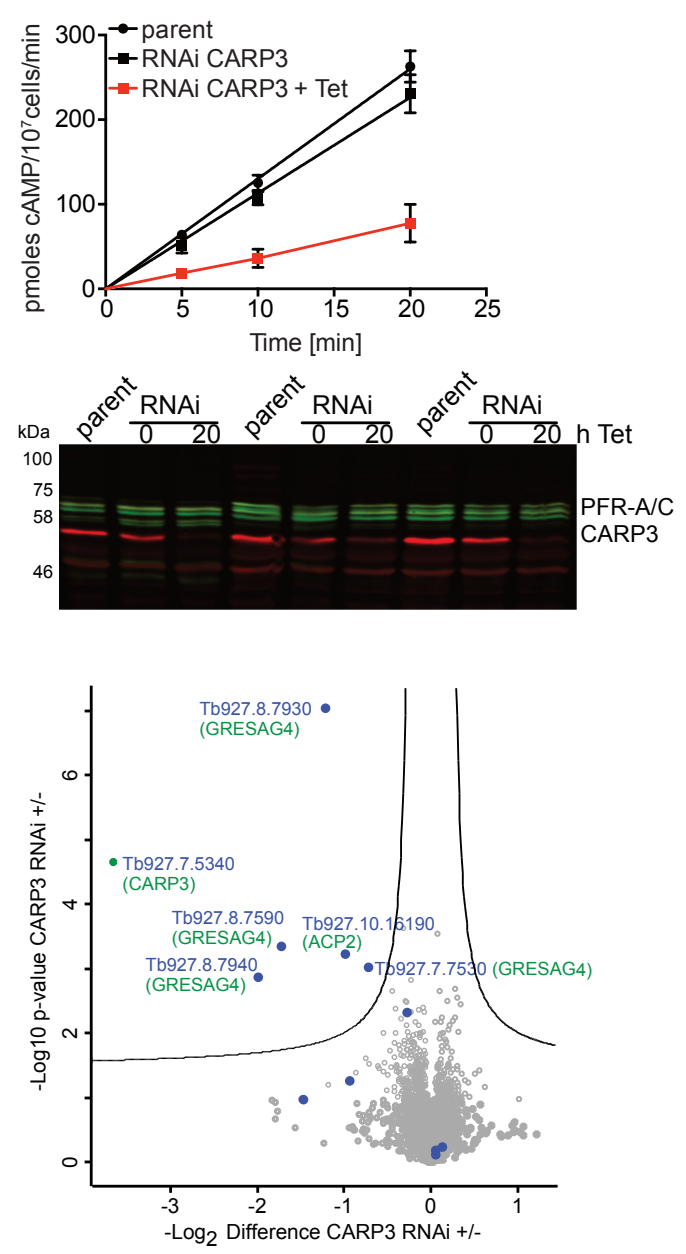

e

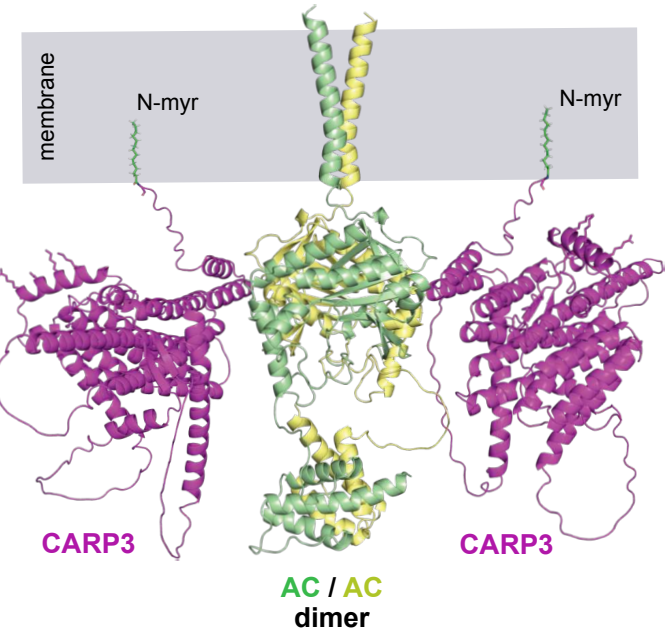

b

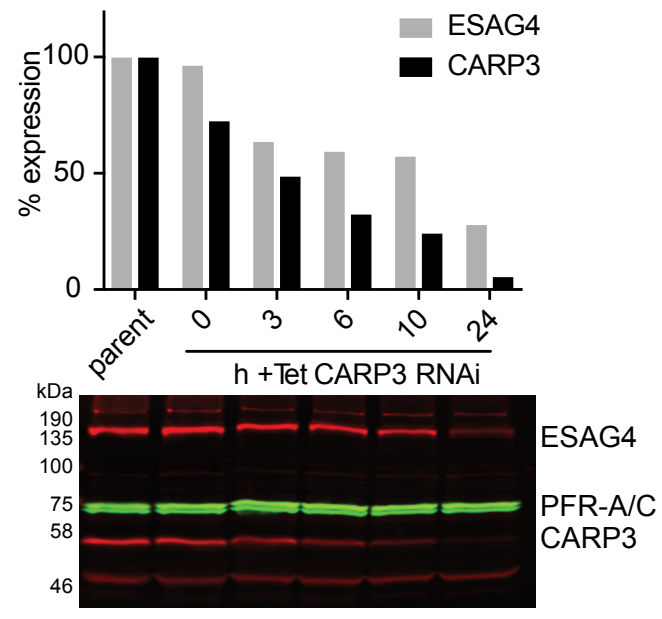

d
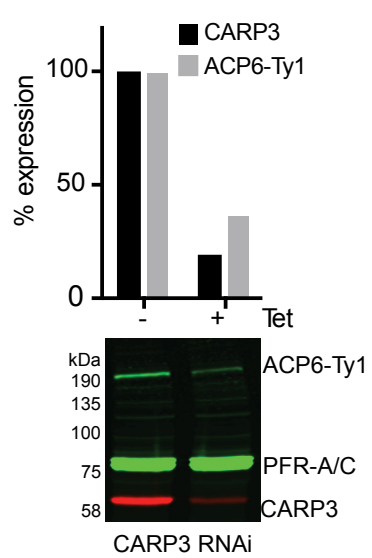

f

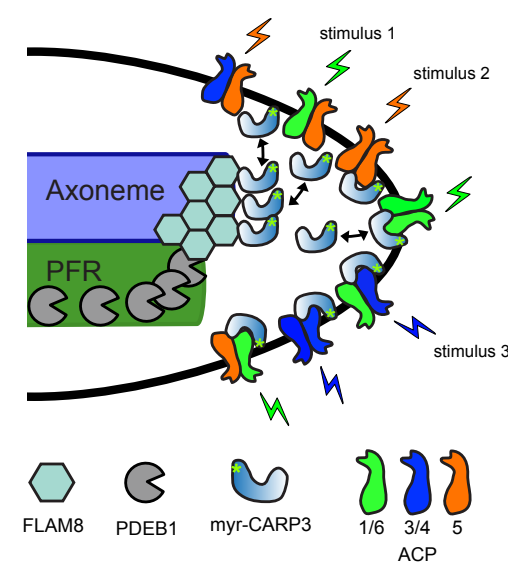

541 Fig. 6 CARP3 regulates AC abundance in BSFs and PCFs.

542 a, Total AC activity measured by swell dialysis assay in $T$. brucei MiTat 1.2 13-90 bloodstream forms 543 over $20 \mathrm{~min}$ upon Tet-inducible RNAi against CARP3 $( \pm 1 \mu \mathrm{g} / \mathrm{mL}$ Tet for $20 \mathrm{~h}$ ) with parental 13-90 as 544 control. Mean \pm SD of $n=9$ (3 independent replicates with 3 measurements each). CARP3 expression 545 levels were controlled by Western blot with PFR-A/C as loading control $(n=3$ replicates shown). 
b, Western blot analysis of CARP3 and ESAG4 in T. brucei MiTat 1.2 13-90 bloodstream forms upon Tet-inducible RNAi against CARP3 over $24 \mathrm{~h}$. CARP3 (anti-CARP3) and ESAG4 (anti-ESAG4) signals were normalized to the PFR-A/C loading control and protein expression in the parental cell line was set to $100 \%$.

550 c, Volcano plot representation of proteins identified by label-free proteomics in T. brucei MiTat 1.2 13-90 bloodstream forms upon Tet-inducible RNAi against CARP3 for $24 \mathrm{~h}(+)$ compared to non-induced (-).

552 Proteins are plotted according to $p$-value and fold change. Protein IDs that differ significantly ( $p$-value $\leq$ $\left.5530.05, s_{0}=0.1\right)$ in abundance are located above the significance line. AC isoforms are labeled as blue

554 dots. TriTrypDB entries are indicated for proteins with significantly altered abundance with CARP3 and

555 AC isoforms additionally labeled in green.

556 d, Western blot analysis of CARP3 and ACP6-Ty1 in procyclic forms of T. brucei 29-13 upon Tet-

557 inducible RNAi against CARP3 for 24 hours (+Tet) compared to non-induced (-Tet). ACP6 was fused to

558 a C-terminal Ty1 tag in this cell line. CARP3 (anti-CARP3) and ACP6-Ty1 (anti-Ty1) signals were

559 normalized to the PRF-A/C loading control and protein expression in the uninduced condition was set to $560100 \%$.

561 e, Cartoon representation of a model of the CARP3-AC complex at the membrane including the 562 transmembrane helix and the intracellular parts of an AC dimer (ESAG4). CARP3 N-myristoylation (N-

$563 \mathrm{myr}$ ) is indicated. The side chains of several basic amino acids of a membrane-proximal helix predicted 564 for CARP3 are shown. A second CARP3 molecule is shown here, as the AC dimer model sterically 565 allows interaction with two CARP3 molecules.

$566 \mathrm{f}$, Model illustrating the relative localization of CARP3, AC, FLAM8 and PDEB1 at the anterior tip of the 567 procyclic trypanosome flagellum. AC homo- and heterodimerization is indicated by different colors with 568 flagellar tip ACs grouped and colored according to their role in SoMo: ACP1/6 (green) inhibit SoMo, 569 ACP5 (orange) promotes SoMo and ACP3/4 (blue) were not linked to SoMo.

\section{Discussion}

573 In this work, we uncover a novel architecture of a cAMP signaling complex

574 essential for successful arthropod host-parasite interaction and hence

575 transmission of trypanosomes. CARP3, a protein with no sequence

576 homologue outside the genus Trypanosoma, interacts with and regulates

577 members of the receptor-type AC family. The model of the complex at the

578 flagellar tip membrane as illustrated in Fig. 6e, is based on orthogonal evidence from reciprocal pull down, BiolD proximity labeling, super-resolution 
microscopy and AlphaFold structure predictions. Phenotypes upon expression

581 of deletion mutants of CARP3 and the ESAG4 cyclase are in full agreement

582 with the high confidence prediction of an interface between the conserved AC

583 catalytic core domain and two N-terminal helices of CARP3. The overlay of

584 independent structure predictions of seven different AC isoforms in complex

585 with CARP3 shows remarkable similarity (Extended Data Fig. 8a,b), further

586 validating the structure prediction and suggesting that CARP3 is a pan-

587 cyclase interactor in trypanosomes. This is in agreement with identification of

588 multiple AC protein groups in CARP3-YFP pull-downs. The N-terminal

589 myristoylation of CARP3, revealed by the CARP3 $\triangle 3$ mutant and previous

590 chemical proteomics data ${ }^{45}$, is essential for tip localization and may enhance

591 AC complex formation by transient membrane interaction of CARP3. N-

592 myristoylation confers transient membrane association to proteins, but

593 membrane localization generally needs to be stabilized by a second

594 mechanism, either an interaction with a membrane protein, subsequent

595 palmitoylation or by ionic interactions of basic surface residues with the 596 membrane ${ }^{51,52}$. CARP3 has not been identified in a palmitoyl proteome ${ }^{53}$. The

597 structure model allows membrane contact of CARP3 by a helix rich in basic

598 residues and membrane insertion of a myristoyl group attached to CARP3

599 while in complex with ACs (Fig. 6e). Thus, a cooperative effect of AC-

600 interaction, $\mathrm{N}$-myristoylation and possibly basic surface residues is likely. A

601 possible mechanism to confine the complex to the tip membrane may be

602 association with lipid rafts that are highly enriched at the procyclic flagellar tip

603 membrane ${ }^{54}$. A lipid raft proteome of PCFs includes CARP3 ${ }^{55}$. In mammalian 
604 cells, association of ACs with lipid rafts has been described to be isoform-

605 specific. Whereas some mammalian AC isoforms are attracted to lipid rafts,

606 others are enriched outside raft microdomains ${ }^{56}$. A similar mechanism may

607 account for the observed differences in the subflagellar localization pattern of

608 different trypanosome AC isoforms ${ }^{18}$. It is conceivable that calpain 1.3 is also

609 part of the same membrane microdomain. Although we have no evidence for

610 a direct physical interaction with the AC-CARP3 complex, PALM microscopy

611 shows extensive colocalization of calpain 1.3 with CARP3 at single molecule

612 resolution at the procyclic flagellar tip, and BiolD with CARP3 as bait identifies

613 calpain 1.3 (83-fold enriched) in BSF. As calpains are linked to $\mathrm{Ca}^{2+}$ signaling

614 in other systems, calpain 1.3 may be involved in $\mathrm{Ca}^{2+}$-regulation of

615 trypanosome ACs, a property that has been reported earlier for some

616 trypanosome $\mathrm{ACs}^{57-60}$. Calpain 1.3 differs from classical calpains by lacking

617 some of the features critical for catalytic $\mathrm{Ca}^{2+-}$ dependent protease activity ${ }^{14}$.

618 CARP3 may serve a scaffolding role for recruitment of additional proteins in

619 AC signaling complexes. Significant hits in the BiolD interaction screen and

620 the presence of intrinsically disordered regions (IDR loops) on the cytoplasmic

621 face of the CARP3 structure model (Fig. 6e) are compatible with this

622 interpretation. IDRs often provide a platform for multiple weak interactions in

623 signaling complexes and are more likely to phase separate. An example is the

624 membrane associated pLAT-Grb2-Sos1 condensate in T-cell receptor

625 activation ${ }^{61}$. A fraction of CARP3 at the flagellar tip does not localize at the

626 membrane but colocalizes with the cytoskeletal scaffold protein FLAM8,

627 previously positioned at the plus end of the axonemal microtubules in PCF 15 
and also distributed along the flagellum in $\mathrm{BSF}^{62}$. The close proximity and

629 likely interaction of CARP3 and FLAM8 is supported by BiolD in BSF (125-fold

630 enrichment of FLAM8 with two biotinylation sites identified). Flagellar tip

631 localization of CARP3 is dependent on FLAM8; in its absence, CARP3 still

632 entered the flagellum but was not enriched at the tip. In the short epimastigote

633 stage, CARP3 also followed the FLAM8 redistribution along the length of the

634 flagellum ${ }^{62}$. FLAM8 therefore seems to provide a reservoir for CARP3 at the

635 flagellar tip in PCF, possibly to increase its local concentration. This may

636 indicate dynamic properties of the AC-CARP3 complex. The intraflagellar

637 transport (IFT) machinery is required for flagellar tip localization of FLAM863,

638 and possibly also of CARP3. The complex of CARP3 with AC isoforms was

639 detected in both stages (BSFs and PCFs). The proximity of CARP3 to FLAM8

640 and calpain 1.3, supported by super-resolution microscopy in PCF and by

641 BiolD in BSF, suggests that similar complexes are present in several stages

642 of the parasite's life cycle. So far, only phenotypes dependent on localization

643 at the procyclic flagellar tip have been observed. In contrast to the

644 aforementioned interactions, PDEB1 is not enriched in the tip microdomain

645 but forms a gradient with a higher concentration close to the flagellar tip and a

646 lower concentration at the flagellar base (Extended Data Fig. 2b). This

647 indicates a diffusion barrier function to establish a cAMP microdomain at the

648 tip in analogy to comprehensive studies in the mammalian system ${ }^{23}$.

649 CARP3 deletion completely blocks trypanosome SoMo, and

650 interestingly, the absence of CARP3 from the flagellar tip (in $\Delta$ flam8 and

651 carp3 $\Delta 3$ ) is alone sufficient for this phenotype. Genetic or pharmacological 
inhibition of PDEB1 was reported to abolish $\mathrm{SoMo}^{8}$ and a similar phenotype

653 seems to result from a single allele knock out (sKO) of the flagellar tip cyclase

654 ACP5 according to a recent preprint ${ }^{64}$. Therefore, genetic evidence indicates

655 that these proteins work together in a cAMP signaling pathway required for

656 SoMo. This pathway has been suggested to also control the parasite's ability

657 to orient and successfully migrate in the insect digestive tract to ultimately

658 reach the salivary glands and complete the infectious cycle ${ }^{9,65}$. In agreement

659 with this hypothesis, $\Delta$ carp3 and $\Delta$ flam8 mutants are unable to colonize the

660 salivary glands upon tsetse infections. We are aware of the complication that

661 colonization phenotypes can equally result from impact on the various

662 developmental transitions and adaptations along the parasite's journey in the

663 fly. Importantly, in contrast to previous studies, we provide a high degree of

664 phenotypic specificity: (1) Both $\Delta$ carp3 and $\Delta$ flam8 cell lines do not show any

665 growth or flagellar motility phenotypes in culture. (2) $\Delta$ carp3 can proceed

666 through complete differentiation in the RBP6-induced culture model with wild

667 type level of metacyclogenesis, thus excluding a cell autonomous defect of

668 the developmental program. Furthermore, the $\Delta$ flam8 cell line that delocalizes

669 CARP3 developed into epimastigote stages in vivo in the tsetse alimentary

670 tract. (3) Relocalization of CARP3 away from the tip by either deletion of

671 FLAM8 or by deletion of the myristoylation site in $C A R P 3$ is equally sufficient

672 to abolish SoMo. (4) For all phenotypic analyses, clones of the fly

673 transmissible pleomorphic AnTat 1.1 strain and freshly differentiated procyclic

674 forms of this strain were used. Fly transmissions were done in two different

675 laboratories with several independent $\mathrm{KO}$ lines and rescue lines. (5) Fly 
infection phenotypes are very similar for $\Delta$ carp3 and $\Delta$ flam8, although the

677 absolute infection rate differs between the AnTat 1.1 clones and fly colonies in

678 the two laboratories. Not a single trypanosome reached the salivary glands,

679 even in flies with positive cardia infection, yet both mutants were able to

680 colonize the cardia with reduced rate and at lower densities compared to WT.

681 In contrast, the $\Delta p d e b 1$ mutant $^{9}$ was not able to colonize the ectoperitropic

682 space and could not establish a cardia infection. The different phenotype may

683 indicate involvement of PDEB1 in additional steps of development or in

684 parasite fitness in the tsetse fly or be due to the inherent fly transmission

685 defect of the laboratory-adapted monomorphic Lister 427 strain $^{66-68}$ used by

686 Shaw, et al. ${ }^{9}$. Interestingly, in long trypomastigotes and long dividing

687 epimastigotes colonizing the anterior midgut, cardia and foregut, i.e. in the

688 migrating stages, FLAM8 is progressively enriched at the flagellar tip

689 microdomain (Fig. 3i; ${ }^{62}$ ), where CARP3-AC complexes are required. FLAM8

690 and consequently CARP3 are redistributed from the tip to the length of the

691 flagellum in short epimastigotes, a life cycle stage that normally attaches to

692 the salivary gland epithelium via the flagellar membrane ${ }^{69}$. The change in

693 subcellular localization may be cause or consequence of this attachment, but

694 the salivary gland colonization defect suggests a role of the tip signaling

695 complex prior to attachment. Together, $\Delta$ carp3 and $\Delta$ flam8 phenotypes

696 provide the best available evidence that an AC signaling complex controls a

697 crucial process leading to the colonization of fly salivary glands and thus

698 parasite transmission. In this context, the SoMo phenotype in vitro serves as

699 proxy for this complex process in vivo, rendering the signaling mechanisms 

experimentally more amenable. This is independent of the question whether

701 trypanosomes display true swarming, defined as directional motility of groups 702 of cells, mainly observed in prokaryotic systems 6 . A careful analysis of the 703 SoMo process on plates showed that single fluorescently labeled

704 trypanosomes in projections did not move directionally and single cell motility 705 did not correlate with movement of the projections ${ }^{70}$. In dissected tsetse fly 706 tissues, collective motion of trypanosomes as synchronized swarms with 707 coordinated flagellar beat were observed ex vivo ${ }^{11}$. Chemotaxis seems to be 708 an obvious link between the projections in vitro and the directional migration in 709 the tsetse alimentary tract. Chemotaxis has recently been described for 710 trypanosomes in vitro ${ }^{71}$. Metabolites derived from tsetse fly tissues or 711 endosymbionts of the tsetse and tissue surfaces have been suggested as

712 possible cues. A recent preprint demonstrated $\mathrm{pH}$ taxis of trypanosomes 713 undergoing SoMo on agarose plates ${ }^{64}$. Indeed, the trypanosomal receptor-

714 type ACs have been hypothesized to be activated by binding of ligands to 715 their extracellular part containing two VFT (Venus fly trap) domains ${ }^{26,72}$ 716 homologous to ligand-binding surface proteins in other systems ${ }^{73}$. However, 717 the membrane stress-induced activation of ACs by low osmolarity or low $718 \mathrm{pH}^{26,74-76}$ has not been linked to a specific domain or ligand binding event so 719 far. Membrane stress may also result from parasite attachment to insect host 720 tissue surfaces or cell-cell contacts in highly confined space, as observed for 721 swarming $P$. aeruginosa ${ }^{77}$. As some trypanosome ACs seem to be activated 722 by calcium in vivo ${ }^{57-60}$, the report of a membrane tension-mediated opening of 
723 a mechanosensitive ion channel in $T$. cruzi ${ }^{78}$ may merit follow-up in this 724 context.

725 Although the precise role of CARP3 in AC regulation remains to be

726 established, stabilization of several AC isoforms was obvious from rapid

727 decrease of AC abundance upon RNAi-mediated depletion of CARP3. Thus, 728 a role of the complex in AC receptor recycling or desensitization is a

729 reasonable hypothesis. Most AC interacting proteins reported so far in other 730 systems are regulators of $A C$ activity such as mammalian snapin ${ }^{79}$ or yeast 731 CAP1 ${ }^{80}$. However, $\mathrm{Hu}$, et al. ${ }^{81}$ reported a novel AC-interacting protein called 732 TRIP-Br1 (transcriptional regulator interacting with the PHD bromodomain 1) 733 that regulates $A C$ turnover by physically connecting multiple $A C$ isoforms to 734 the E3 ubiquitin ligase XIAP in mammalian cells. In turn, XIAP ubiquitinates a 735 conserved lysine residue in AC isoforms thereby accelerating endocytosis and 736 degradation. Previous studies have shown that the turnover of 737 transmembrane surface proteins in trypanosomes can be regulated by 738 ubiquitylation ${ }^{82,83}$, but the parasite's ACs were not among the proteins 739 downregulated upon individual depletion of two deubiquitylating enzymes.

740 Given the SoMo phenotypes observed in mutants of CARP3, of several tip 741 localized ACs, and of PDEB1, and the well-supported assumption that these 742 proteins all impact on SoMo via changes in cAMP concentration at the 743 flagellar tip [CAMPtip] or in the cAMP gradient along the flagellum, the 744 challenge is to integrate this information into a consistent model. It appears 745 contradictory that the $\triangle c a r p 3$ line and the sKO of $A C P 5^{64}$, both expected to 746 reduce [CAMPtip], abolish SoMo, while RNAi-mediated depletion of ACP1 or 
747 ACP6 (also expected to reduce [CAMPtip]) has apparently the opposite effect ${ }^{7}$.

748 The SoMo-negative phenotype of $\Delta p d e b 1$ (expected increase of [CAMP]) ${ }^{9}$ is

749 here interpreted as mechanistically distinct, as PDEB1 is not tip localized and

750 the mutant likely disrupts global [cAMP] and flagellar cAMP gradients. Based

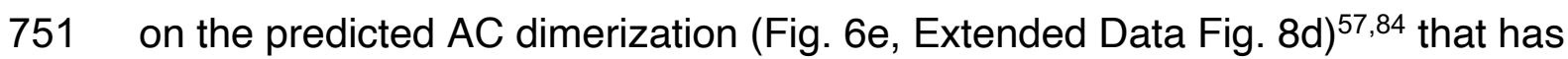

752 also been shown in vivo ${ }^{18,29,31}$, we propose a new model for AC regulation

753 (Fig. 6f). Dominant-negative heterodimer formation has previously been

754 described for invertebrate soluble guanylate cyclase $(\mathrm{GC})^{85}$, mammalian AC8

755 in vascular smooth muscle cells ${ }^{86}$ and also ESAG4 of trypanosomes ${ }^{31}$.

756 Different trypanosome AC isoforms may have different intrinsic cyclase

757 activities and may be activated or inhibited by different stimuli. Upon

758 heterodimerization, this may result in activation or dominant-negative

759 inhibition, dependent on $\mathrm{AC}$ isoform composition of the tip domain and

760 changing environmental stimuli to fine-tune cAMP levels. In agreement to the

761 model, AC isoform diversity and non-redundancy at the flagellar tip ${ }^{7}$ and

762 differential expression of transcripts encoding $A C$ isoforms in trypanosomes

763 colonizing midgut, cardia or salivary glands ${ }^{34,35}$ have been reported. CARP3

764 depletion might result in local compositional imbalance of AC isoforms

765 possibly due to isoform-specific quantitative differences in CARP3-mediated

766 complex stability. It is conceivable that $\mathrm{AC}$ complex signaling is required at

767 several locations and bottlenecks in the fly alimentary tract and that different

768 AC isoforms respond to different environmental cues. Our model implies that

769 future research should first focus on the AC family to unravel the CAMP

770 signaling initiated at the flagellar tip. Detailed biochemical and structural

771 analyses are required to identify AC-activating ligands and better understand 
772 the mechanism of cyclase activation, the role of homo- and heterodimerization

773 and the unique isoform diversity and stage regulation of the trypanosomal AC

774 family. Using the SoMo phenotype as simple in vitro assay, a key signaling

775 system coordinating host-parasite communication and securing successful

776 transmission of the parasite becomes accessible.

777

778

779 Methods

780 Trypanosome culture conditions

781 Bloodstream forms of the pleomorphic Trypanosoma brucei brucei strains

782 AnTat 1.1 'Munich' or AnTat 1.1E 'Paris' 39 were cultivated at $37^{\circ} \mathrm{C}$ and $5 \%$

$783 \mathrm{CO}_{2}$ in modified HMI-9 medium ${ }^{87}$ supplemented with $10 \%(\mathrm{v} / \mathrm{v})$ heat-

784 inactivated fetal bovine serum (FBS) and 1.1\% methylcellulose (only for AnTat

785 1.1'Munich'). Cell density was monitored using a haemocytometer and was

786 kept below $8 \times 10^{5} \mathrm{cells} / \mathrm{mL}$ for continuous growth of replicative long slender

787 bloodstream forms. Procyclic stage cells were generated by density-

788 dependent differentiation of long slender bloodstream forms to growth-

789 arrested short stumpy bloodstream forms (culture with starting density of $5 \times$

$79010^{5} \mathrm{cell} / \mathrm{mL}$ was grown for 36 hours without dilution). Short stumpy forms

791 were transferred into modified DTM medium ${ }^{88}$ complemented with $15 \%(\mathrm{v} / \mathrm{v})$

792 heat-inactivated FBS at $2 \times 10^{6} / \mathrm{mL}$, followed by addition of $6 \mathrm{mM}$ cis-

793 aconitate and cultivation at $27^{\circ} \mathrm{C}$. Procyclic forms of AnTat 1.1 'Munich', AnTat

794 1.1E 'Paris', AnTat 1.1 EATRO1125 T7T or MiTat 1.2 29-1389 were grown at 
$27^{\circ} \mathrm{C}$ in SDM-79 medium ${ }^{90}$ supplemented with $10 \%(\mathrm{v} / \mathrm{v})$ heat-inactivated FBS

796 and $20 \mathrm{mM}$ glycerol.

797

798 In vitro differentiation by RBP6 overexpression

799 Differentiation of procyclic forms by overexpression of RBP6 was performed 800 in EATRO 1125 T7T as described previously ${ }^{48,91 .}$

801

802 Cloning and generation of transgenic trypanosomes

803 Generation of a homozygous carp3 knock out:

804 Both CARP3 alleles were deleted from AnTat 1.1 bloodstream forms or

805 EATRO1125 procyclic forms carrying the RBP6 overexpression construct by

806 transfection with pTBT-based plasmids ${ }^{92}$ containing CARP3 5'UTR and

807 3'UTR sequences flanking a hygromycin or blasticidin resistance cassette

808 (kindly provided by Daniel Tagoe and Harry de Koning, Glasgow). Plasmids

809 were digested with Notl and Xhol for transfection according to standard

810 electroporation conditions ${ }^{93}$ and cells were selected with $2 \mu \mathrm{g} / \mathrm{mL}$ hygromycin

811 B or $2 \mu \mathrm{g} / \mathrm{mL}$ blasticidin, respectively.

812 Generation of endogenous CARP3 rescue and CARP3 3 rescue cell lines:

813 An endogenous CARP3 rescue cell line was generated by transfection of the

814 hemizygous carp3 KO cells resistant to hygromycin with a pEnT6B-based

815 plasmid $^{94}$ that contained the CARP3 5'UTR amplified with primers MG30 and

816 MG31 from genomic DNA as well as the first 300 nucleotides of the CARP3

817 ORF amplified with primers MG32 and MG33. The plasmid was linearized

818 with Notl for transfection and cells were selected with $2 \mu \mathrm{g} / \mathrm{mL}$ blasticidin. The 
same strategy was used for replacement of endogenous CARP3 by

820 CARP3 $\triangle 3$ using forward primer CARP3 $\Delta 3$ _HindIII_fw instead of MG30 for

821 amplification of the CARP3 ORF N-terminus with deletion of nucleotides 4-12.

822 Generation of a homozygous flam8 knock outs and rescue:

823 In AnTat 1.1E 'Paris' bloodstream forms, one flam8 allele was deleted and the

824 second one truncated to generate flam8 knock-outs sub-clones as described

$825 \mathrm{in}^{46}$. A rescue sequence was then re-introduced in the partially deleted allele

826 to produce an add-back strain ${ }^{46}$.

827 Tetracycline-inducible RNAi of CARP3:

828 Two copies of a tetracycline repressor were integrated into the T. brucei

829 AnTat 1.1 'Munich' genome by transfection with the Notl-linearized plasmid

$830 \mathrm{pHD1313^{95 }}$. Antibiotic selection was performed with $10 \mu \mathrm{g} / \mathrm{mL}$ phleomycin.

831 This cell line was further transfected with pHD615[PAC]CARP3_RNAi, a

832 plasmid allowing hairpin RNAi-mediated repression of CARP3. Two fragments

833 of the CARP3 ORF (nt 514 to 913; nt 514 to 965) were PCR-amplified from

834 AnTat 1.1 genomic DNA using primers CARP3_hairpin_up_HindIII and

835 CARP3_hairpin_sense_Xhol or CARP3_hairpin_up_BamHI and

836 CARP3_hairpin_antisense_Xhol, respectively, and cloned into pHD615[PAC]

837 by a three-component-ligation. Cells transfected with the Ncol-linearized

838 plasmid were selected with $0.1 \mu \mathrm{g} / \mathrm{mL}$ puromycin.

839 For generation of a tetracycline-inducible CARP3 RNAi cell line in procyclic

840 Lister 427 29-13, the plasmid p2t7-177[BLE]-CARP3 (Gould et al 2013) was

841 used for transfection and selection was carried out with $2.5 \mu \mathrm{g} / \mathrm{mL}$

842 phleomycin. 
844 The CARP3 C-terminus was amplified from genomic DNA (strain AnTat 1.1) 845 using primers p3074_CARP3_up_Swal and p3074_CARP3_low_BamHI and 846 cloned into the vector p307494 that enables C-terminal Ty1-tagging. The $4 x$

847 Ty1-tag was swapped to YFP from p3329 using BamHI and EcoRI. For PALM 848 microscopy, YFP from p3329 was replaced by mNeonGreen amplified from 849 plasmid pK19msB-mNeonGreen-ptsG ${ }^{96}$ by primers mNeon_BamHI_fw and 850 mNeon_EcoRI_rev. The plasmids were linearized with Notl and transfected 851 cells were grown in the presence of $2 \mu \mathrm{g} / \mathrm{mL}$ G418 or $0.1 \mu \mathrm{g} / \mathrm{mL}$ puromycin.

852 C-terminal in situ tagging of CARP3 with mCherry or photoactivatable

853 (PA)mCherry:

854 For C-terminal fusion of CARP3 to mCherry, the long primer PCR tagging 855 strategy was used ${ }^{97}$. YFP-Ty1 of plasmid pPOTv4 was replaced by mCherry856 Ty1 followed by PCR amplification of mCherry-TY with primers 857 pPOTv4_Lr_mCherry_TY_CARP3 fw and pPOTv4_Lr_mCherry_TY_CARP3 858 rev introducing stretches homologous to the $\mathrm{C}$-terminus of the CARP3 ORF 859 and the start of the CARP3 3'UTR. The PCR product was purified by phenol860 chloroform extraction prior to transfection and selection was done with 2 $861 \mu \mathrm{g} / \mathrm{mL}$ G418. For C-terminal fusion of CARP3 to photoactivatable 862 (PA)mCherry, the same strategy was used. mCherry-Ty1 was replaced by 863 PAmCherry amplified from plasmid pK19mobsacBparB-PAmCherry ${ }^{98}$ using 864 primers PAmCherry BamHI FWD and PAmCherry Sacl REV.

865 Overexpression of C-terminally Ty1-tagged CARP3 or CARP3 truncations. 
CARP3 C-terminus (171-end) were generated by PCR on genomic DNA of $T$.

868 brucei AnTat 1.1 using primers CARP3_fw_HindllI or CARP3_Ct_fw_HindllI

869 and CARP3_Ty1_Ct_rev_BamHI or CARP3_Ty1_Nt_rev_BamHI,

870 respectively, followed by ligation via BamHI/HindIII into the pTSARib

871 overexpression vector ${ }^{99}$ with a puromycin resistance cassette ${ }^{24}$. Constructs

872 were transfected into AnTat 1.1 CARP3 knockout cells and selected with 0.1

$873 \mu \mathrm{g} / \mathrm{mL}$ puromycin.

874 Inducible expression of CARP3-BirA*-Ty1 for proximity proteomics.

875 Primers CARP3_fw_HindIII and CARP3_NoSTOP_Spel were used to amplify

876 the CARP3 ORF that was inserted together with a downstream BirA*-Ty1

877 cassette via HindIII, Spel and BamHI restriction sites into plew100v5b1d-BLE,

878 a modified version of the original plew100 vector 89.

879 C-terminal in situ tagging of FLAM8 with YFP or mNeonGreen:

880 For C-terminal in situ tagging of FLAM8 with YFP, T. brucei AnTat 1.1 was

881 transfected with p3329.FLAM8 ${ }^{15}$ followed by selection with $0.1 \mu \mathrm{g} / \mathrm{mL}$

882 puromycin. For PALM microscopy, YFP was swapped to mNeonGreen using

$883 \mathrm{BamHI}$ and EcoRI. The plasmids were linearized with Nrul and transfected

884 cells were grown in the presence of $0.1 \mu \mathrm{g} / \mathrm{mL}$ puromycin.

885 C-terminal in situ tagging of calpain 1.3 with mNeonGreen:

886 For C-terminal in situ tagging of calpain 1.3 with mNeonGreen, the calpain 1.3

887 C-terminus was amplified from genomic DNA using primers

888 p3074_Calp1.3_fw and p3074_Calp1.3_rev and cloned into 
p3329.mNeonGreen via Kpnl and BamHI. The plasmid was linearized with

$890 \mathrm{Nrul}$ and transfected cells were selected with $0.1 \mu \mathrm{g} / \mathrm{mL}$ puromycin.

891 C-terminal in situ tagging of PDEB1 with mNeonGreen:

892 For C-terminal in situ tagging of PDEB1 with mNeonGreen, the PDEB1 C-

893 terminus was amplified from genomic DNA using primers PDEB1_Kpnl_fw

894 and PDEB1_BamHI_rev and cloned into p3329.mNeonGreen via Kpnl and

895 BamHI. The plasmid was partially digested with Ndel and transfected cells

896 were selected with $0.1 \mu \mathrm{g} / \mathrm{mL}$ puromycin.

897 C-terminal in situ tagging of $A C P 1, A C P 3, A C P 4, A C P 5$ or $A C P 6$ with Ty 1 or

898 mNeonGreen:

899 C-terminal Ty1 tagging of ACP1 or ACP6, respectively, was performed similar

900 to previously described by Saada et al. ${ }^{18}$ using the in situ tagging vector

$901 \mathrm{pMO}^{10} \mathrm{~T}^{100}$ and primers ACP1orfF/ACP1orfR and ACP1utrF/ACP1utrR or

902 ACP6orfF/ACP6orfR and ACP6utrF2/ACP6utrR2, respectively. The Ty1 tag

903 was swapped for mNeonGreen in plasmid pMO2T_ACP1 using primers

904 mNeon_Xhol_fw and mNeon_Sall_rev and Xhol/Sall digestion. ACP1 ORF

905 and 3'UTR fragments were replaced by Kpnl/Xhol or BamHI/Xbal swap,

906 respectively, with ACP3, ACP4 or ACP5 ORF and 3'UTR fragments using

907 primers ACP3_, ACP4_or ACP5_ORF_FW/REV and UTR_FW/REV,

908 respectively, resulting in plasmids pMO2mNG_ACP3, pMO2mNG_ACP4 and

909 pMO2mNG_ACP5. The plasmids were digested with EcoRV and Nsil

910 (pMO2mNG_ACP3), Kpnl and Pstl (pMO2mNG_ACP4) or Kpnl and Sphl for

911 transfection of procyclic AnTat 1.1 or $42729-13$ cells and selection was

912 carried out with $1 \mu \mathrm{g} / \mathrm{mL}$ puromycin. 
914 tag

915 The plasmid plew82.ESAG4 $\triangle$ CAT-2Ty1 (deletion of nucleotides 2680 to 3318

916 of ESAG4 ORF) was generated using the In-Fusion® HD Cloning Kit

917 (Takara), Phusion® High-Fidelity DNA Polymerase (NEB) and primers

918 DCAT_Mut1 and DCAT_Mut2 oriented in opposite directions with $19 \mathrm{bp}$

919 overlaps on the template vector plew82.ESAG4-2Ty1. The linear plasmid was

920 circularized in an In-Fusion reaction according to the manufacturer's

921 instructions. In order to obtain plew82.ESAG4 $\triangle$ CAT-GFP, a 733 bp

$922 \mathrm{Kpnl} / \mathrm{BamHI}$ fragment (containing EGFP) from plew82.ESAG3-DNi-2 ${ }^{31}$ was

923 inserted into the Kpnl/BamHI digested plew82.ESAG4 $\triangle$ CAT-2Ty1.

924 Linearization was done with Notl and transfected cells were selected with 2.5 $925 \mu \mathrm{g} / \mathrm{mL}$ phleomycin.

926 Constitutive overexpression of catalytically inactive ESAG4-GFP.

927 Catalytically inactive ESAG4 with point mutations D948A and R1052A fused

928 to a C-terminal GFP (ESAG4 DNi-3 from ${ }^{31}$ ) was cloned into the constitutive 929 expression vector pTSARib ${ }^{99}$ via HindIII and BamHI restriction sites. The 930 plasmid was linearized with Sphl and transfected into BSFs of $T$. brucei strain 931 MiTat 1.2 13-90 CARP3 RNAi ${ }^{30}$.

932 Constitutive overexpression of PKAR-GFP.

933 The N-terminus of $T$. brucei PKAR (protein kinase A regulatory subunit;

934 Tb927.11.4610, amino acids 1-200) was fused to C-terminal GFP and cloned 935 into the constitutive expression vector pTSARib $^{99}$ via a 3-component ligation 936 using HindIII and Eagl (PKAR fragment) and Eagl and BamHI (GFP) 
restriction sites. Linearization was done with Sphl and transfected cells were

938 selected with $2 \mu \mathrm{g} / \mathrm{mL}$ hygromycin B.

939 Plasmids for expression of CARP3 and ESAG4-2Ty1 in HEK 293 cells.

940 The ESAG4 C-terminus (nt 2424 to 3804) was amplified from a plasmid

941 containing the full ESAG4 (BES1/TAR40.13) ORF using primers

942 ESAG4_fwd_BstBI and ESAG4-2Ty1_rev_BamHI introducing a C-terminal

$9432 x T y 1$ tag. The fragment was ligated into plew82.ESAG4-DNi-1 ${ }^{31}$ cut with

944 BstBI and BamHI, resulting in plasmid plew82.ESAG4-2Ty1. ESAG4-2Ty1

945 was cut out with BamHI and HindIII and cloned into pcDNA3.1(+).

946 The CARP3 ORF was amplified from plasmid pTSARib.CARP3-Ty1 using

947 primers CARP3_fw_HindIII and CARP3_rev_Apal and cloned into

948 pcDNA3.1(+) via HindIII and Apal.

949 A list of all primers used in this study is provided as Supplementary Table 7.

950 All transfections were done in slender bloodstream forms, except for tagging 951 of ACPs.

952

953 Dual-luciferase ${ }^{\circledR}$ reporter assay in HEK cAMP reporter cells.

954 Human embryonic kidney cells 293T (HEK 293T) were cultured in DMEM/F12

955 medium (Lonza) supplemented with 10\% heat-inactivated FBS, $100 \mathrm{U} / \mathrm{mL}$

956 penicillin and $100 \mu \mathrm{g} / \mathrm{mL}$ streptomycin in a $5 \% \mathrm{CO}_{2}$ humidified incubator at

$95737^{\circ} \mathrm{C} .2 \times 10^{5} \mathrm{HEK} 293 \mathrm{~T}$ cells were plated into 12-well plates and transfected

958 after $24 \mathrm{~h}$ in triplicate with the plasmids pGL4.29[luc2P/CRE/Hygro]

959 (Promega), pRL-SV40 (Renilla Luciferase), pcDNA3.1(+)ESAG4-2Ty and

960 pcDNA3.1(+)CARP3 using Lipofectamine 2000 (Invitrogen) according to the 
manufacturer's instructions. The amount of plasmid DNA transfected per well

962 was optimized for each expression vector as follows: Renilla luciferase (5 ng),

963 Luc2P (250 ng), ESAG4 (250 ng) and CARP3 (62.5 to $500 \mathrm{ng}$ ). For the

964 different conditions, the amount of pcDNA3.1(+)CARP3 varied while keeping

965 a constant concentration of pcDNA3.1(+)ESAG4-2Ty1 (ESAG4:CARP3 ratios:

$9661: 0,1: 0.25,1: 0.5,1: 1,1: 2,0: 1)$. The total amount of DNA in each well was

967 adjusted to $1 \mu \mathrm{g}$ with the empty pcDNA3.1(+) vector. 24 hours after

968 transfection, cells were collected, and washed $2 \times$ with PBS. $5 \times 10^{4}$ cells were 969 harvested in passive lysis buffer (Promega) and the activities of the Firefly

970 and Renilla luciferases were measured sequentially using the Dual-

971 Luciferase ${ }^{\circledR}$ Reporter Assay System (Promega, cat. no. E1980).

972 Luminescence measurements were performed for $10 \mathrm{~s}$ at room temperature

973 by a Centro XS3 LB 960 luminometer (Bertold Technologies, Bad Wildbad,

974 Germany) provided with Mikrowin 2000 v.4.41 software (Mikrotek Labsis

975 Laborsysteme $\mathrm{GmbH}$ ). The light resulting from cleavage of the two substrates

976 was quantified as relative light units (RLU). Firefly:Renilla ratio was calculated

977 by dividing firefly RLU by the Renilla RLU. The assay was performed in

978 triplicate for each sample.

979 The rest of the cell pellets were resuspended in Laemmli buffer $\left(2.5 \times 10^{3}\right.$

980 cells $/ \mu \mathrm{L}$ ) and used for Western blot analysis.

981

982 AC assays

983 AC assays were performed according to Salmon et al. ${ }^{29}$ after mild acid (pH

984 5.5) treatment. Briefly, cultures of T. brucei MiTat 1.2 CARP3 RNAi cells were 
grown to a density of around $2 \times 10^{5}$ cells $/ \mathrm{mL}$, followed by RNAi induction with

$9861 \mu \mathrm{g} / \mathrm{mL}$ doxycycline for $24 \mathrm{~h}$. The cells were harvested at a density of $\sim 1 \times$

$98710^{6} \mathrm{cells} / \mathrm{mL}$ by centrifugation, washed twice with ice-cold phosphate-buffered 988 saline/glucose (PSG) buffer (137 mM NaCl, $2.7 \mathrm{mM} \mathrm{KCl}, 8.1 \mathrm{mM} \mathrm{Na}_{2} \mathrm{HPO}_{4}$, $9891.8 \mathrm{mM} \mathrm{KH}_{2} \mathrm{PO}_{4}, 1.5 \%$ glucose, $\mathrm{pH}$ 8.0) and after final counting resuspended 990 in "swell dialysis" buffer of low osmotic strength $(50 \mathrm{mM} \mathrm{KCl}, 5 \mathrm{mM} \mathrm{MgCl}, 1$ $991 \mathrm{mM}$ glucose, $1 \mathrm{mM}$ EGTA, 1X cOmplete EDTA-free protease inhibitor 992 cocktail, $13.3 \mathrm{mM}$ TES, pH 5.5) at a density of $5 \times 10^{8}$ cells $/ \mathrm{mL}$ for $1 \mathrm{~h}$ at $4{ }^{\circ} \mathrm{C}$. 993 The reaction was started by addition of $20 \mu \mathrm{L}$ sample $\left(1 \times 10^{7}\right.$ cells $)$ to $80 \mu \mathrm{L}$ 994 assay cocktail (0.5 mM cAMP, $10 \mathrm{mM}$ phosphocreatine, 50 units $/ \mathrm{mL}$ creatine 995 kinase, $1 \mathrm{mM} \mathrm{EGTA}, 10 \mathrm{mM} \mathrm{MgCl} 2,20 \mathrm{mM} \mathrm{KCl}, 0.5 \mathrm{mM}$ ATP, $1 \mathrm{mM}$ glucose, $9961 \times$ cOmplete EDTA-free protease inhibitor cocktail, $25 \mathrm{mM}$ TES pH 5.5 and $9970.8 \mu \mathrm{Ci}$ of [a-32P]-ATP at $10-40 \mathrm{Ci} / \mathrm{mmol}$ ) and was incubated for 5,10 and 20 $998 \mathrm{~min}$ at $37^{\circ} \mathrm{C}$. Each reaction was stopped by adding $100 \mu \mathrm{L}$ of stop solution 999 (2\% SDS, 40 mM ATP, $0.01 \mathrm{M}$ cAMP). [32P]cAMP was isolated by two-step 1000 chromatography according to Salomon et al. ${ }^{101}$ and measured by liquid 1001 scintillation counting. Each assay was carried out in triplicate. AC activity was 1002 calculated by linear regression analysis of the rate of cAMP production.

1003

1004 Cell motility assay

1005 Procyclic forms of $T$. brucei strain AnTat 1.1 were resuspended in $70 \%$ 1006 SDM79, $10 \% \mathrm{FCS}, 0.6 \%(\mathrm{v} / \mathrm{v})$ methylcellulose at a density of $8 \times 10^{6} \mathrm{cell} / \mathrm{s} / \mathrm{mL}$ 1007 and placed onto a glass slide. 12 regions of each slide were imaged on a 1008 Leica DMI600B series inverted microscope (Leica Microsystems). Cells were 
1009

1010

1011 included in the analysis. At least 50 cells were included for each cell line

1012 tested. Tracking and subsequent calculation of motion metrics was performed

1013 using the MTrackJ plugin for Image ${ }^{102}$. Statistical significance was

1014 determined by one-way ANOVA with $p \leq 0.05$ considered statistically

1015 significant.

1016 For the FLAM8 mutant panel, movies (150 frames, 50 ms exposure) were

1017 recorded in warm SDM79 medium with $1.1 \%$ methylcellulose at $1 \times 10^{6}$

1018 cells/mL under the $10 \times$ objective of an inverted DMI4000 LEICA microscope

1019 (Leica Microsystems) coupled to a Retiga-SRV camera (QImaging). Movies

1020 were converted with the MPEG Streamclip V.1.9b3 software (Squared 5) and

1021 analyzed with the MedeaLAB CASA Tracking V.5.5 software (Medea AV

$1022 \mathrm{GmbH})$, as described in Rotureau et al. ${ }^{103}$.

1023

1024 Social motility assay

1025 Agarose plates for social motility assays were prepared as described $5.5 \times$

$102610^{5}$ cells of $T$. brucei strain AnTat 1.1 or AnTat $1.1 \mathrm{E}$ were spotted in $5 \mu \mathrm{L}$ of

1027 SDM-79 on agarose plates within 7 days after density-dependent

1028 differentiation from bloodstream to procyclic stage.

\section{Generation of polyclonal antibodies}

1031 The CARP3 ORF was cloned as N-terminal His ${ }_{10}$ fusion into pETDuet-1 using

1032 primers Tb927.7.5340F10His and Tb927.7.5340_BamHI_rev via Ncol and 
1033 BamHI restriction sites and transformed into E. coli Rosetta. $500 \mu \mathrm{g}$ of His ${ }_{10-}$ 1034 CARP3 purified using a Ni-NTA column (Qiagen) were used for immunization 1035 of rabbits by Eurogentec, followed by further boosts with $500 \mu \mathrm{g}$ antigen. The 1036 CARP3 antiserum was affinity-purified using His ${ }_{10}-\mathrm{CARP} 3$ according to the 1037 method of Olmsted ${ }^{104}$.

1038

\section{In-gel fluorescence}

1040 In-gel fluorescence analysis was carried out as described previously ${ }^{49}$. Briefly, 1041 trypanosomes were lysed in $1 \times$ Laemmli sample buffer (125 mM Tris pH 6.8, $10424 \%(w / v)$ SDS, $20 \%(v / v)$ glycerol, 10\% 2-mercaptoethanol, 0.02\% (w/v) 1043 bromophenol blue) to a density of $3 \times 10^{6} / \mu \mathrm{L}$. Samples were sonicated 1044 (Bioruptor®, Diagenode (Belgium); settings: high energy, 4 cycles, $30 \mathrm{~s}$ 1045 on/off) and immediately subjected to 10\% SDS PAGE. The gel was scanned 1046 with a Typhoon Trio Variable Mode Imager System (GE Healthcare) at $\lambda_{\mathrm{ex}}=$ $1047488 \mathrm{~nm}$ and $\lambda_{\mathrm{em}}=526 \mathrm{~nm}$ for $\mathrm{mNeonGreen}$ and at $\lambda_{\mathrm{ex}}=670 \mathrm{~nm}$ and $\lambda_{\mathrm{em}}=$ $1048633 \mathrm{~nm}$ for visualization of the Blue Prestained Protein Standard (NEB). 1049

\section{Western blot}

1051 Western blot analysis was performed as previously described ${ }^{29}$. Primary 1052 antibodies used are anti-CARP3 (1:500), anti-ESAG4 ${ }^{29}$, anti-PFR-A/C ${ }^{105}$ 1053 (1:1000), anti-Ty1 ${ }^{106}$ (1:250), anti-PAD1 ${ }^{107}(1: 1000)$ and anti-RBP6 48 (1:1000). 1054 1055 Indirect immunofluorescence analysis 
1056 For microscopic analysis, trypanosomes were either spread on glass slides

1057 and fixed in methanol for $5 \mathrm{~min}$ at $-20^{\circ} \mathrm{C}$ or fixed in $2 \%$ PFA for $20 \mathrm{~min}$ at room 1058 temperature. Cellular DNA was visualized with 4',6-diamidino-2-phenylindole 1059 (DAPI; $1 \mu \mathrm{g} / \mathrm{mL}$ ). Image acquisition was performed with a DeltaVision Elite 1060 widefield fluorescence microscope (GE Healthcare) equipped with a 1061 CoolSnap HQ2 CCD camera (Photometrics, Arizona, USA) and images were 1062 processed with Image ${ }^{108,109}$. Primary antibodies used are anti-CARP3 1063 (1:150), anti-Ty1 (1:100), anti-SAXO110 (mAB25; 1:25), anti-EP procyclin 1064 (cedarlane, cat. no. CLP001AP, 1:500), anti-calflagin ${ }^{111}$ (1:1000). 1065 1066

\section{Live cell fluorescence microscopy}

1067 For live cell fluorescence microscopy, procyclic trypanosomes were 1068 resuspended in PBS and imaged using a DeltaVision Elite widefield 1069 fluorescence microscope (GE Healthcare) equipped with a CoolSnap HQ2 1070 CCD camera (Photometrics, Arizona, USA). Images were processed with 1071 Image ${ }^{108,109}$.

1072

\section{Photoactivated localization microscopy}

$10741.6 \times 10^{7}$ trypanosomes were fixed in $2 \%$ PFA for 20 min at room 1075 temperature, washed 2-4 times with PBS/10 mM glycine and resuspended in $107650 \mu \mathrm{L}$ PBS. $5 \mu \mathrm{L}$ fixed cells were mixed with $1.5 \mu \mathrm{L}$ (1:5000) $100 \mathrm{~nm}$ diameter 1077 fluorescent TetraSpeck Microbeads (Thermo Fisher Scientific) and $200 \mu \mathrm{L}$ 1078 PBS and loaded into an 8 well glass bottom $\mu$-slide (Ibidi, Martinsried, 1079 Germany). Slides were centrifuged for $10 \mathrm{~min}$ at $1400 \times \mathrm{g}$ and imaged on a 
1081 equipped with an Andor EM-CCD iXon DU 897 camera.

1082 PAmCherry was activated by an HR diode $50 \mathrm{~mW} 405 \mathrm{~nm}$ laser (Power 1083 linearly increased over the course of the experiment and dependent on the 1084 protein abundance) and excited via a $200 \mathrm{~mW} 561 \mathrm{~nm}$ OPSL laser line (15\% 1085 power) (Emission filter: LP 570). The 405 line trigger was set to "none" 1086 (continuous conversion) while the 561 line trigger was set to "integration" 1087 (laser active only when the camera is collecting photons). MNeonGreen was 1088 excited via a $200 \mathrm{~mW} 488 \mathrm{~nm}$ OPSL laser line (15\%) (no conversion required) 1089 (Emission filter: BP 495-590 + LP 750) (laser trigger set to "integration"). 1090 Fluorescent beads were bleached using a $561 \mathrm{~nm}$ laser at $100 \%$ power until 1091 the fluorescence level resided within the camera dynamic range. Both 1092 fluorescent tags were imaged in EPI mode (laser perpendicular to the sample) 1093 with an exposure time of $50 \mathrm{~ms}$ and an EMCCD gain of 200 (the experiment 1094 length depends of the protein abundance). 15 to 27 flagella were imaged per 1095 cell line. Peak fit, lateral drift and channel alignment were performed with ZEN 1096 Black (ZEN Black 2.1 SP3, version 14.0.4.201, 64-bit) while the follow up 1097 localization and colocalization analysis was performed via in house Fiji (Fiji is 1098 Just Image $\mathrm{J}^{108}$ ) and R scripts (http://www.R-project.org/) 1099 (https://github.com/GiacomoGiacomelli/Carp3-Co-localization-PALM). For 1100 colocalization analysis, we used the coordinate-based colocalization (CBC) 1101 method of Malkusch, et al. ${ }^{42}$. The CBC algorithm calculates a colocalization 1102 value for each molecule while taking into account the spatial distribution of the 1103 two populations within a user determined radius $\left(R_{\max }\right)$. All CBC values were 
1104 calculated for six different $R_{\max }(50,100.200,300,400,500 \mathrm{~nm})$ and radius

1105 intervals of $5 \mathrm{~nm}$ (Extended Data Fig. 3a-f). The resulting colocalization

1106 parameter varies between +1 (perfectly correlated / high probability of

1107 colocalization), through 0 (non-correlated / low probability of colocalization) to

$1108-1$ (anti-correlated / this value is of difficult interpretation) ${ }^{112,113}$. As CBC

1109 values are expression of the relative distribution of the two protein

1110 populations, the values are influenced by the detection efficiency of each

1111 fluorescent tag. We therefore generated a control cell line with one CARP3

1112 allele fused to PAmCherry and the other CARP3 allele fused to mNeonGreen.

1113 While both alleles were expressed at comparable levels, PALM imaging

1114 showed substantially more PAmCherry events compared to mNeonGreen

1115 events (Extended Data Fig. 2d). While mNeonGreen is suitable for PALM ${ }^{114}$, it

1116 is not photoactivatable and sample pre-bleaching is a necessary step for the

1117 collection of single molecule events. This results in permanent loss of a

1118 significant portion of the mNeonGreen population, hence altering the

1119 distribution of CBC values (Extended Data Fig. 2d). As CARP3 molecules are

1120 expected to colocalize with one another, we can use the obtained distribution

1121 of $\mathrm{CBC}$ values as a positive control for testing the remaining protein

1122 combinations. As a negative control, the CBC values' distribution for non-

1123 colocalizing proteins was obtained by simulating two independent Poisson

1124 point patterns (Extended Data Fig. 2f) via the "rpoispp" command ${ }^{115}$. The

1125 simulated point patterns were defined by a localization density $(\lambda$, expressed

1126 in localizations $/ \mathrm{nm}^{2}$ ) and an area ("owin" class object - see ${ }^{115}$ ). Specifically,

$1127 \lambda_{\text {PAmCherry }}\left(0.00013 \mathrm{loc} / \mathrm{nm}^{2}\right)$ and $\lambda_{\text {mNeonGreen }}\left(0.000026 \mathrm{loc} / \mathrm{nm}^{2}\right)$ were 
chosen as to possess the same localization density as the protein populations

1129 from the positive control, while the area confining the simulation are

1130 comprised of a rectangular structure of $1 \mu \mathrm{m}$ width and a total area equal to

1131 the total area from the positive control (broad approximation of a straight

1132 flagellum). The percentage of $\mathrm{CBC}$ values above or equal to 0.5

1133 (mNeonGreen to PAmCherry and vice versa) was determined and compared

1134 for increasing mNeonGreen concentrations $(25,50,100,200,400$ and 800

1135 molecules $/ \mu \mathrm{m}_{2}$ ) and constant PAmCherry concentration at an $R_{\max }$ of $300 \mathrm{~nm}$

1136 and an interval width of $10 \mathrm{~nm}$ (Extended Data Fig. 3g,h). This analysis

1137 revealed highly similar values for $\mathrm{CBC} \geq 0.5$ at the different $\mathrm{mNeonGreen}$

1138 concentrations, excluding the necessity of separate negative controls for each

1139 fluorescent protein pair. The percentage of $\mathrm{CBC}$ values above or equal to 0.5

1140 of CARP3-PAmCherry / ACP1-mNG, CARP3-PAmCherry / calpain1.3-mNG,

1141 CARP3-PAmCherry / FLAM8-mNG and CARP3-PAmCherry / CARP3-mNG

1142 was compared to the percentage of $\mathrm{CBC}$ values above or equal to 0.5 in the

1143 negative control $(p<0.05$, multiple comparison after Kruskal-Wallis, one

1144 tailed).

1145

1146 Pull-down with cAMP agarose

$11473 \times 10^{8}$ procyclic $T$. brucei cells were washed twice with PBS and lysed in 1148 lysis buffer (10 mM Tris/Cl pH 7.5; $150 \mathrm{mM} \mathrm{NaCl}$; $0.5 \mathrm{mM}$ EDTA; 0.5\% NP-40;

1149 Roche cOmplete protease inhibitor) for $30 \mathrm{~min}$ at $4^{\circ} \mathrm{C}$. Lysates were

1150 incubated with plain agarose beads (Biolog Bremen) for $1 \mathrm{~h}$ at $4^{\circ} \mathrm{C}$ in order to 1151 remove proteins binding non-specifically to the bead matrix. Pull-downs were 
performed by incubation of the pre-cleared lysates with $60 \mu \mathrm{L} 2$-AHA- or 8AHA-agarose (Biolog Bremen Cat. No. A054, A028) beads slurry for $2 \mathrm{~h}$ at $4^{\circ} \mathrm{C}$, followed by five washes with lysis buffer. Bound proteins were eluted by boiling (5 min $95^{\circ}$ ) with $40 \mu \mathrm{L} 2 \times$ Laemmli sample buffer.

\section{Immunoprecipitations}

Immunoprecipitation of CARP3 or of Ty1-tagged bait proteins was performed by binding anti-CARP3 or anti-Ty1 to magnetic protein A beads (Dynabeads, Invitrogen) followed by a 2-hour incubation with $1 \times 10^{8}$ trypanosomes lysed in lysis buffer (10 mM Tris/Cl pH 7.5; $150 \mathrm{mM} \mathrm{NaCl;} 0.5 \mathrm{mM}$ EDTA; 0.5\% NP40; Roche cOmplete protease inhibitor) for $30 \mathrm{~min}$ at $4^{\circ} \mathrm{C}$. Beads were washed $4 \mathrm{x}$ with lysis buffer and proteins were eluted by incubation with $50 \mu \mathrm{L}$ $2 \times$ Laemmli sample buffer for $5 \mathrm{~min}$ at $95^{\circ} \mathrm{C}$. Immunoprecipitations from HEK293T cells were carried out essentially using the same protocol with protein $A$ sepharose beads and $5 \times 10^{5}$ cells.

\section{Enrichment and mass spectrometry analysis of CARP3-interacting or}

\section{ESAG4-interacting proteins by GFP-trap pull-down}

Immunoprecipitation of CARP3-YFP or ESAG4-GFP was performed using a GFP nanobody covalently coupled to magnetic agarose beads (GFP-trap) according to the manufacturer's instructions (Chromotek, Martinsried, Germany). Briefly, $2 \times 10^{8}$ T. brucei cells (for CARP3-YFP pull-down: AnTat 1.1E wild type or CARP3-YFP, BSFs or PCFs, $n=2$ replicates each; for ESAG4-GFP pull-down: MiTat 1.2 13-90 or ESAG4-GFP, BSFs, $n=2$ 
replicates) were harvested by centrifugation, washed twice with serum-free

1177 culture medium and lysed in $1 \mathrm{~mL}$ lysis buffer (10 mM Tris/Cl pH 7.5; $150 \mathrm{mM}$

$1178 \mathrm{NaCl} ; 0.5$ mM EDTA; $0.4 \%$ NP-40 (ESAG4-GFP) or 0.5\% NP-40 (CARP3-

1179 YFP); Roche cOmplete protease inhibitor) for 30 min on ice. Soluble proteins

1180 were separated by centrifugation $\left(10 \mathrm{~min}, 20,000 \times \mathrm{g}, 4^{\circ} \mathrm{C}\right)$ and incubated

1181 with GFP-trap beads ( $25 \mu \mathrm{L}$ beads slurry) for $1 \mathrm{~h}$ at $4^{\circ} \mathrm{C}$ on an overhead

1182 rotator. Beads were washed $4 \mathrm{x}$ with lysis buffer, followed by three washes

1183 with $50 \mathrm{mM}$ Tris/Cl pH 8. Bound proteins were either eluted by boiling for 10

1184 min with Laemmli sample buffer for SDS PAGE or beads with bound proteins

1185 were subjected to on-bead digest for mass spectrometry analysis. After on-

1186 bead digestion with trypsin, digested peptides were separated on an Ultimate

11873000 RSLCnano (ThermoFisher) with a gradient from 4 to $40 \%$ acetonitrile in

$11880.1 \%$ formic acid over $40 \mathrm{~min}$ at $300 \mathrm{~nL} / \mathrm{min}$ in a $15-\mathrm{cm}$ analytical $(75 \mu \mathrm{m}$ ID

1189 home-packed with ReproSil-Pur C18-AQ 2.4 $\mu \mathrm{m}$ from Dr. Maisch). The

1190 effluent from the HPLC was directly electrosprayed into a Q Exactive HF

1191 instrument operated in data dependent mode to automatically switch between

1192 full scan MS and MS/MS acquisition. Survey full scan MS spectra (from $\mathrm{m} / \mathrm{z}$

1193 250-1600) were acquired with resolution $R=60000$ at $\mathrm{m} / \mathrm{z} 400$ (AGC target

1194 of $\left.3 \times 10^{6}\right)$. The ten most intense peptide ions with charge states between 2

1195 and 5 were sequentially isolated to a target value of $1 \times 10^{5}$ and fragmented at

$119627 \%$ normalized collision energy. Typical mass spectrometric conditions were:

1197 spray voltage, $1.5 \mathrm{kV}$; no sheath and auxiliary gas flow; heated capillary

1198 temperature, $250^{\circ} \mathrm{C}$; ion selection threshold, 33000 counts.

1199 Protein identification and quantification (iBAQ) was performed using 
MaxQuant 1.6.3.4 (CARP3-YFP) or 1.6.14.0 (ESAG4-GFP) ${ }^{116}$ with the

1201 following parameters: Database, TriTrypDB-

1202 42_TbruceiTREU927_AnnotatedProteins (CARP3-YFP pull-down) or 1203 TriTrypDB-48_TbruceiLISTER427_AnnotatedProteins (ESAG4-GFP pull1204 down); MS tol, 10 ppm; MS/MS tol, 20 ppm; Peptide FDR, 0.1; Protein FDR, 12050.01 Min. peptide Length, 5; Variable modifications, Oxidation (M); Fixed 1206 modifications, Carbamidomethyl (C); Peptides for protein quantitation, razor 1207 and unique; Min. peptides, 1; Min. ratio count, 2. Statistical analysis was 1208 performed using Perseus 1.6.7.0117 with the following workflow: proteins only 1209 identified by site, reverse hits or potential contaminants were filtered out. For 1210 ESAG4-GFP pull downs, three replicate LC-MS/MS runs of each sample were 1211 grouped as technical replicates and the median thereof was used for further 1212 calculations. The iBAQ values of the remaining proteins were $\log _{2}$ 1213 transformed and missing values were replaced from normal distribution. Only 1214 proteins identified in both pull down replicates were considered for statistical 1215 evaluation with an FDR $\leq 0.05$. The raw and processed mass spectrometry 1216 proteomics data have been deposited to the ProteomeXchange Consortium ${ }^{118}$ 1217 (http://proteomecentral.proteomexchange.org) via the PRIDE partner 1218 repository ${ }^{119}$ with the dataset identifiers PXD025398 (CARP3-YFP) and 1219 PXD025412 (ESAG4-GFP), respectively.

\section{Proximity proteomics using CARP3 BiolD}

1222 BiolD was adapted from ${ }^{120}$. Briefly, $4-5 \times 10^{8} \mathrm{~T}$. brucei MiTat $1.2 \mathrm{BSF}$ cells 1223 expressing tetracycline-induced $(1 \mu \mathrm{g} / \mathrm{mL}, 24 \mathrm{~h})$ CARP3-BirA ${ }^{*}-$ Ty 1 or CARP3 
1224 (control) were treated with $50 \mu \mathrm{M}$ biotin for $24 \mathrm{~h}$ ( $\mathrm{n}=3$ replicates). Cells were

1225 harvested by centrifugation, washed $3 \times$ with PBS and lysed in $500 \mu$ L lysis

1226 buffer (50 mM Tris/Cl pH 7.4; 500 mM NaCl; 5 mM EDTA; 0.4\% SDS; 1 mM

1227 DTT; Roche cOmplete protease inhibitor) by sonication (Bioruptor; 2 cycles

1228 with 30" on/off, high energy). Triton X-100 was added to a final concentration

1229 of $2 \%$, followed by sonication as above. Addition of $500 \mu \mathrm{L}$ Tris/Cl pH 7.4 was

1230 followed by another round of sonication. Soluble proteins were separated by

1231 centrifugation ( $15 \mathrm{~min}, 16,000 \times \mathrm{g}, 4^{\circ} \mathrm{C}$ ) and subjected to buffer exchange

1232 using a PD-10 column (GE Healthcare) according to the manufacturer's

1233 instructions. The eluate was concentrated (Spin-X UF 6 concentrator column)

1234 to $<1 \mathrm{~mL}$ and incubated with $50 \mu \mathrm{L}$ streptavidin-coupled magnetic beads

1235 (Dynabeads ${ }^{\circledR}$ MyOne ${ }^{\mathrm{TM}}$ Streptavidin T1, ThermoFisher) overnight at $4^{\circ} \mathrm{C}$ on

1236 an overhead rotator. Beads were washed 5x with PBS, followed by elution

1237 with Laemmli sample buffer for $10 \mathrm{~min}$ at $95^{\circ} \mathrm{C}$. Sample preparation for mass

1238 spectrometry, protein digestion and LC-MS parameters used for nanoLC-

1239 MS/MS analysis on a nanospray Q Exactive were previously described ${ }^{24}$.

1240 Raw spectra were analyzed with MaxQuant version 1.6.17.0116, which

1241 incorporates the Andromeda search engine, using the Trypanosoma brucei

1242 TriTrypDB-51_TbruceiTREU927 protein database and the following settings:

1243 three missed cleavages from trypsin digestion were allowed;

1244 carbamidomethyl-cysteine was set as fixed modification and biotinylation (K),

1245 oxidation (M), acetylation (protein $\mathrm{N}$-terminal) and deamidation $(\mathrm{N}, \mathrm{Q})$ were

1246 set as dynamic modifications. The MaxQuant output was loaded into Perseus

1247 version 1.6.7.0117 and filtered to exclude proteins 'only identified by site', 
reverse hits and potential contaminants. The LFQ values of the remaining

1249 proteins were $\log _{2}$ transformed and missing values were replaced from normal 1250 distribution. Only proteins identified in all three pull-downs were considered for 1251 further analysis. Proteins were considered as statistically significant with FDR $1252 \leq 0.05$ and $\mathrm{s}_{0}=2$ (two-sided Student's T-test). The raw and processed mass 1253 spectrometry proteomics data have been deposited to the ProteomeXchange 1254 Consortium ${ }^{118}$ (http://proteomecentral.proteomexchange.org) via the PRIDE 1255 partner repository ${ }^{119}$ with the dataset identifier PXD025357.

\section{Quantitative proteomics upon CARP3 knock-down}

$12583 \times 10^{7} \mathrm{~T}$. brucei MiTat 1.2 13-90 BSF cells with tetracycline-inducible CARP3 1259 RNAi knock-down were induced or not with $5 \mu \mathrm{g} / \mathrm{mL}$ tetracycline for $24 \mathrm{~h}(\mathrm{n}=$ 12604 replicates). Cell lysis, protein digestion, peptide purification and MS/MS 1261 analysis were performed as described by Humphrey et al. ${ }^{121}$. Purified 1262 peptides were injected in an RSLCnano system (Thermo) and separated in a 1263 25-cm analytical Aurora C18 nanocolumn (75 $\mu \mathrm{m}$ ID $120 \AA ̊$, $1.6 \mu \mathrm{m}$, Ion 1264 Opticks) with a 120-min gradient from 4 to $40 \%$ acetonitrile in $0.1 \%$ formic 1265 acid. The effluent from the HPLC was directly electrosprayed into a $Q$ 1266 Exactive HF (Thermo), operated in data dependent mode to automatically 1267 switch between full scan MS and MS/MS acquisition. Survey full scan MS 1268 spectra (from $\mathrm{m} / \mathrm{z} 375-1600$ ) were acquired with resolution $\mathrm{R}=60000 \mathrm{at} \mathrm{m} / \mathrm{z}$ 400 (AGC target of $3 \times 10^{6}$ ). The ten most intense peptide ions with charge

1270 states between 3 and 5 were sequentially isolated to a target value of $1 \times 10^{5}$ 1271 and fragmented at $27 \%$ normalized collision energy. Typical mass 
spectrometric conditions were: spray voltage, $1.5 \mathrm{kV}$; no sheath and auxiliary

1273 gas flow; heated capillary temperature, $250^{\circ} \mathrm{C}$; ion selection threshold, 33000

1274 counts.

1275 Protein identification and quantification (LFQ) was performed using MaxQuant 1276 version 1.6.10.43 ${ }^{116}$ with the following parameters: Database, TriTrypDB-

1277 46_TbruceiTREU927_AnnotatedProteins; MS tol, 10 ppm; MS/MS tol, 20 1278 ppm; Peptide FDR, 0.1; Protein FDR, 0.01 Min. peptide Length, 5; Variable 1279 modifications, Oxidation (M); Fixed modifications, Carbamidomethyl (C); 1280 Peptides for protein quantitation, razor and unique; Min. peptides, 1; Min. ratio 1281 count, 2. Statistical analysis was performed using Perseus 1.6.7.0 ${ }^{117}$ with the 1282 following workflow: proteins only identified by site, reverse hits or potential 1283 contaminants were filtered out. Only proteins that were identified in at least 1284 three of the four non-induced replicate samples were considered for further 1285 analysis. The LFQ values of the remaining proteins were $\log _{2}$ transformed and 1286 missing values were replaced from normal distribution. Statistical significance 1287 was evaluated with a two-sided Student's t-test with FDR $\leq 0.05$ and $\mathrm{s}_{0}=0.1$. 1288 The raw and processed mass spectrometry proteomics data have been 1289 deposited to the ProteomeXchange Consortium ${ }^{118}$ 1290 (http://proteomecentral.proteomexchange.org) via the PRIDE partner 1291 repository ${ }^{119}$ with the dataset identifier PXD025401.

1293 Tsetse fly infections and imaging of isolated parasites

1294 Fly infection experiments for flam8 KO and in situ FLAM8 rescue: 
1295 As previously described ${ }^{122}$, Glossina morsitans morsitans tsetse flies were

1296 maintained in the Trypanosome Transmission Group's insectarium of the

1297 Institut Pasteur at $27^{\circ} \mathrm{C}$ with $70 \%$ relative humidity. Flies were kept in

1298 Roubaud cages and fed through an artificial membrane feeding system with

1299 fresh mechanically defibrinated sheep blood three times a week. Batches of

130050 teneral males (unfed adults emerged from their puparium since $12 \mathrm{~h}$ to 72

1301 h) were allowed to ingest parasites in SDM79 medium supplemented with

$130210 \%$ fetal calf serum and $10 \mathrm{mM} \mathrm{L-glutathione.}$

1303 Flies were starved for at least $48 \mathrm{~h}$ before dissection. Four weeks after

1304 infection, all living flies were dissected: salivary glands were first rapidly

1305 isolated in a drop of PBS, the whole tsetse alimentary tract was then arranged

1306 lengthways and the foregut and proventriculus were physically separated from

1307 the midgut in distinct PBS drops. The infection was scrutinized in the posterior

1308 and anterior midgut (PMG and $\mathrm{AMG}$ ), in the cardia or proventriculus (PV) and

1309 foregut, as well as in the salivary glands (SG). The relative parasite densities

1310 per organ were evaluated by eye scoring under a $40 \times$ objective using the

1311 following scale: 0 for no parasites, 1 for 1 to 10 parasites, 2 for 10 to 100,3 for

1312100 to 1000 and 4 for $>1000$ parasites per microscopic field, as described in

1313 Schuster et al. ${ }^{11}$. Then, tissues were dilacerated to allow parasites to spread

1314 in PBS and parasites were recovered and treated for further experiments no

1315 more than 15 min after dissection. For immunofluorescence, parasites were

1316 rapidly allowed to settle on poly-lysine coated slides until drying. Cells were

1317 fixed for $10 \mathrm{sec}$ in methanol at $-20^{\circ} \mathrm{C}$ and re-hydrated in PBS during $10 \mathrm{~min}$.

1318 Slides were then incubated for $45 \mathrm{~min}$ at $37^{\circ} \mathrm{C}$ with the anti-FLAM8 $(1: 500)$ 
and anti-CARP3 (1:150) primary antibody in PBS with $0,1 \%$ bovine serum

1320 albumin. Slides were washed in PBS and incubated for 30 min at $37^{\circ} \mathrm{C}$ with

1321 the appropriate subclass-specific secondary antibodies coupled to Alexa Fluor 1322488 (Invitrogen) or Cy3 (Jackson ImmunoResearch). Slides were then stained 1323 with DAPI for visualization of their kinetoplast and nuclear DNA contents and 1324 mounted under coverslip with Prolong antifade reagent (Invitrogen). Slides 1325 were finally observed with a DMI4000 epifluorescence microscope (Leica) and 1326 images were captured with an Orca 03-G camera (Hamamatsu). Pictures 1327 were acquired with Micro-Manager 1.4 and prepared with ImageJ 1.8.0 (NIH).

1328 Antwerp fly infection experiments for carp3 $\mathrm{KO}$ and in situ CARP3 rescue:

1329 Freshly emerged tsetse flies (Glossina morsitans morsitans - Institute of 1330 Tropical Medicine Antwerp colony; 24-48 hours after emergence) were fed 1331 their first blood meal on fresh defibrinated horse blood containing bloodstream 1332 forms from either the T. bruceiAnTat 1.1 WT, the carp3 KO (two independent 1333 clones KO1 and KO2) or an in situ CARP3 rescue clone (resc1), 1334 supplemented with $10 \mathrm{mM}$ reduced L-glutathione, as described in ${ }^{123}$. For this, 1335 bloodstream parasites present in the blood of cyclophosphamide1336 immunosuppressed mice (Endoxan) (ITM Animal Ethics Committee clearance 1337 nr. VPU2014-1) at 6-7 days post-infection were mixed with defibrinated horse 1338 blood to obtain around $10^{6}$ parasites $/ \mathrm{ml}$ in the initial tsetse fly blood meal.

1339 Flies were further fed every 2-3 days on uninfected defibrinated horse blood. 1340 At a defined period after infection, all living flies were dissected after at least 134148 hours of starvation, and different tissues were examined for parasite 1342 presence/density by phase-contrast microscopy. To determine in a first 
experiment the overall tsetse infection rates for the different cell lines, midgut

1344 and salivary glands were examined five weeks after infection. Then, to

1345 estimate in more detail the infection rates and the parasite density in the 1346 posterior \& anterior midgut and proventriculus, flies were dissected three

1347 weeks after the initial infective bloodmeal (without the supplement of L-

1348 glutathione). Parasite abundance was scored using the aforementioned 1349 method of Schuster, et al. ${ }^{11}$.

\section{Structural modeling of AC and CARP3 proteins}

1352 Structural modeling of CARP3 (Extended Data Fig. 1) and ESAG4 (Extended

1353 Data Fig. 8) was initially carried out using the ColabFold notebook

1354 AlphaFold2_Advanced

1355 (https://colab.research.google.com/github/sokrypton/ColabFold/blob/main/bet

1356 a/AlphaFold2 advanced.ipynb) utilizing the MMseqs2 database for generating

1357 multiple sequence alignments (MSA) ${ }^{124}$. Structural models were later

1358 confirmed by executing the originally released AlphaFold2 code along with the

1359 full sequence database ${ }^{37}$ using an installation on a local high-performing

1360 cluster. Although CARP3 is trypanosome-specific, AlphaFold2 retrieved 34

1361 unique sequence homologs for generating the MSA, which is just above the

1362 alignment depth cut-off of 30 sequences below which the model accuracy was

1363 found to decrease significantly ${ }^{37}$. Models of CARP3-AC complexes shown in

1364 Extended Data Fig. 8 were generated using AlphaFold Colab

1365 (https://colab.research.google.com/github/deepmind/alphafold/blob/main/note

1366 books/AlphaFold.ipynb), a slightly simplified version of AlphaFold v. 2.1.0 
trained on oligomeric protein structures for improved prediction of protein

1368 complexes ${ }^{50}$. AlphaFold Colab was also used to produce a model of homo-

1369 dimeric ESAG4 bound to CARP3 as shown in Fig. 6e. All superpositioning

1370 and figures of protein structures were created in PyMOL (The PyMOL

1371 Molecular Graphics System, version 2.5 Schrödinger, LLC.).

1372

1373

1374

Data availability

1375 The proteomics datasets are available in the PRIDE partner repository with

1376 the dataset identifiers PXD025398 (CARP3-YFP pull down), PXD025412

1377 (ESAG4-GFP pull down), PXD025357 (CARP3 BioID) and PXD025401

1378 (CARP3 RNAi quantitative proteomics).

1379

1380

1381 Code availability

1382 R scripts for colocalization analysis of PALM data are available at

1383 (https://github.com/GiacomoGiacomelli/Carp3-Co-localization-PALM).

1384

1385

1386

1387

1388

1389

1390 


\section{References}

1392

13931 Zuzarte-Luís, V. \& Mota, M. M. Parasite Sensing of Host Nutrients and Environmental 1394 Cues. Cell Host \& Microbe 23, 749-758,

1395 doi:https://doi.org/10.1016/j.chom.2018.05.018 (2018).

13962 Landfear, S. M. \& Zilberstein, D. Sensing What's Out There - Kinetoplastid Parasites. 1397 Trends in parasitology 35, 274-277, doi:10.1016/j.pt.2018.12.004 (2019).

13983 Walsh, B. \& Hill, K. L. Right place, right time: Environmental sensing and signal 1399 transduction directs cellular differentiation and motility in Trypanosoma brucei.

1400 Molecular microbiology 115, 930-941, doi:https://doi.org/10.1111/mmi.14682 (2021).

14014 Brun, R., Blum, J., Chappuis, F. \& Burri, C. Human African trypanosomiasis. Lancet 1402 375, 148-159, doi:10.1016/s0140-6736(09)60829-1 (2010).

14035 Oberholzer, M., Lopez, M. A., McLelland, B. T. \& Hill, K. L. Social Motility in African $1404 \quad$ Trypanosomes. PLoS Pathogens 6, e1000739, doi:10.1371/journal.ppat.1000739 1405 (2010).

14066 Kearns, D. B. A field guide to bacterial swarming motility. Nature reviews.

1407 Microbiology 8, 634-644, doi:10.1038/nrmicro2405 (2010).

14087 Lopez, M. A., Saada, E. A. \& Hill, K. L. Insect stage-specific adenylate cyclases

1409 regulate social motility in African trypanosomes. Eukaryot Cell 14, 104-112, 1410 doi:10.1128/ec.00217-14 (2015).

14118 Oberholzer, M., Saada, E. A. \& Hill, K. L. Cyclic AMP Regulates Social Behavior in 1412 African Trypanosomes. mBio 6, doi:10.1128/mBio.01954-14 (2015).

14139 Shaw, S. et al. Flagellar cAMP signaling controls trypanosome progression through 1414 host tissues. Nat Commun 10, 803, doi:10.1038/s41467-019-08696-y (2019).

141510 Rose, C. et al. Trypanosoma brucei colonizes the tsetse gut via an immature 1416 peritrophic matrix in the proventriculus. Nat Microbiol, doi:10.1038/s41564-020-0707$1417 \quad z(2020)$. 
141811 Schuster, S. et al. Developmental adaptations of trypanosome motility to the tsetse fly 1419 host environments unravel a multifaceted in vivo microswimmer system. Elife 6, 1420 doi:10.7554/eLife.27656 (2017).

$1421 \quad 12$ Balbach, M., Beckert, V., Hansen, J. N. \& Wachten, D. Shedding light on the role of 1422 CAMP in mammalian sperm physiology. Mol Cell Endocrinol 468, 111-120, 1423 doi:10.1016/j.mce.2017.11.008 (2018).

142413 Langousis, G. \& Hill, K. L. Motility and more: the flagellum of Trypanosoma brucei. $1425 \quad$ Nature reviews. Microbiology 12, 505-518, doi:10.1038/nrmicro3274 (2014).

142614 Liu, W., Apagyi, K., McLeavy, L. \& Ersfeld, K. Expression and cellular localisation of 1427 calpain-like proteins in Trypanosoma brucei. Molecular and biochemical parasitology $1428169,20-26$, doi:10.1016/j.molbiopara.2009.09.004 (2010).

142915 Subota, l. et al. Proteomic analysis of intact flagella of procyclic Trypanosoma brucei 1430 cells identifies novel flagellar proteins with unique sub-localization and dynamics.

$1431 \quad$ Molecular \& cellular proteomics : MCP 13, 1769-1786, 1432 doi:10.1074/mcp.M113.033357 (2014).

143316 Varga, V., Moreira-Leite, F., Portman, N. \& Gull, K. Protein diversity in discrete 1434 structures at the distal tip of the trypanosome flagellum. Proceedings of the National 1435 Academy of Sciences of the United States of America 114, E6546-e6555, 1436 doi:10.1073/pnas.1703553114 (2017).

143717 Vélez-Ramírez, D. E. et al. APEX2 Proximity Proteomics Resolves Flagellum 1438 Subdomains and Identifies Flagellum Tip-Specific Proteins in Trypanosoma brucei. $1439 \quad m S p h e r e$ 6, doi:10.1128/mSphere.01090-20 (2021).

144018 Saada, E. A. et al. Insect Stage-Specific Receptor Adenylate Cyclases Are Localized 1441 to Distinct Subdomains of the Trypanosoma brucei Flagellar Membrane. Eukaryotic 1442 Cell 13, 1064-1076, doi:10.1128/EC.00019-14 (2014).

144319 Buxton, I. L. \& Brunton, L. L. Compartments of cyclic AMP and protein kinase in 1444 mammalian cardiomyocytes. The Journal of biological chemistry 258, 10233-10239 1445 (1983). 
144620 Zaccolo, M., Zerio, A. \& Lobo, M. J. Subcellular Organization of the cAMP Signaling

1447 Pathway. Pharmacol Rev 73, 278-309, doi:10.1124/pharmrev.120.000086 (2021).

144821 Schou, K. B., Pedersen, L. B. \& Christensen, S. T. Ins and outs of GPCR signaling in 1449 primary cilia. EMBO reports 16, 1099-1113,

1450 doi:https://doi.org/10.15252/embr.201540530 (2015).

145122 Tschaikner, P., Enzler, F., Torres-Quesada, O., Aanstad, P. \& Stefan, E. Hedgehog

1452 and Gpr161: Regulating cAMP Signaling in the Primary Cilium. Cells 9, 118,

1453 doi:10.3390/cells9010118 (2020).

145423 Schleicher, K. \& Zaccolo, M. Defining a cellular map of cAMP nanodomains. Mol

$1455 \quad$ Pharmacol, mol.119.118869, doi:10.1124/mol.119.118869 (2020).

145624 Bachmaier, S. et al. Nucleoside analogue activators of cyclic AMP-independent

1457 protein kinase A of Trypanosoma. Nature Communications 10, 1421,

1458 doi:10.1038/s41467-019-09338-z (2019).

145925 Makin, L. \& Gluenz, E. cAMP signalling in trypanosomatids: role in pathogenesis and

$1460 \quad$ as a drug target. Trends in Parasitology 31, 373-379, doi:10.1016/j.pt.2015.04.014

$1461 \quad$ (2015).

146226 Salmon, D. Adenylate Cyclases of Trypanosoma brucei, Environmental Sensors and 1463 Controllers of Host Innate Immune Response. Pathogens (Basel, Switzerland) 7, 48, 1464 doi:10.3390/pathogens7020048 (2018).

146527 de Koning, H. P. et al. Pharmacological validation of Trypanosoma brucei

1466 phosphodiesterases as novel drug targets. The Journal of infectious diseases 206, 1467 229-237, doi:10.1093/infdis/jir857 (2012).

146828 Oberholzer, M. et al. The Trypanosoma brucei cAMP phosphodiesterases TbrPDEB1 1469 and TbrPDEB2: flagellar enzymes that are essential for parasite virulence. FASEB $1470 \quad 21,720-731(2007)$.

147129 Salmon, D. et al. Cytokinesis of Trypanosoma brucei bloodstream forms depends on 1472 expression of adenylyl cyclases of the ESAG4 or ESAG4-like subfamily. Molecular 1473 microbiology 84, 225-242, doi:10.1111/j.1365-2958.2012.08013.x (2012). 
147430 Gould, M. K. et al. Cyclic AMP effectors in African trypanosomes revealed by

1475 genome-scale RNA interference library screening for resistance to the phosphodiesterase inhibitor CpdA. Antimicrobial agents and chemotherapy 57, 48824893, doi:10.1128/AAC.00508-13 (2013).

147831 Salmon, D. et al. Adenylate cyclases of Trypanosoma brucei inhibit the innate immune response of the host. Science (New York, N.Y.) 337, 463-466, doi:10.1126/science.1222753 (2012).

$148132 \quad$ Hertz-Fowler, C. et al. Telomeric Expression Sites Are Highly Conserved in Trypanosoma brucei. PLOS ONE 3, e3527, doi:10.1371/journal.pone.0003527 (2008).

148433 Rotureau, B. \& Van Den Abbeele, J. Through the dark continent: African trypanosome development in the tsetse fly. Frontiers in Cellular and Infection Microbiology 3, 53, doi:10.3389/fcimb.2013.00053 (2013).

$148734 \quad$ Savage, A. F. et al. Transcriptome Profiling of Trypanosoma brucei Development in the Tsetse Fly Vector Glossina morsitans. PLoS ONE 11, e0168877, doi:10.1371/journal.pone.0168877 (2016). during establishment of infection and progression of Trypanosoma brucei brucei through its insect host. bioRxiv, 2021.2005.2026.445766, doi:10.1101/2021.05.26.445766 (2021).

149436 Dyer, N. A., Rose, C., Ejeh, N. O. \& Acosta-Serrano, A. Flying tryps: survival and 1495 maturation of trypanosomes in tsetse flies. Trends Parasitol 29, 188-196, doi:10.1016/j.pt.2013.02.003 (2013).

$149737 \quad$ Jumper, J. et al. Highly accurate protein structure prediction with AlphaFold. Nature $1498 \quad 596,583-589$, doi:10.1038/s41586-021-03819-2 (2021).

149938 Lopez, M. A., Nguyen, H. T., Oberholzer, M. \& Hill, K. L. Social parasites. Current 1500 Opinion in Microbiology 14, 642-648, doi:https://doi.org/10.1016/j.mib.2011.09.012 1501 (2011). 
150239 Bachmaier, S., Thanner, T. \& Boshart, M. Culturing and Transfection of Pleomorphic

1503 Trypanosoma brucei. Methods in molecular biology (Clifton, N.J.) 2116, 23-38,

1504 doi:10.1007/978-1-0716-0294-2_2 (2020).

150540 Dean, S., Sunter, J. D. \& Wheeler, R. J. TrypTag.org: A Trypanosome Genome-wide 1506 Protein Localisation Resource. Trends in parasitology 33, 80-82,

1507 doi:10.1016/j.pt.2016.10.009 (2017).

$150841 \quad$ Aksoy, S., Weiss, B. \& Attardo, G. Paratransgenesis applied for control of tsetse 1509 transmitted sleeping sickness. Advances in experimental medicine and biology 627, 1510 35-48, doi:10.1007/978-0-387-78225-6_3 (2008).

$151142 \quad$ Malkusch, S. et al. Coordinate-based colocalization analysis of single-molecule 1512 localization microscopy data. Histochemistry and cell biology 137, 1-10, 1513 doi:10.1007/s00418-011-0880-5 (2012).

$151443 \quad$ Emmer, B. T., Maric, D. \& Engman, D. M. Molecular mechanisms of protein and lipid 1515 targeting to ciliary membranes. Journal of cell science 123, 529, 1516 doi:10.1242/jcs.062968 (2010).

$151744 \quad$ Kelly, F. D., Sanchez, M. A. \& Landfear, S. M. Touching the Surface: Diverse Roles 1518 for the Flagellar Membrane in Kinetoplastid Parasites. Microbiology and Molecular 1519 Biology Reviews 84, e00079-00019, doi:10.1128/mmbr.00079-19 (2020).

152045 Wright, M. H., Paape, D., Price, H. P., Smith, D. F. \& Tate, E. W. Global Profiling and 1521 Inhibition of Protein Lipidation in Vector and Host Stages of the Sleeping Sickness 1522 Parasite Trypanosoma brucei. ACS Infectious Diseases 2, 427-441, 1523 doi:10.1021/acsinfecdis.6b00034 (2016).

152446 Calvo-Alvarez, E., Crouzols, A. \& Rotureau, B. FLAgellum Member 8 modulates 1525 extravascular trypanosome distribution in the mammalian host. bioRxiv, 1526 2021.2001.2008.425862, doi:10.1101/2021.01.08.425862 (2021).

152747 Calvo-Alvarez, E., Cren-Travaille, C., Crouzols, A. \& Rotureau, B. A new chimeric 1528 triple reporter fusion protein as a tool for in vitro and in vivo multimodal imaging to 1529 monitor the development of African trypanosomes and Leishmania parasites. Infection, genetics and evolution : journal of molecular epidemiology and evolutionary 

(2018).

48 Kolev, N. G., Ramey-Butler, K., Cross, G. A. M., Ullu, E. \& Tschudi, C. RNA-Binding Protein. Science (New York, N.Y.) 338, 1352-1353, doi:10.1126/science.1229641 (2012).

153749 Chiasson, D. et al. A unified multi-kingdom Golden Gate cloning platform. Scientific reports 9, 10131, doi:10.1038/s41598-019-46171-2 (2019).

153950 Evans, R. et al. Protein complex prediction with AlphaFold-Multimer. bioRxiv, $1540 \quad$ 2021.2010.2004.463034, doi:10.1101/2021.10.04.463034 (2021).

154151 Goldston, A. M., Sharma, A. I., Paul, K. S. \& Engman, D. M. Acylation in trypanosomatids: an essential process and potential drug target. Trends in parasitology 30, 350-360, doi:10.1016/j.pt.2014.05.003 (2014). and disease. Journal of chemical biology 3, 19-35, doi:10.1007/s12154-009-0032-8 (2009).

$154753 \quad$ Emmer, B. T. et al. Global Analysis of Protein Palmitoylation in African

1548 Trypanosomes. Eukaryotic Cell 10, 455, doi:10.1128/EC.00248-10 (2011).

154954 Sharma, A. I. et al. Sterol targeting drugs reveal life cycle stage-specific differences in 1550 trypanosome lipid rafts. Scientific reports 7, 9105, doi:10.1038/s41598-017-08770-9 (2017).

155255 Sharma, A. I., Olson, C. L. \& Engman, D. M. The Lipid Raft Proteome of African 1553 Trypanosomes Contains Many Flagellar Proteins. Pathogens (Basel, Switzerland) 6, 1554 doi:10.3390/pathogens6030039 (2017).

155556 Cooper, Dermot M. F. \& Tabbasum, Valentina G. Adenylate cyclase-centred 1556 microdomains. Biochemical Journal 462, 199-213, doi:10.1042/BJ20140560 \%J 1557 Biochemical Journal (2014). 
155857 Bieger, B. \& Essen, L. O. Structural analysis of adenylate cyclases from

1559 Trypanosoma brucei in their monomeric state. Embo j 20, 433-445,

1560 doi:10.1093/emboj/20.3.433 (2001).

$156158 \quad$ Paindavoine, P. et al. A gene from the variant surface glycoprotein expression site

1562 encodes one of several transmembrane adenylate cyclases located on the flagellum

1563 of Trypanosoma brucei. Mol Cell Biol 12, 1218-1225, doi:10.1128/mcb.12.3.1218-

$1564 \quad 1225.1992(1992)$.

156559 Rolin, S. et al. Stage-specific adenylate cyclase activity in Trypanosoma brucei. Exp

1566 Parasitol 71, 350-352, doi:10.1016/0014-4894(90)90041-a (1990).

$156760 \quad$ Voorheis, H. P. \& Martin, B. R. Characteristics of the calcium-mediated mechanism 1568 activating adenylate cyclase in Trypanosoma brucei. Eur J Biochem 116, 471-477, 1569 doi:10.1111/j.1432-1033.1981.tb05360.x (1981).

157061 Su, Q., Mehta, S. \& Zhang, J. Liquid-liquid phase separation: Orchestrating cell 1571 signaling through time and space. Molecular Cell 81, 4137-4146, 1572 doi:10.1016/j.molcel.2021.09.010 (2021).

157362 Calvo-Alvarez, E. et al. Redistribution of FLAgellar Member 8 during the trypanosome 1574 life cycle: Consequences for cell fate prediction. Cell Microbio/ 23, e13347, 1575 doi:10.1111/cmi.13347 (2021).

157663 Fort, C., Bonnefoy, S., Kohl, L. \& Bastin, P. Intraflagellar transport is required for the 1577 maintenance of the trypanosome flagellum composition but not its length. Journal of 1578 cell science 129, 3026-3041, doi:10.1242/jcs.188227 (2016).

157964 Shaw, S. et al. Cyclic AMP signalling and glucose metabolism mediate $\mathrm{pH}$ taxis by 1580 African trypanosomes. bioRxiv, 2021.2001.2001.424252, 1581 doi:10.1101/2021.01.01.424252 (2021).

158265 Imhof, S. \& Roditi, I. The Social Life of African Trypanosomes. Trends Parasitol 31, 1583 490-498, doi:10.1016/j.pt.2015.06.012 (2015).

158466 Herder, S. et al. Trypanosoma brucei 29-13 strain is inducible in but not permissive 1585 for the tsetse fly vector. Exp Parasitol 117, 111-114, 1586 doi:10.1016/j.exppara.2007.05.011 (2007). 
$158767 \quad$ Peacock, L., Ferris, V., Bailey, M. \& Gibson, W. Fly transmission and mating of 1588 Trypanosoma brucei brucei strain 427. Molecular and biochemical parasitology 160, 1589 100-106, doi:10.1016/j.molbiopara.2008.04.009 (2008).

$159068 \quad$ Schuster, S. et al. Unexpected plasticity in the life cycle of Trypanosoma brucei. Elife $1591 \quad$ 10, doi:10.7554/eLife.66028 (2021)

159269 Vickerman, K., Tetley, L., Hendry, K. A. \& Turner, C. M. Biology of African 1593 trypanosomes in the tsetse fly. Biol Cell 64, 109-119, doi:10.1016/02484900(88)90070-6 (1988). Krüger, T., Maus, K., Kreß, V., Meyer-Natus, E. \& Engstler, M. Single-cell motile behaviour of Trypanosoma brucei in thin-layered fluid collectives. The European Physical Journal E 44, 37, doi:10.1140/epje/s10189-021-00052-7 (2021). DeMarco, S. F., Saada, E. A., Lopez, M. A. \& Hill, K. L. Identification of Positive Chemotaxis in the Protozoan Pathogen Trypanosoma brucei. mSphere 5, doi:10.1128/mSphere.00685-20 (2020).

160172 Schwede, A., Macleod, O. J., MacGregor, P. \& Carrington, M. How Does the VSG $1602 \quad$ Coat of Bloodstream Form African Trypanosomes Interact with External Proteins? PLoS Pathog 11, e1005259, doi:10.1371/journal.ppat.1005259 (2015).

160473 Heldin, C.-H., Lu, B., Evans, R. \& Gutkind, J. S. Signals and Receptors. Cold Spring 1605 Harb Perspect Bio/ 8, a005900-a005900, doi:10.1101/cshperspect.a005900 (2016).

160674 Nolan, D. P., Rolin, S., Rodriguez, J. R., Van Den Abbeele, J. \& Pays, E. Slender and 1607 stumpy bloodstream forms of Trypanosoma brucei display a differential response to 1608 extracellular acidic and proteolytic stress. Eur J Biochem 267, 18-27, 1609 doi:10.1046/j.1432-1327.2000.00935.x (2000).

161075 Rolin, S. et al. Simultaneous but independent activation of adenylate cyclase and 1611 glycosylphosphatidylinositol-phospholipase $\mathrm{C}$ under stress conditions in 1612 Trypanosoma brucei. The Journal of biological chemistry 271, 10844-10852, 1613 doi:10.1074/jbc.271.18.10844 (1996). 
$161476 \quad$ Lander, N., Chiurillo, M. A. \& Docampo, R. Signaling pathways involved in

1615 environmental sensing in Trypanosoma cruzi. Molecular microbiology 115, 819-828,

1616 doi:https://doi.org/10.1111/mmi.14621 (2021).

161777 Ellison, C. \& Brun, Y. V. Mechanosensing: a regulation sensation. Curr Biol 25, R113-

1618 R115, doi:10.1016/j.cub.2014.12.026 (2015).

161978 Dave, N. et al. A novel mechanosensitive channel controls osmoregulation,

1620 differentiation, and infectivity in Trypanosoma cruzi. eLife 10, e67449,

1621 doi:10.7554/eLife.67449 (2021).

162279 Wang, S. C., Lin, J. T. \& Chern, Y. Novel regulation of adenylyl cyclases by direct 1623 protein-protein interactions: insights from snapin and ric8a. Neuro-Signals 17, 169$1624 \quad$ 180, doi:10.1159/000200076 (2009).

162580 Bahn, Y. S. \& Sundstrom, P. CAP1, an adenylate cyclase-associated protein gene, 1626 regulates bud-hypha transitions, filamentous growth, and cyclic AMP levels and is 1627 required for virulence of Candida albicans. J Bacteriol 183, 3211-3223, 1628 doi:10.1128/JB.183.10.3211-3223.2001 (2001).

$162981 \quad \mathrm{Hu}, \mathrm{W}$. et al. The complex of TRIP-Br1 and XIAP ubiquitinates and degrades multiple 1630 adenylyl cyclase isoforms. eLife 6, e28021, doi:10.7554/eLife.28021 (2017).

163182 Chung, W. L., Leung, K. F., Carrington, M. \& Field, M. C. Ubiquitylation is required for 1632 degradation of transmembrane surface proteins in trypanosomes. Traffic 1633 (Copenhagen, Denmark) 9, 1681-1697, doi:10.1111/j.1600-0854.2008.00785.x (2008).

163583 Zoltner, M., Leung, K. F., Alsford, S., Horn, D. \& Field, M. C. Modulation of the 1636 Surface Proteome through Multiple Ubiquitylation Pathways in African

1637 Trypanosomes. PLOS Pathogens 11, e1005236, doi:10.1371/journal.ppat.1005236 1638 (2015).

$163984 \quad$ Naula, C., Schaub, R., Leech, V., Melville, S. \& Seebeck, T. Spontaneous 1640 dimerization and leucine-zipper induced activation of the recombinant catalytic 1641 domain of a new adenylyl cyclase of Trypanosoma brucei, GRESAG4.4B. Molecular 

(2001).

164485 Morton, D. B. \& Anderson, E. J. MsGC-beta3 forms active homodimers and inactive heterodimers with NO-sensitive soluble guanylyl cyclase subunits. J Exp Biol 206, 937-947, doi:10.1242/jeb.00160 (2003).

Vallin, B. et al. Novel short isoforms of adenylyl cyclase as negative regulators of cAMP production. Biochimica et Biophysica Acta (BBA) - Molecular Cell Research 1865, 1326-1340, doi:https://doi.org/10.1016/j.bbamcr.2018.06.012 (2018). trypanosomes is controlled by a density sensing mechanism which signals cell cycle arrest via the cAMP pathway. Journal of cell science 110 ( Pt 21), 2661-2671 (1997). Vassella, E. \& Boshart, M. High molecular mass agarose matrix supports growth of bloodstream forms of pleomorphic Trypanosoma brucei strains in axenic culture. Molecular and biochemical parasitology 82, 91-105 (1996). system for conditional gene knock-outs and dominant-negative genetics in Trypanosoma brucei. Molecular and biochemical parasitology 99, 89-101 (1999).

166291 Wargnies, M. et al. Gluconeogenesis is essential for trypanosome development in the tsetse fly vector. PLOS Pathogens 14, e1007502, doi:10.1371/journal.ppat.1007502

166592 Cross, M. et al. J-binding protein increases the level and retention of the unusual base $\mathrm{J}$ in trypanosome DNA. Molecular microbiology 46, 37-47 (2002).

166793 Schumann Burkard, G., Jutzi, P. \& Roditi, I. Genome-wide RNAi screens in 1668 bloodstream form trypanosomes identify drug transporters. Molecular and biochemical parasitology 175, 91-94, doi:10.1016/j.molbiopara.2010.09.002 (2011). 
$167094 \quad$ Kelly, S. et al. Functional genomics in Trypanosoma brucei: a collection of vectors for 1671 the expression of tagged proteins from endogenous and ectopic gene loci. Molecular and biochemical parasitology 154, 103-109, doi:10.1016/j.molbiopara.2007.03.012

1673 (2007).

167495 Alibu, V. P., Storm, L., Haile, S., Clayton, C. \& Horn, D. A doubly inducible system for 1675 RNA interference and rapid RNAi plasmid construction in Trypanosoma brucei. Molecular and biochemical parasitology 139, 75-82, doi:10.1016/j.molbiopara.2004.10.002 (2005). Martins, G., Giacomelli, G., Goldbeck, O., Seibold, G. \& Bramkamp, M. Substrate dependent cluster density dynamics of Corynebacterium glutamicum phosphotransferase system permeases. Molecular microbiology 111, doi:10.1111/mmi.14224 (2019).

168297 Dean, S. et al. A toolkit enabling efficient, scalable and reproducible gene tagging in 1683 trypanosomatids. Open biology 5, 140197-140197, doi:10.1098/rsob.140197 (2015). Böhm, K. et al. Chromosome organization by a conserved condensin-ParB system in the actinobacterium Corynebacterium glutamicum. bioRxiv, 649749, doi:10.1101/649749 (2019).

99 Xong, H. V. et al. A VSG expression site-associated gene confers resistance to human serum in Trypanosoma rhodesiense. Cell 95, 839-846 (1998). mediated C-terminal in situ tagging of Trypanosoma bruceigenes. Molecular and biochemical parasitology 145, 117-120, doi:10.1016/j.molbiopara.2005.09.002 (2006).

1693101 Salomon, Y. Adenylate cyclase assay. Adv Cyclic Nucleotide Res 10, 35-55 (1979).

1694102 Meijering, E., Dzyubachyk, O. \& Smal, I. Methods for cell and particle tracking. 1695 Methods in enzymology 504, 183-200, doi:10.1016/b978-0-12-391857-4.00009-4 1696 (2012). 
1697103 Rotureau, B. et al. Flagellar adhesion in Trypanosoma brucei relies on interactions 1698 between different skeletal structures in the flagellum and cell body. Journal of cell science 127, 204-215, doi:10.1242/jcs.136424 (2014).

1700104 Olmsted, J. B. Affinity purification of antibodies from diazotized paper blots of 1701 heterogeneous protein samples. The Journal of biological chemistry 256, 11955-

1702 11957 (1981).

1703

105 Kohl, L., Sherwin, T. \& Gull, K. Assembly of the paraflagellar rod and the flagellum 1704 attachment zone complex during the Trypanosoma brucei cell cycle. The Journal of

106 Bastin, P., Bagherzadeh, Z., Matthews, K. R. \& Gull, K. A novel epitope tag system to 1707 study protein targeting and organelle biogenesis in Trypanosoma brucei. Molecular and biochemical parasitology 77, 235-239 (1996). conveys the trypanosome differentiation signal. Nature 459, 213-217,

108 Schindelin, J. et al. Fiji: an open-source platform for biological-image analysis. Nature methods 9, 676-682, doi:10.1038/nmeth.2019 (2012).

1714109 Schneider, C. A., Rasband, W. S. \& Eliceiri, K. W. NIH Image to Image J: 25 years of 1715 image analysis. Nature methods 9, 671-675 (2012).

1716110 Dacheux, D. et al. A MAP6-related protein is present in protozoa and is involved in 1717 flagellum motility. PloS one 7, e31344-e31344, doi:10.1371/journal.pone.0031344 1718 (2012).

1719111 Giroud, C. et al. Murine Models for Trypanosoma brucei gambiense disease 1720 progression--from silent to chronic infections and early brain tropism. PLoS Negl Trop 1721 Dis 3, e509-e509, doi:10.1371/journal.pntd.0000509 (2009).

1722112 Adler, J. \& Parmryd, I. Quantifying colocalization by correlation: the Pearson 1723 correlation coefficient is superior to the Mander's overlap coefficient. Cytometry. Part $1724 \quad A$ : the journal of the International Society for Analytical Cytology 77, 733-742, 1725 doi:10.1002/cyto.a.20896 (2010). 
113 Zinchuk, V. \& Zinchuk, O. Quantitative colocalization analysis of confocal fluorescence microscopy images. Current protocols in cell biology Chapter 4, Unit 4.19, doi:10.1002/0471143030.cb0419s39 (2008).

114 Stockmar, I. et al. Optimization of sample preparation and green color imaging using the $\mathrm{mNeonGreen} \mathrm{fluorescent} \mathrm{protein} \mathrm{in} \mathrm{bacterial} \mathrm{cells} \mathrm{for} \mathrm{photoactivated} \mathrm{localization}$ microscopy. Scientific reports 8, 10137, doi:10.1038/s41598-018-28472-0 (2018). Baddeley, A., Rubak, E. \& Turner, R. Spatial Point Patterns: Methodology and Applications with R. (Chapman and Hall/CRC, 2015). p.p.b.-range mass accuracies and proteome-wide protein quantification. Nature biotechnology 26, 1367-1372, doi:10.1038/nbt.1511 (2008).

Tyanova, S., Temu, T. \& Sinitcyn, P. The Perseus computational platform for comprehensive analysis of (prote)omics data. 13, 731-740, doi:10.1038/nmeth.3901 (2016).

118 Vizcaíno, J. A. et al. ProteomeXchange provides globally coordinated proteomics data submission and dissemination. Nature biotechnology 32, 223-226, doi:10.1038/nbt.2839 (2014). Vizcaíno, J. A. et al. 2016 update of the PRIDE database and its related tools. Nucleic Acids Research 44, D447-D456, doi:10.1093/nar/gkv1145 \%J Nucleic Acids Research (2015).

1749121 Humphrey, S. J., Karayel, O., James, D. E. \& Mann, M. High-throughput and highsensitivity phosphoproteomics with the EasyPhos platform. Nature protocols 13,

1752122 Rotureau, B., Subota, I., Buisson, J. \& Bastin, P. A new asymmetric division 1753 contributes to the continuous production of infective trypanosomes in the tsetse fly. 

(2012).

1756123 Caljon, G. et al. The Dermis as a Delivery Site of Trypanosoma brucei for Tsetse

1757 Flies. PLoS pathogens 12, e1005744-e1005744, doi:10.1371/journal.ppat.1005744

$1758 \quad$ (2016).

1759124 Mirdita, M., Ovchinnikov, S. \& Steinegger, M. ColabFold - Making protein folding

1760 accessible to all. bioRxiv, 2021.2008.2015.456425, doi:10.1101/2021.08.15.456425 (2021).

1762125 Schenk, R., Bachmaier, S., Bringaud, F. \& Boshart, M. Efficient flavinylation of 1763 glycosomal fumarate reductase by its own ApbE domain in Trypanosoma brucei. The 1764 FEBS Journa/ n/a, doi:https://doi.org/10.1111/febs.15812 (2021).

1765

1766 


\section{Acknowledgements}

We thank Samuel Dean, Jessica Street and George Githure for plasmids, Nicolai Kolev, Frederic Bringaud, Keith Matthews and Melanie Bonhivers for antibodies, and Frank Schwede (Biolog, Bremen) for cAMP beads. We thank Benoit Vanhollebeke and David Pérez Morga for facilities for AC assay and HEK cell experiments (ULB, IBBM), Harry de Koning and Daniel Tagoe for exchange of information and Aline Crouzols and Robin Schenk for research assistance.

The work was supported by a BioNa young scientists award of LMU to S.B., MC-IEF Fellowship PIEF-GA-2013-626034 to M.K.G., IN.WBI excellence fellowship to L.R.V., CNPq/Universal Grant 725 422022/2016-0 to D.S. and LMU core funding to M.Bo. Work on super-resolution imaging was supported by grants from the Deutsche Forschungsgemeinschaft (DFG, grant numbers 268759902 and 443931024$)$ to M.Br. B.R. was supported by the Institut Pasteur, the Institut National pour la Santé et le Recherche Médicale (INSERM), the French Government Investissement d'Avenir programme Laboratoire d'Excellence "Integrative Biology of Emerging Infectious Diseases" (ANR-10-LABX-62-IBEID) and the French National Agency for Scientific Research (projects ANR-14-CE14-0019-01 EnTrypa and ANR-18CE15-0012 TrypaDerm).

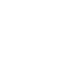


1791 Author Contributions

1792 S.B., B.R., D.S., J.V.D.A., M.Br., and M.Bo. designed and supervised

1793 research; S.B., E.C.-A., G.G., L.R.V., E.L., A.A., M.K.G., J.V.D.A., A.B.

1794 performed research; J-W.D., I.F., A.I. performed, analysed or supervised

1795 mass spectrometry; S.B., G.G., E.C.-A., L.R.V., A.A., E.L., B.R., M.Br., D.S.,

1796 J.V.D.A., M.Bo. analyzed data; S.B. and M.Bo. wrote the paper.

1797

1798

1799 Conflict of interest statement

1800 The authors declare no competing interests.

1801

1802

1803 Additional Information

1804

1805 Extended Data

1806 

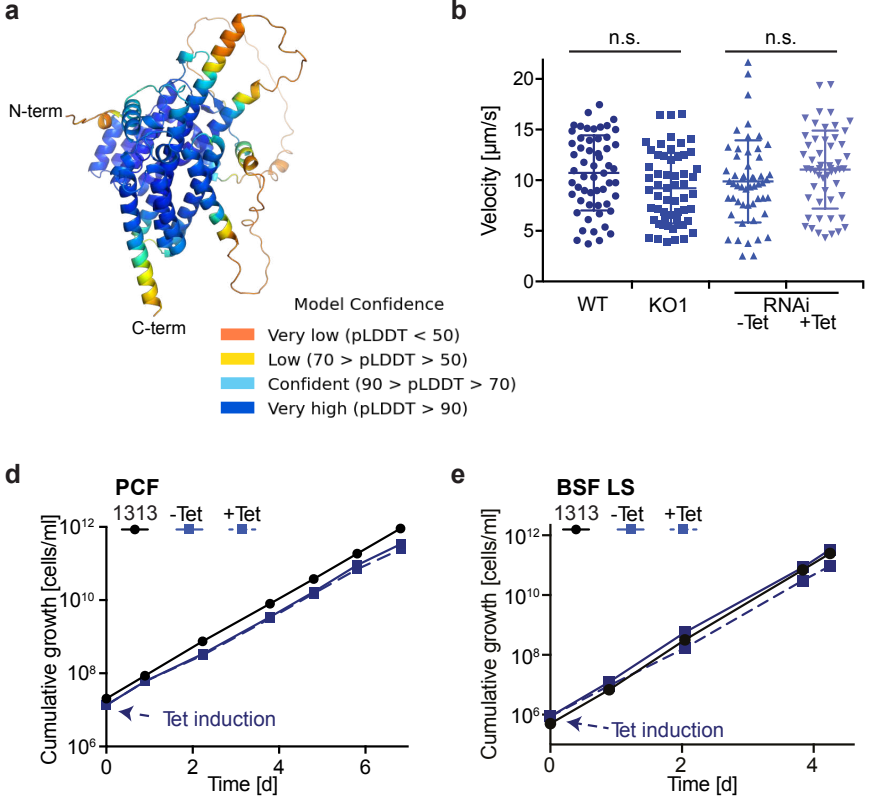

g

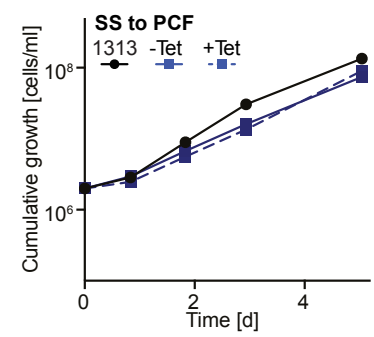

e

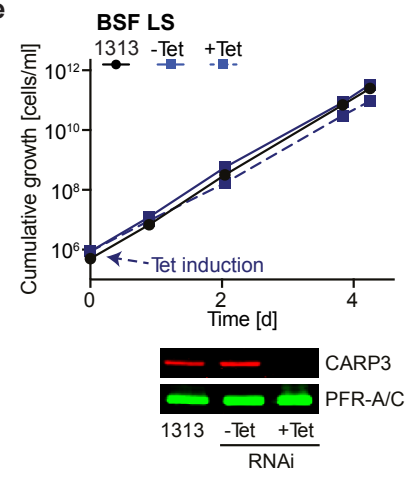

h

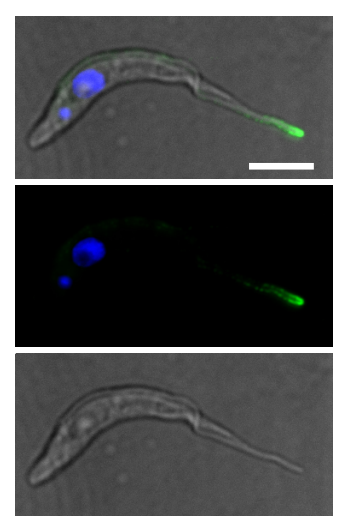

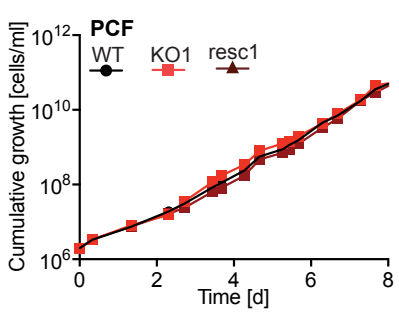

$\mathbf{f}$
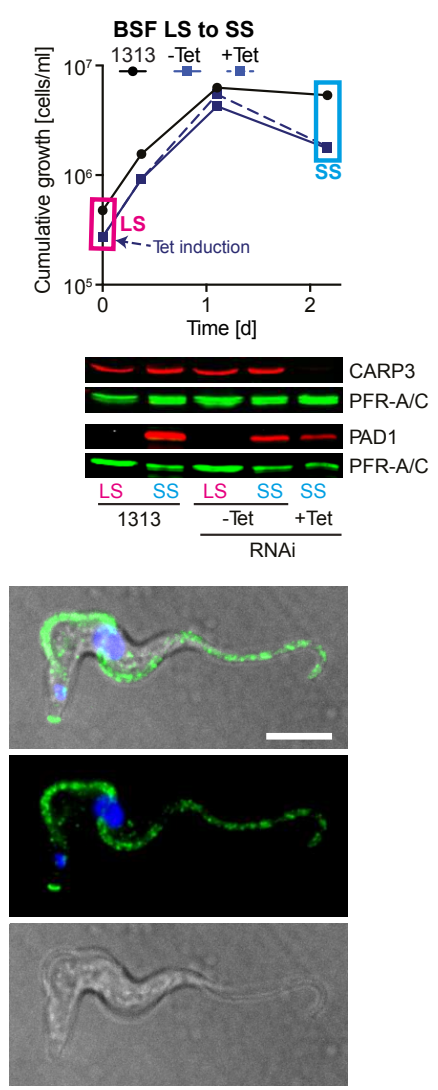

Extended Data Fig. 1 CARP3 is not essential for growth or differentiation of $T$. brucei but shows life

a, Cartoon representation of a predicted model of T. brucei CARP3 using AlphaFold ${ }^{37}$. Model confidence is a, Cartoon representation of a predicted model of T. brucei CARP3 using AlphaFold ${ }^{37}$. Model confidence is
illustrated using the predicted local-distance difference test (pLDDT) score, indicated by the color-coding. b. Analysis of single cell mean velocity (mean \pm SD; $n=56$ (WT), 57 (KO1), 53 (RNAi -Tet), 54 (RNAi +Tet)) of procyclic form (PCF) AnTat 1.1 WT, carp3 KO and RNAi cell lines. n.s.: non-significant ( $p>0.05$; ANOVA). c, Representative growth curves of PCF AnTat 1.1 WT, carp3 KO (KO1) and CARP3 rescue (resc1) cell lines. Corresponding Western blot is shown in Fig. $1 \mathrm{a}$.

d, e, Representative growth curves of PCF (d) or bloodstream form (BSF) (e) AnTat 1.11313 and tetracyclineinducible CARP3 RNAi cell lines. RNAi was induced by addition of $5 \mu \mathrm{g} / \mathrm{mL}$ tetracycline (+Tet condition). Repression of CARP3 levels was confirmed by Western blot analysis (e) with PFR-A/C as loading control. Western blot corresponding to $(d)$ is shown in Fig. $1 b$.

$\mathbf{f}, \mathbf{g}$, Growth of cell lines as in (d, e) during differentiation from BSF long slender (LS) to short stumpy (SS) (f) or during SS to PCF differentiation (g). Western blot in (f) shows expression of CARP3 and the stumpy marker protein PAD1. PFR-A/C serves as loading control. The growth curve in (f) was started at time point $0 \mathrm{~h}$ with long slender cells at a density of $5-8 \times 10^{3}$ cells $/ \mathrm{mL}$ and Tet induction of CARP3 RNAi. Growth was monitored over $52 \mathrm{~h}$ without culture dilution, resulting in development into PAD1-expressing short stumpy forms. The growth curve in $(\mathrm{g})$ was initiated with the SS cells from $(\mathrm{f})$.

$\mathbf{h}, \mathbf{i}$, Indirect immunofluorescence analysis of CARP3 (green) in T. brucei AnTat 1.1 procyclic forms (h) or long slender bloodstream forms (i). DNA was stained with DAPI (blue). Fluorescence channels were merged with the differential interference contrast (DIC). Scale bars $5 \mu \mathrm{m}$. 
a

CARP3-PAmCh

CARP3-PAmCh

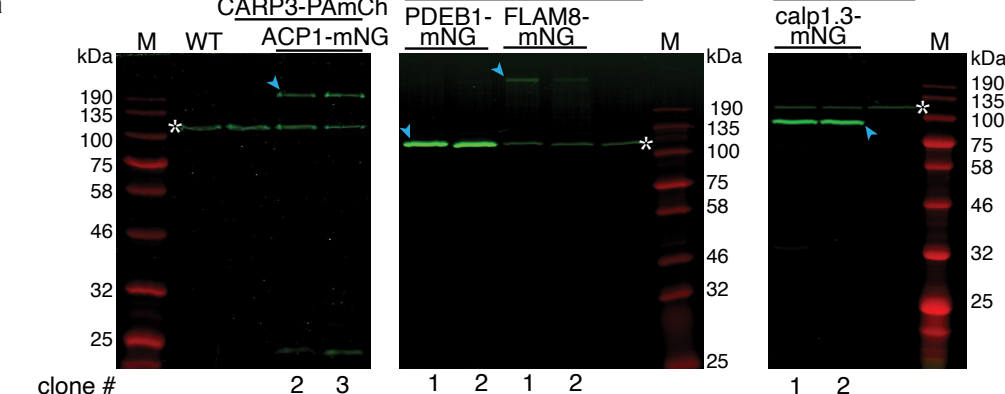

b
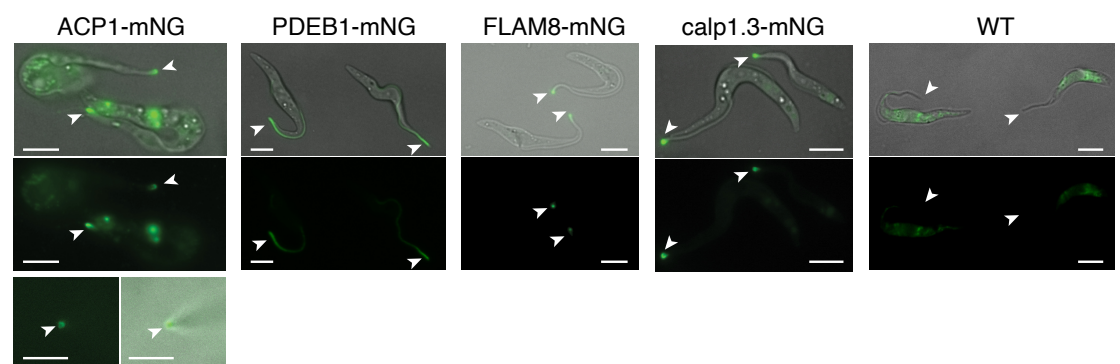

C
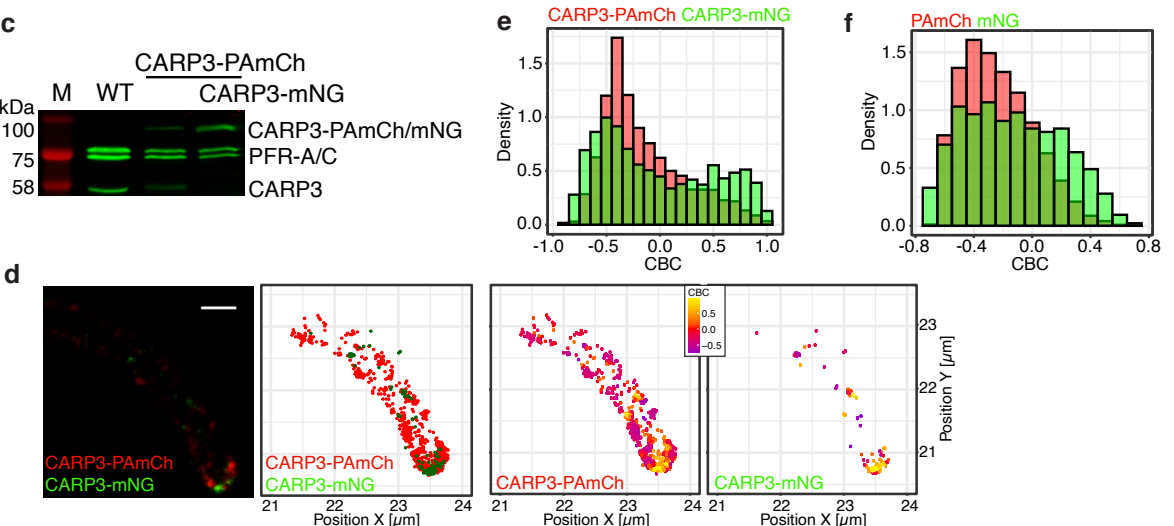

Extended Data Fig. 2 Generation of trypanosome cell lines for photoactivated localization microscopy of CARP3 and putative colocalization partners.

$\mathbf{a}$, In-gel fluorescence and $\mathbf{b}$, live cell fluorescence microscopy of procyclic T. brucei AnTat 1.1E expressing CARP3-PA-mCherry (PAmCh) and ACP1P, PDEB1, FLAM8, or calpain 1.3 (calp1.3), respectively, C-terminally

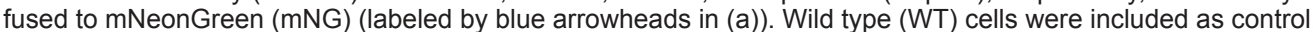
The white asterisk $\left({ }^{*}\right)$ marks an endogenous, autofluorescent protein that we identified as fumarate reductase ${ }^{124}$. Note that the PDEB1-mNG fusion protein runs at the same apparent molecular weight as the autofluorescent fumarate reductase. White arrowheads in (b) point towards flagellar tips. The second image shown for ACP1-mNG displays a fluorescent flagellar tip from the top perspective. Scale bars $5 \mu \mathrm{m}$. M protein molecular weight marker. c, Western blot analysis (anti-CARP3, anti-PFR-A/C (loading control)), d, PALM imaging and e, colocalization analysis (as in Fig. 2) of procyclic T. brucei AnTat 1.1E expressing CARP3-PAmCherry (PAmCh, red) and CARP3mNeonGreen (mNG, green). While WT cells express CARP3 ( 57 kDa) from two endogenous alleles (c, lane 'WT'), the hemizygous in situ CARP3-PAmCherry cells (middle lane in (c)) express CARP3 from one wild type allele $(\sim 57 \mathrm{kDa})$ and one endogenous CARP3-PAmCherry fusion ( $85 \mathrm{kDa})$. The CARP3-PAmCherry/CARP3$\mathrm{mNG}$ (c, right lane) cell line expresses two endogenously tagged CARP3 alleles, both resulting in proteins with similar molecular weight $(\sim 85 \mathrm{kDa})$, one fused to PAmCherry, the other fused to $\mathrm{mNG}$, resulting in replacement of both endogenous alleles. Scale bar in (d) $0.5 \mu \mathrm{m}$.

f, CBC values distributions for two simulated independent Poisson point patterns characterized by densities equal to the ones from procyclic T. brucei AnTat 1.1E expressing CARP3-PAmCherry and CARP3-mNeonGreen. The point patterns are confined within a rectangular area of $1 \mu \mathrm{m}$ width (approximation of a straight flagellum). 

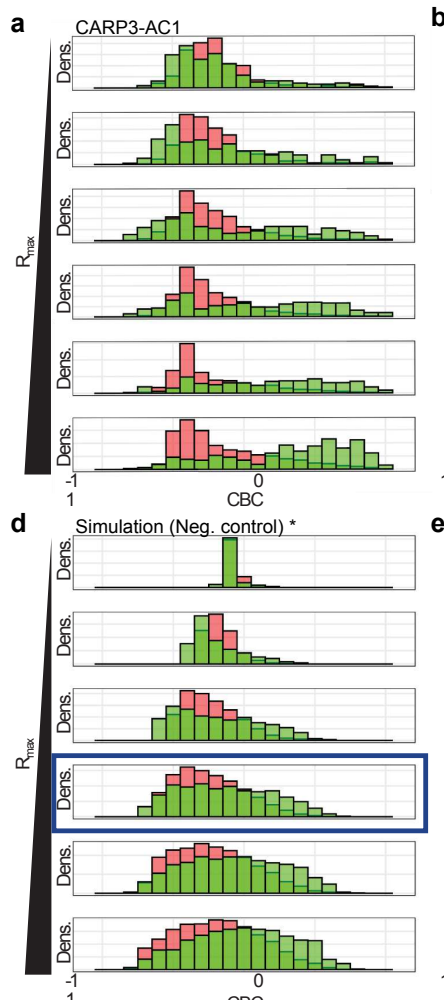

$\mathrm{CBC}$

g Simulation (Neq control-Interactor density) ** h

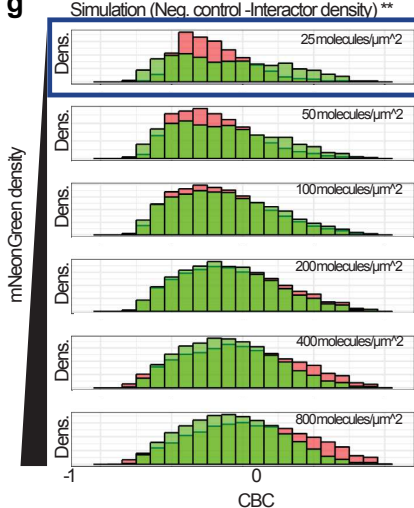

b CARP3-calp1.3

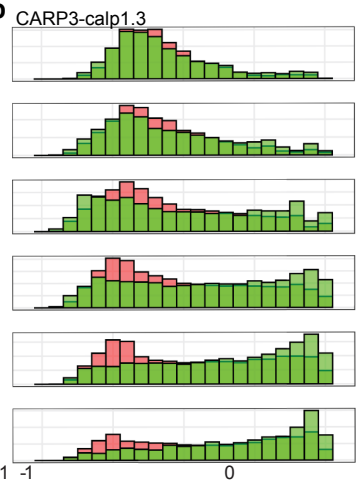

$\mathrm{CBC}$

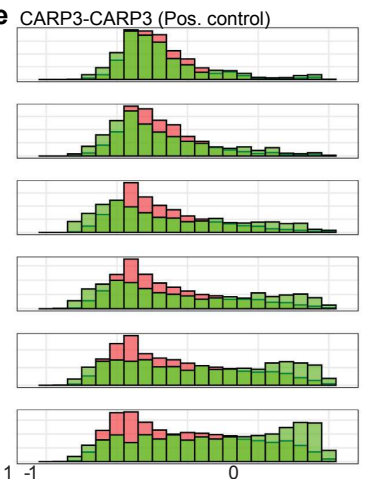

$\mathrm{CBC}$

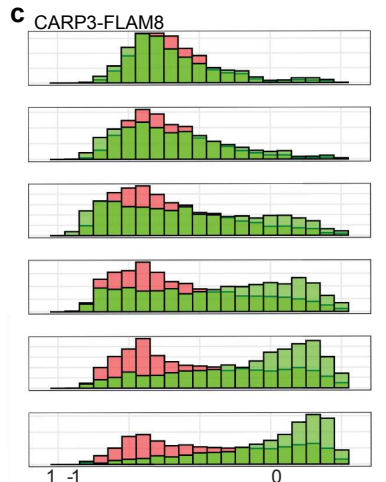

CBC

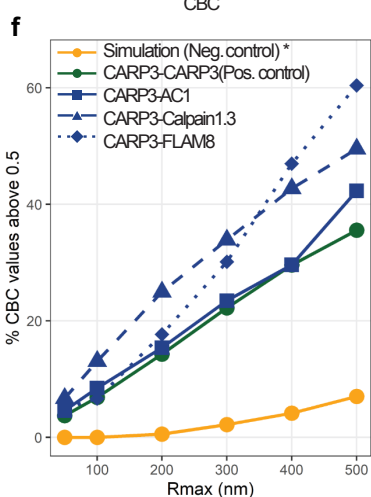

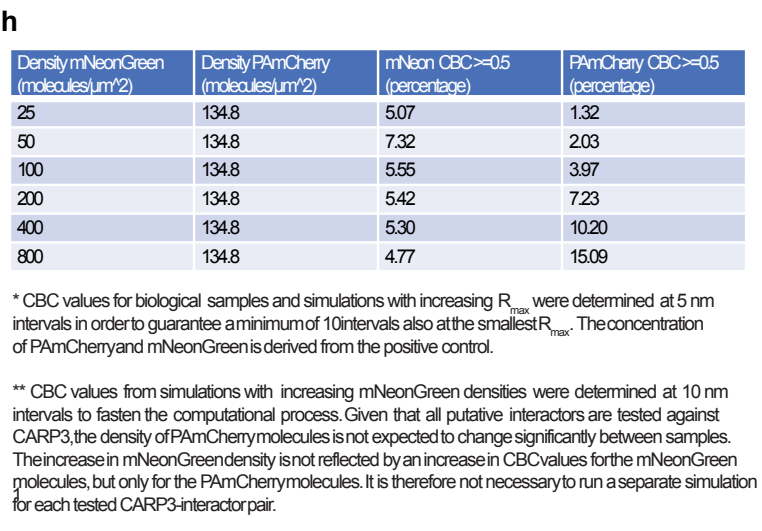

Extended Data Fig. 3 Coordinate-based colocalization (CBC) analysis of PALM data for CARP3 and putative colocalization partners.

a-e, CBC value density distributions for CARP3-AC1 (a), CARP3-calpain 1.3 (b), CARP3-FLAM8 (c), negative control (d) and CARP3-CARP3 (e) calculated for six different $R_{\max }(50,100,200,300,400$, $500 \mathrm{~nm}$ ). The CBC value density distributions for mNeonGreen-tagged proteins relative to PAmCherrytagged proteins is shown in green, while the opposite is shown in red. Interval width $=5 \mathrm{~nm}$.

f, The percentage of CBC values above or equal to 0.5 relative to $R$ derived from (a-e) is plotted for each fluorescent protein pair and compared to the positive and the negative control.

$\mathbf{g}, \mathbf{h}, \mathrm{CBC}$ value density distributions calculated for simulations characterized by increasing mNeonGreen concentrations $\left(25,50,100,200,400\right.$ and 800 molecules $\left./ \mu^{2}\right)$ and constant PAmCherry concentration $(\mathbf{g}) . R_{\max }=300 \mathrm{~nm}$, interval width $=10 \mathrm{~nm}$. The percentage of CBC values above or equal to 0.5 (mNeonGreen to PAmCherry and vice versa) was determined and compared for all mNeonGreen densities to evaluate the necessity of separate negative controls for each protein pair $(\mathbf{h})$. 
a

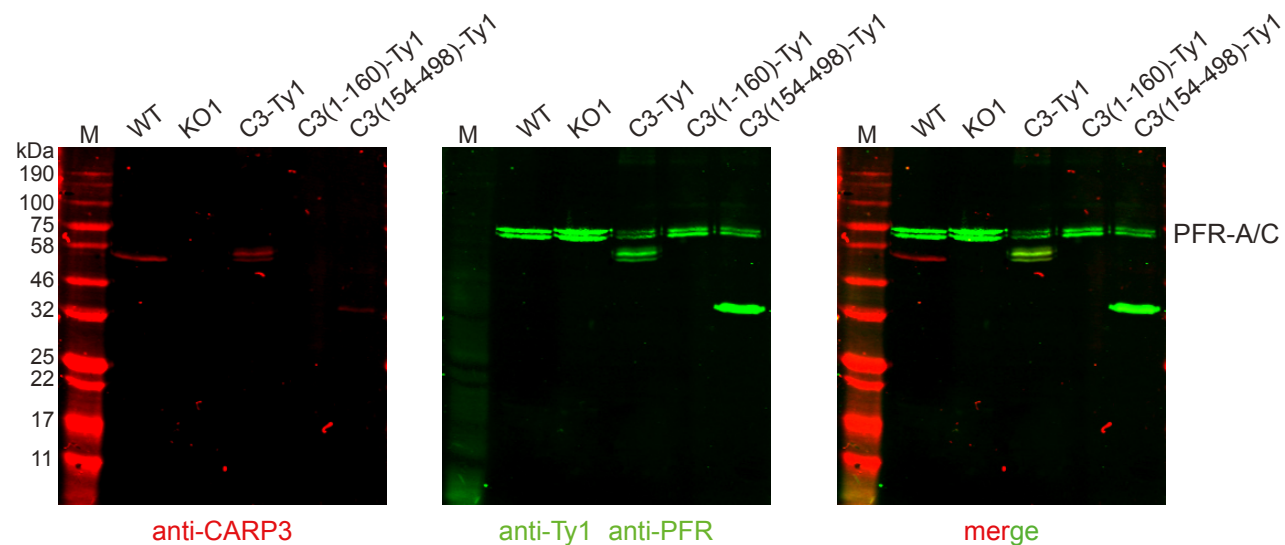

b

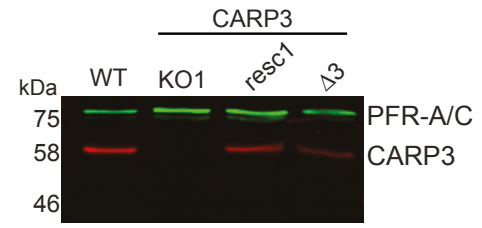

Extended Data Fig. 4 Expression of CARP3 mutant proteins

a, Western blot analysis of procyclic T. brucei AnTat 1.1 constitutively overexpressing CARP3-Ty1, CARP3(1-160)-Ty1 or CARP3(154-498)-Ty1 or b, in situ rescue with full-length CARP3 (resc1) or

$C A R P 3 \triangle 3(\triangle 3)$ in a carp3 knock out (KO) background. Wild type (WT) and KO (KO1) cell lines were included as controls. Western blots were probed with anti-CARP3, anti-Ty1 (only in (a)) and anti-PFRA/C (loading control).

Note that CARP3(1-160)-Ty1 (calculated molecular weight $19.5 \mathrm{kDa}$ ) is not detectable by Western blot but only by immunofluorescence microscopy (see Fig. 2k). M: protein molecular weight marker. 
a
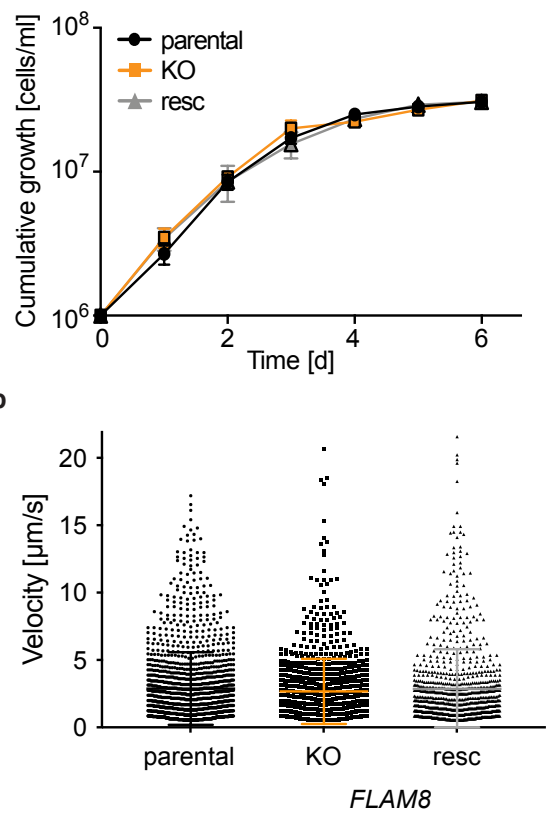

c $\quad$ CARP3-PAmCh

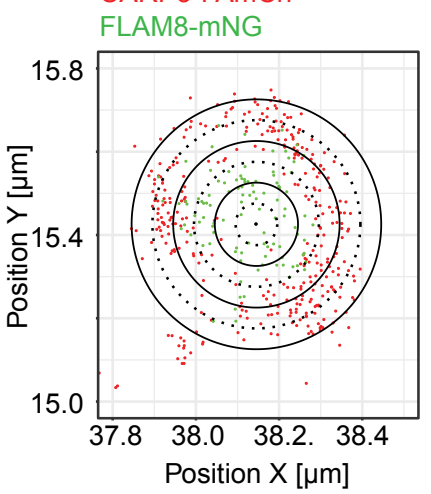

Extended Data Fig. 5 FLAM8 knock out cells have no growth or motility phenotype

$\mathbf{a}, \mathbf{b}$, Representative growth curves (a) and analysis of single cell mean velocity (b) of AnTat 1.1E 'Paris' parental, flam8 knock out (KO) or FLAM8 rescue (resc) expressing the red triple marker. (b) shows mean \pm SD of $n=1502$ (parental); $n=898$ (KO); $n=$ 898 (resc).

c, Single molecule localization of CARP3-PAmCherry and FLAM8-mNG from Fig. 3e shown via centroids. Rings are drawn in $50 \mathrm{~nm}$ steps from center of flagellar tip crosssection. Dotted lines $50 \mathrm{~nm}$, solid lines $100 \mathrm{~nm}$. 


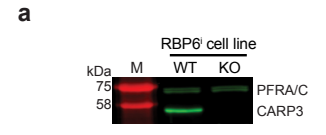

b
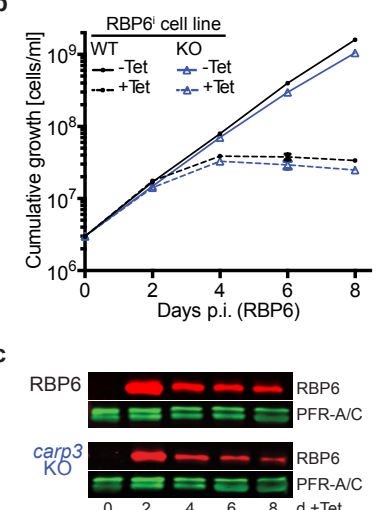

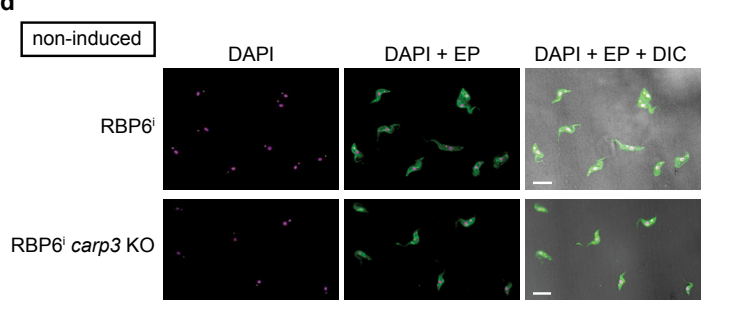

$4 \mathrm{~d}+$ Tet

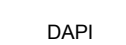

DAPI +EP

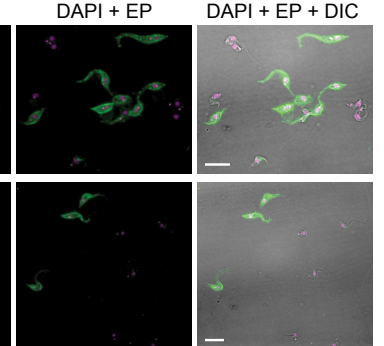

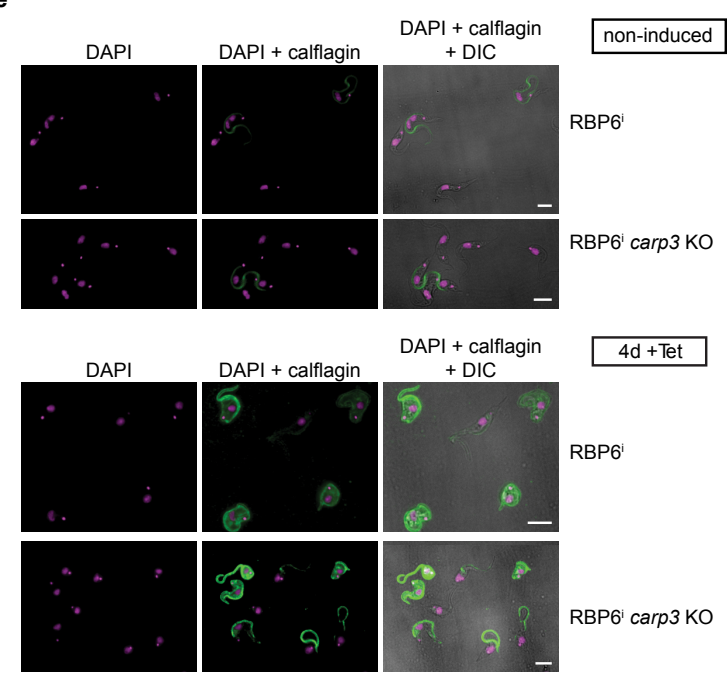

Extended Data Fig. 6 CARP3 KO parasites are fully differentiation-competent in the in vitro RBP6 overexpression system

a, Western blot analysis of CARP3 expression in a homozygous deletion mutant of carp3 (KO) generated in a procyclic T. brucei EATRO 1125 cell line allowing (Tet)-inducible RBP6 overexpression (RBP6i). PFR-A/C serves as loading control.

, c, Cumulative growth (b) and Western blot (c) analyses of cell lines as in (a) with or without Tet induction $(10 \mu \mathrm{g} / \mathrm{mL})$ of RBP6 overexpression. Growth curves are mean \pm SD of $n=3$ for $+T e t ; n=1$ for -Tet. Western blots show inducible overexpression of RBP6 for both cell lines. PFR-A/C

$\mathbf{d}, \mathbf{e}$, Indirect immunofluorescence analysis of EP procyclin (d) or calflagin (e) in the cell lines indicated. Marker protein expression was analyzed before (non-induced; upper panels) and four days after induction of RBP6 expression with $10 \mathrm{\mu g} / \mathrm{mL}$ tetracycline (4d +Tet; lower panels).

DNA was stained with DAPI (magenta). Scale bars $5 \mu \mathrm{m}$. 

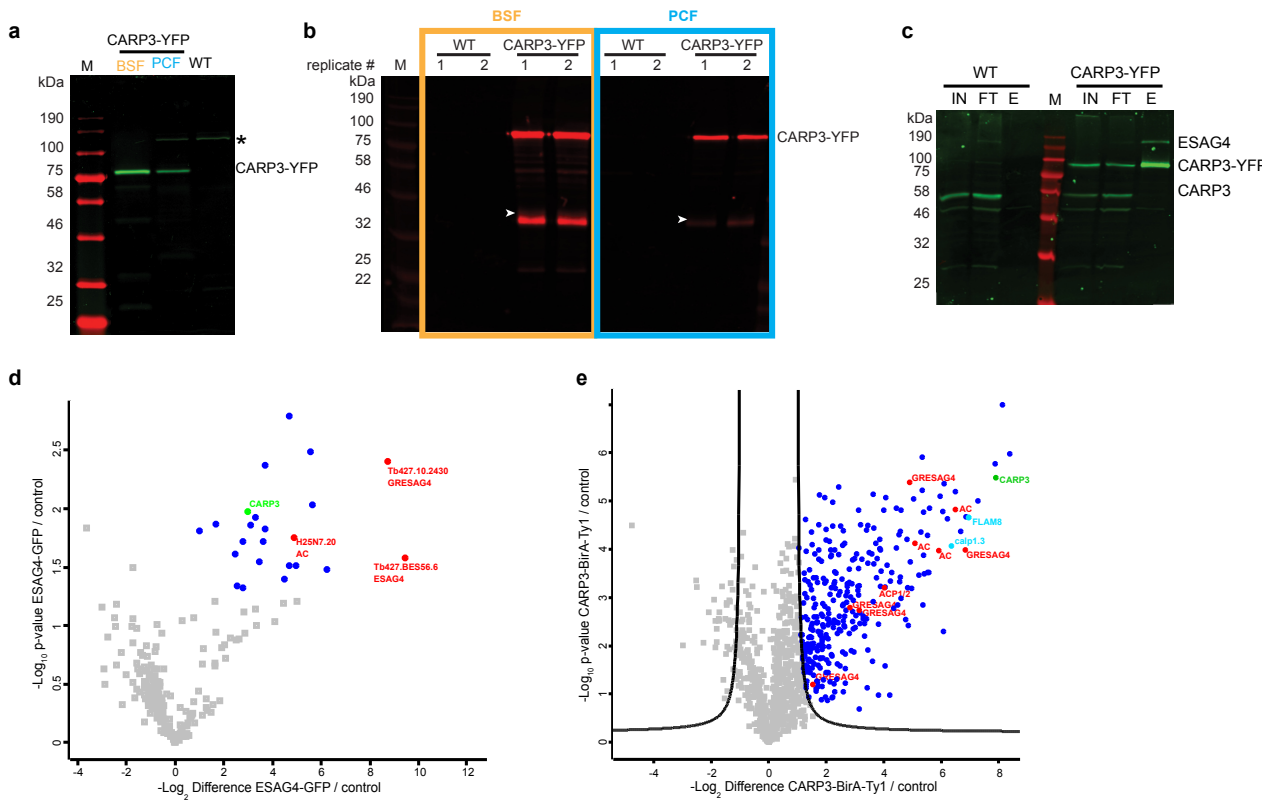

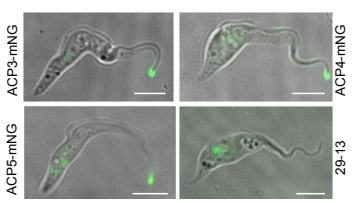

g

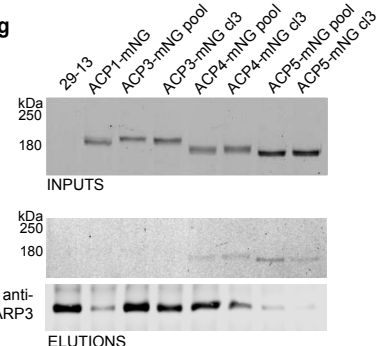

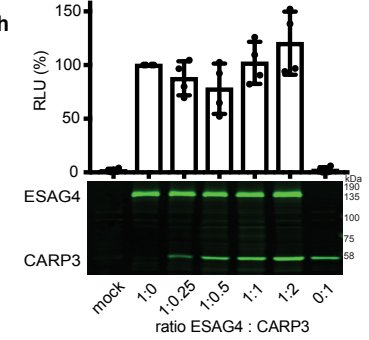

Extended Data Fig. 7 CARP3 interacts with ACs and regulates their abundance

a, In-gel fluorescence of T. brucei BSFs and PCFs of strain AnTat 1.1E expressing CARP3-YFP in situ. Procyclic wild type cells (WT) were loaded as control. The asterisk $\left(^{*}\right)$ marks an autofluorescent fumarate reductase ${ }^{124}$.

b, GFP trap pull-down of cell lines from (a). The Western blot probed with anti-CARP3 shows the eluted fractions of two replicates each. The band at $\sim 35 \mathrm{kDa}$ (white arrowhead) is probably a proteolytic degradation product of CARP3. c, GFP trap pull-down in T. brucei MiTat 1.2 BSF wild type (WT) or CARP3-YFP cells. The Western blot was probed with anti-CARP3 and anti-ESAG4. IN input; FT flow-through; E elution.

d, GFP trap pull-down in T. brucei MiTat 1.2 BSF 13-90 (control) or derived ESAG4-GFP expressing cells. The in-ge fluorescence analysis shows expression and solubility of ESAG4-GFP upon detergent lysis in the input fraction and pull-down of ESAG4-GFP in the eluted fraction. The Volcano plot displays proteins plotted according to $p$-value and fold change derived from mass spectrometry analysis of two replicate pull-downs. Significantly enriched proteins

(ESAG4-GFP / control) ( $p$-value $\leq 0.05$ ) are represented by blue dots. AC isoforms are shown in red, CARP3 in green. e, CARP3 proximity proteomics using BioID. Volcano plot representation of CARP3 BiolD comparing pull-down of biotinylated proteins from BSF T. brucei $\Delta$ carp3/CARP3-BirA*-Ty1 versus $\Delta$ carp3/CARP3. Proteins are plotted according to $p$-value and fold change. Significantly enriched proteins (CARP3-BirA*-Ty1 / control) ( $p$-value $\leq 0.05$, $s_{0}=2$ ) are represented by blue dots and localize above the significance line on the right. CARP3: green; FLAM8 and calpain 1.3: cyan; AC isoforms: red.

f, Fluorescence microscopy of procyclic T. brucei 29-13 and derived ACP3-, ACP4- or ACP5-mNeonGreen expressing cells. Scale bars $5 \mu \mathrm{m}$.

g, CARP3 IP in procyclic T. brucei 29-13 and derived ACP1-, ACP3-, ACP4- or ACP5-mNeonGreen expressing cell lines. Upper panel: in-gel fluorescence analysis of ACP-mNG in input fractions (INPUTS). Lower panels: in-gel fluorescence analysis of ACP-mNG and Western blot detection of CARP3 in eluted fractions (ELUTIONS).

h, Dual-Luciferase® cAMP reporter assay in HEK cells transfected with ESAG4 and CARP3 expression vectors at the indicated ratios. Equal amounts of total DNA were transfected in all conditions. Relative light units (RLU) of the dualluciferase assay were normalized to ESAG4 protein levels with one representative Western blot (anti-CARP3, antiESAG4) shown. The RLU value for the 1:0 ratio of ESAG4:CARP3 was set to $100 \%$. The graph shows mean \pm SD of four independent biological replicates. 


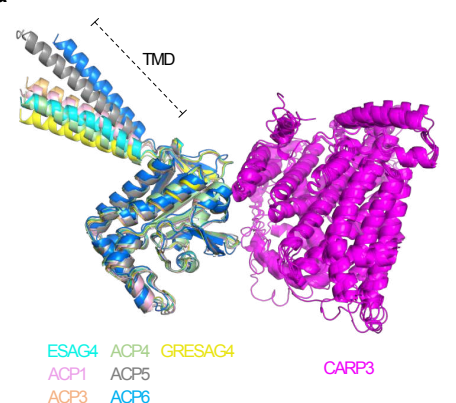

b

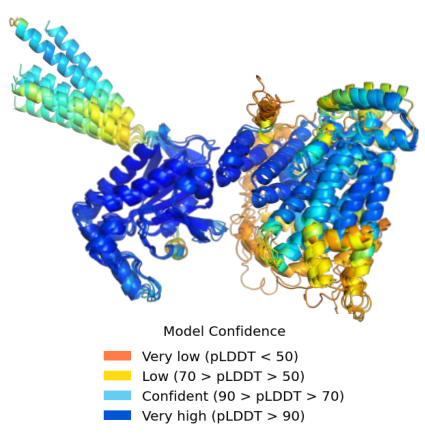

d
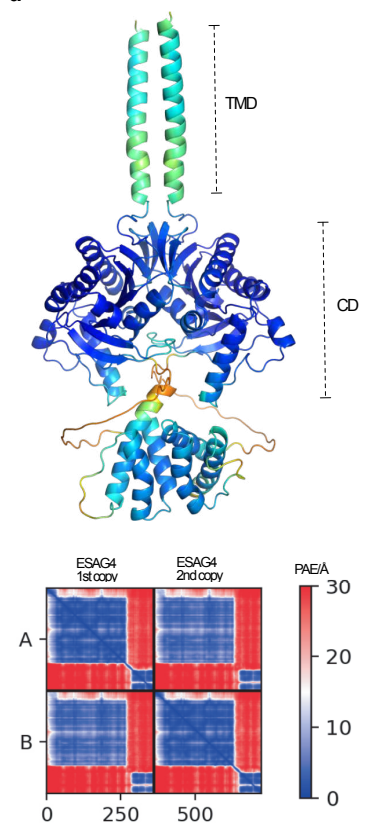
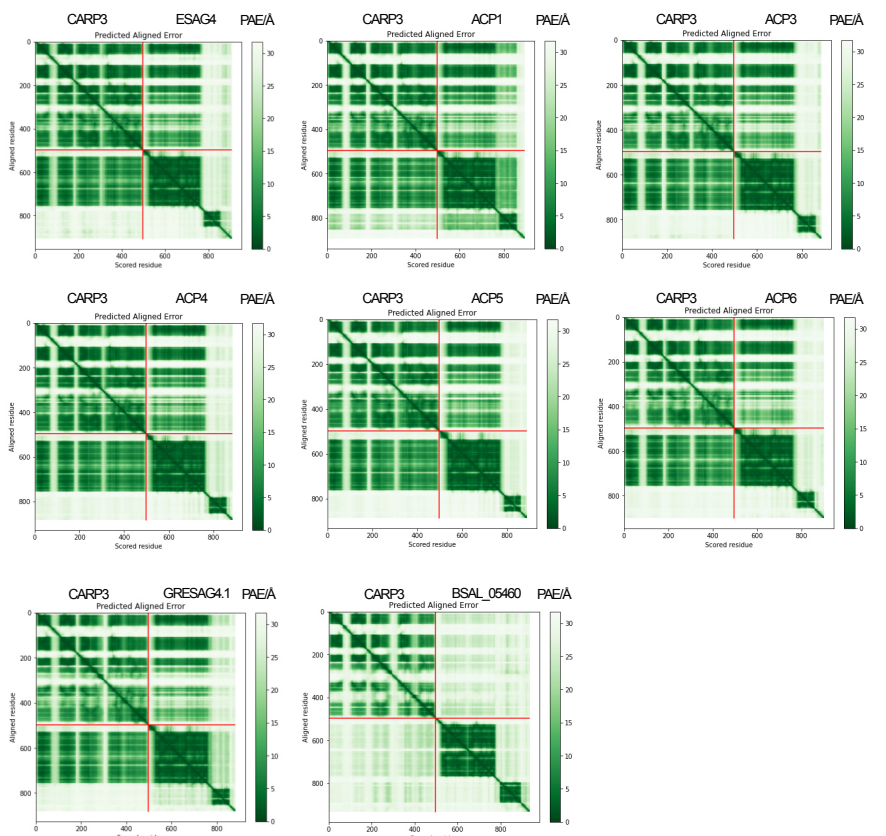

Extended Data Fig. 8 Structure modeling of CARP3-AC complexes using AlphaFold

a, Cartoon representation of seven AlphaFold-generated models of CARP3 (magenta) in complex with the intracellular catalytic domain of different $A C$ s (each $A C$ isoform is shown by a different color, as indicated; ESAG4: Tb427.BES40.13; ACP1: Tb927.11.17040; ACP3: Tb927.7.7470;ACP4: Tb927.10.13040; ACP5: Tb927.11.13740; ACP6: Tb927.9.15660; GRESAG4.1: Tb927.6.760). The transmembrane domain (TMD) of the receptor-type ACs is labeled. The AC extracellular N-terminal part was not included in the structure prediction. The models are shown after superpositioning of CARP3.

b. Same as panel (a) but colored according to local model confidence using the predicted local-distance difference test (pLDDT) score.

c, Predicted alignment error (PAE) plots for the models shown in panels $(a, b)$, indicating the confidence of the complex modelling. The PAE for the predicted model of $T$. brucei CARP3 in complex with a receptor-type transmembrane AC (BSAL_05460) from the distantly related kinetoplastid Bodo saltans that lacks a CARP3 orthologue was included as a negative control.

d, AlphaFold-generated model for an ESAG4 AC homo-dimer (ESAG4 amino acids 862-end, lacking the extracellular $\mathrm{N}$-terminal part) color-coded according to pLDDT score. TMD transmembrane domain; CD catalytic domain. 
1822 Supplementary Fig. 1 Expression or localization of CARP3 is not dependent on intracellular cAMP levels

a, Pull-down assay using cAMP-coupled agarose beads (2-AHA or 8-AHA linker) and lysate of T. brucei AnTat 1.1E expressing PDEB1-mNeonGreen (PDEB1-mNG). PDEB1- mNG serves as positive control for cAMP binding

1827 and was detected by in-gel fluorescence; CARP3 was detected by

1828 immunoblotting using rabbit anti-CARP3. Lanes represent input material (I), 1829 flow-through (FT), washes (W) and eluted material (E).

1830 b, Fluorescence microscopy of CARP3-YFP (green) in procyclic T. brucei 1831 AnTat 1.1E in the presence $(24 \mathrm{~h}, 48 \mathrm{~h}$ ) or absence (control) of $1 \mu \mathrm{M} \mathrm{CpdA}$ or 1832 CpdB for $24 \mathrm{~h}$ or $48 \mathrm{~h}$, respectively. Scale bars $5 \mu \mathrm{m}$.

1833 c, Social motility assay of CpdA- or CpdB-treated $(1 \mu \mathrm{M})$ or untreated cells as 1834 in (b).

1836 Supplementary Table 1. Summary of coordinate-based colocalization 1837 (CBC) analysis.

1838

1839 Supplementary Table 2. CARP3-YFP pull-down in bloodstream forms 1840 (2a) or procyclic forms (2b).

1842 Supplementary Table 3. ESAG4-GFP pull-down in bloodstream forms. 1843 
1844 Supplementary Table 4: CARP3 BiolD proximity proteomics identifies 1845 putative CARP3 interactors.

1846

1847 Supplementary Table 5. Quantitative proteomics upon CARP3 knock 1848 down.

1849

1850 Supplementary Table 6: Summary of AC isoforms identified by proteome 1851 studies in Supplementary Tables 2, 4 and 5.

1852

1853 Supplementary Table 7: List of Primers used for cloning. 1854 


\section{Supplementary Files}

This is a list of supplementary files associated with this preprint. Click to download.

- SuppTable1.xlsx

- SuppTable2.xlsx

- SuppTable3.xlsx

- SuppTable4.xlsx

- SuppTable5.xlsx

- SuppTable6.xlsx

- SuppTable7.xlsx

- SupplementaryFig1.pdf

- PDBExtendedDataFig.8a.txt

- PDBExtendedDataFig.8d.txt

- pymolExtendedDataFig.8a.pse

- pymolExtendedDataFig.8d.pse 